\title{
Advances in Biosensor Technologies for Acute Kidney Injury
}

\author{
Esma Derin and Fatih Inci*
}

Cite This: ACS Sens. 2022, 7, 358-385

Read Online

\begin{abstract}
ACCESS I
山 Metrics \& More

ABSTRACT: Acute kidney injury (AKI) is one of the most prevalent and complex clinical syndromes with high morbidity and mortality. The traditional diagnosis parameters are insufficient regarding specificity and sensitivity, and therefore, novel biomarkers and their facile and rapid applications are being sought to improve the diagnostic procedures. The biosensors, which are employed on the basis of electrochemistry, plasmonics, molecular probes, and nanoparticles, are the prominent ways of developing point-of-care devices, along with the mutual integration of efficient surface chemistry strategies. In this manner, biosensing platforms hold pivotal significance in detecting and quantifying novel AKI biomarkers to improve diagnostic interventions, potentially accelerating clinical management to control the injury in a timely manner. In this review, novel diagnostic platforms and their manufacturing processes are presented comprehensively. Furthermore, strategies to boost their effectiveness are also indicated with several applications. To maximize these efforts, we also review various biosensing approaches with a number of biorecognition elements (e.g., antibodies, aptamers, and molecular imprinting molecules), as well as benchmark their features such as robustness, stability, and specificity of these platforms.
\end{abstract}

Article Recommendations

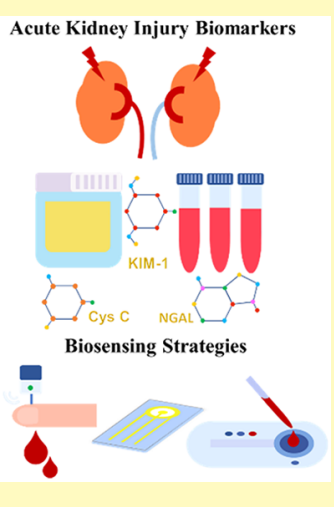
KEYWORDS: Acute kidney injury (AKI), AKI biomarkers, early diagnosis, point-of care (POC), biosensors, electrochemical biosensors, plasmonic biosensor, nanoparticles, molecular probes, commercial tests

A cute kidney injury (AKI), also known as acute renal failure (ARF), has an enormous impact at an alarming rate, leading to the death of $\sim 2$ million people annually across the globe. ${ }^{1}$ The incidence rate of as much as one in five hospitalized patients accounts for more than 13 million individuals every year, making AKI one of the primary concerns in public health. ${ }^{2,3}$ Regrettably, the mortality and morbidity caused by AKI are rising enormously in both clinics and intensive care units (ICUs). ${ }^{4}$ Besides the mortality and morbidity, the economic burden of AKI is noteworthy, since a hospitalized patient who developed AKI needs to pay an additional USD 9000 compared to a hospitalized patient who did not develop AKI. Furthermore, the development of AKI is highly associated with the risk of more extended or unplanned ICU stays, close monitoring of a patient, and prolonged hospitalization or early rehospitalization. ${ }^{5}$

AKI is a severe renal disease comprising an abrupt decrease in kidney function within 7 days or less in accordance with The Kidney Disease: Improving Global Outcomes (KDIGO), the most updated consensus for AKI that creates a network to establish the standards and criteria for AKI diagnosis. ${ }^{1,6}$ Basically, kidney function is assessed by monitoring solutes that are supposed to be cleared under normal conditions by the kidney and urine volume by considering volume status. ${ }^{6}$ Currently, diagnostic criteria employ the Acute Kidney Injury Network (AKIN) classification, RIFLE, which represents the risk (dysfunction), injury, failure, loss (kidney function), and end-stage kidney disease, or KDIGO definitions. ${ }^{1,2,7-9}$ The diagnostic criteria are serum creatinine $(\mathrm{SrC})$, blood urea nitrogen (BUN), and urine output (UO). ${ }^{10,11} \mathrm{SrC}$ and UO are utilized to estimate or evaluate the glomerular filtration rate (GFR), which also reflects AKI. ${ }^{12}$ Both $\mathrm{SrC}$ and urine output are still widely used as conventional diagnoses, yet they are not sensitive and specific enough for early determination of AKI, because the elevation of SrC levels occurs 24-36 h after the injury. $^{12}$

The etiologies of AKI are investigated in three categories, (i) pre-renal (e.g., volume depletion, systemic hypotension, significant renal vascular stenosis or thrombosis, severe systolic or diastolic cardiac failure, and activation of the neurohumoral axis increasing renal vascular resistance), (ii) renal (including direct intrinsic kidney damage such as glomerulopathies, acute vasculitis, acute interstitial nephritis, and acute tubular necrosis), and (iii) post-renal (e.g., the obstruction of the urinary tract). ${ }^{13}$ Based on the traditionally measured parameters, the several etiologies of AKI cause different outcomes and effects. The inefficiency of these traditional parameters has led researchers to seek alternative molecules that are released from injured kidneys to indicate the abnormality, as well as being easy to collect from bodily fluids. ${ }^{14}$ These molecules cannot be only constitutive proteins;

Received: August 19, 2021

Accepted: November 18, 2021

Published: December 20, 2021 
they might also be elevated molecules (e.g., proteins, encapsulated molecules in exosomes, miRNA, and siRNA) as a response to an injury. ${ }^{11}$ The possibility of the presence of sensitive molecules and specific markers for AKI in urine is promising for the development of diagnostic tools. ${ }^{15}$ Therefore, novel diagnostic tools along with proper biomarkers have been required to eliminate these disadvantages and potentially provide a noninvasive strategy for early diagnosis. ${ }^{16}$ To address this requirement, researchers have introduced various novel AKI biomarkers such as $N$-acetyl- $\beta$-D-glucosaminidase (NAG), neutrophil gelatinase-associated lipocalin (NGAL), kidney injury molecule-1 (KIM-1) and interleukin-18 (IL-18), cystatin $\mathrm{C}$ (Cys $\mathrm{C}$ ), $\beta$-2-microglobulin, liver fatty acid-binding protein (L-FABP), tissue inhibitor of metalloproteinases-2/insulin-like growth factor-binding protein 7 (IGFBP7/TIMP-2), and microRNAs. ${ }^{15,17-20}$

Aforementioned novel urinary biomarkers are classified based on different approaches: for instance, low molecular weight (freely filtered), elevated levels in kidney cells, and expressed biomolecules in tubular epithelial cells that are released to urine. ${ }^{17}$ Another classification strategy can be based on functionality and structure, such as tubular enzymes and upregulated proteins. $\beta 2$-Microglobulin, lysozyme, $\alpha 1$-microglobulin, light chains, and cystatin $C$ are low-molecular-weight proteins that are capable of being easily filtered in the glomerulus and reabsorbed in the proximal tubules. NGAL and KIM-1 are, on the other hand, produced in tubular epithelial cells after an injury. The hepatocyte growth factor (HGF) level also increases in interstitial cells due to an injury in the kidney. Lysosomal enzymes such as NAG, cytosolic protein lactate dehydrogenase, and gp130 are biomarkers, which are released into urine. ${ }^{17}$

Elaborating more on the biomarkers, NAG is a $140 \mathrm{kDa}$ hydrolytic lysosomal enzyme that originates from the lysosome of a proximal tubule cell to form structural components in tissues by breaking bonds between glycosides and amino sugars. ${ }^{21}$ It holds potential as a sufficient biomarker due to an increase in urine as a result of proximal tubule injuries, leading to the loss of lysosome integrity. ${ }^{22}$ When the tubular cell injury occurs, the activity of this enzyme increases to reflect tubular cell function. NAG cannot be filtered through the glomerulus by the virtue of its high molecular weight. During an active kidney disease, urinary NAG level increases perpetually. Toxic agents, post-cardiac surgery or post-renal transplantation are the central inductions of the elevated levels of urinary NAG. ${ }^{23}$ Mainly, the increase of NAG activity reflects proximal tubular cell injury; however, lysosomal activity or leukocyte degranulation can be demonstrated by high concentrations of NAG without tubular damage. ${ }^{21}$

Neutrophil gelatinase-associated lipocalin (NGAL) (25 $\mathrm{kDa})$, as known as lipocalin-2, 24p3, siderocalin or uterocalin, is mostly studied renal injury biomarker for AKI by the virtue of its rapid response to tubular damage. NGAL can be filtered easily and reabsorbed in the glomerulus, and then, in the proximal tubule. ${ }^{24,25}$ It is associated with the mechanisms for bacterial defense, and also responsible for neutrophil maturation, signal transduction, and major renal tubular damage. $^{25}$ Under pathological conditions including epithelial cell damage, inflammation or bacterial infection, NGAL is excreted from mostly neutrophils, epithelial cells, kidney tubular cells, and hepatocytes as a response to tubular damage since it can be described as a biomarker of acute renal lesions. The levels of NGAL are quantified from serum, plasma, and urine; however, the specificity of serum is lower in comparison to urine. ${ }^{24-26}$ When the injury occurs in proximal tubular epithelial cells, systemic and urinary NGAL concentrations are elevated as it is excreted by the loop of Henle and collected by ducts. $^{17,25}$

Kidney injury molecule-1 (KIM-1) is a type 1 membrane protein $(38.7 \mathrm{kDa})$ during $\mathrm{AKI}$, and its level is significantly low or even absent in healthy individuals. Type 1 membrane protein-a membrane receptor, contains $\mathrm{T}$ cell immunoglobulin and mucin domain-containing 4 (TIMD4) in the extracellular part. ${ }^{7,17,27,28} \mathrm{KIM}-1$ has a substantial role in the immune system, and several studies have indicated that the control of viral infections, autoimmunity, immune tolerance, and atopic diseases are associated with AKI. Varying immune dysfunction is also associated with KIM-1 such as allergies, asthma, ectopic dermatitis, rheumatoid arthritis, and systemic lupus erythematosus. ${ }^{28}$ When an injury occurs, KIM-1 levels increase in both urine and kidney within a short time; therefore, it can be a reporting molecule for an early diagnosis of the kidney injury. In addition, this elevation is associated with the severity of the condition. ${ }^{28}$ While the regeneration is taking place in epithelial cells at proximal tubular, the expression of mRNA and proteins of KIM-1 rises, and it primarily due to ischemia, nephrotoxicity, hypoxia, some renal tubular interstitial, and polycystic kidney disease. ${ }^{27-30}$ KIM-1 can be used for cisplatin-induced and ischemic AKI in rats as a urinary biomarker due to its noninvasive, sensitive, rapid, and reproducible fashions. It can also be used as a tissue-based and urinary biomarker for nephrotoxicity-induced kidney injury. ${ }^{7}$

IL-18 is a proinflammatory cytokine from the IL- 1 family, and the molecular weight of IL-18 is $23-24 \mathrm{kDa}$ before its activation with cysteine protease caspase-1, and then, the molecular weight decreases to $18.3-17 \mathrm{kDa}$ due to the cleavage of precursor. ${ }^{31-33}$ IL-18 is associated with the ischemic proximal tubular injury in mice and proinflammatory responses. The mechanism starts with production in the proximal tubules, and then, it is activated by caspase- 1 . The activated IL-18 is secreted to urine; therefore, it can be considered as a biomarker for AKI. ${ }^{31}$ Recent studies have revealed that renal ischemia-reperfusion injury could be the induction of acute tubular necrosis and neutrophil and monocyte infiltration of the renal parenchyma. IL-18 level is elevated in comparison to healthy individuals when the ATN, urinary tract infection, chronic renal insufficiency, and prerenal azotemia are present. ${ }^{33}$

Cys C, another important biomarker, is a $13.359 \mathrm{kDa}$ lowmolecular-weight protein that can be easily filtered in the glomerulus and reabsorbed by the proximal tubules, and it is not secreted to renal tubules again.,29,34 Cys $C$ has negative correlation to the GFR levels. When an injury exists in kidney, serum Cys $\mathrm{C}$ increases along with the other low-molecularweight proteins. ${ }^{34}$ The significant increase in Cys C levels reflects proximal tubular injury and impairment of reabsorption. 32

\section{BIOSENSING STRATEGIES FOR AKI DIAGNOSIS}

Biomolecular information is the basis of modern medicine to diagnose and monitor disease conditions and progression. They are not only used for the diagnosis, but also they provide information on the efficacy of drugs and other treatments. Traditionally, standard clinical techniques (e.g., ELISA, chromatography, and mass spectrometry) are performed to obtain this information from bodily fluids with expensive 

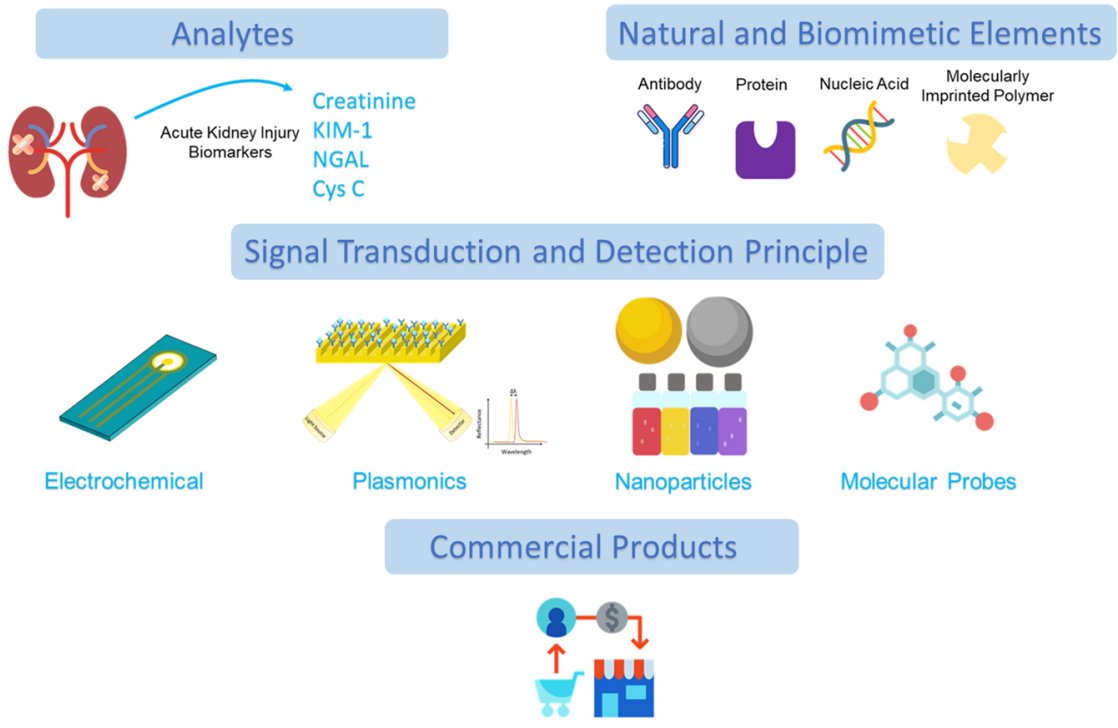

Figure 1. Schematic diagram for acute kidney injury, the associated biosensing strategies, and commercial products. (Figure credits: Some icons used on this figure are obtained from FlatIcon, and they are adapted to the concept accordingly.)

equipment, sophisticated facilities, and time-consuming procedures. In contrast to their drawbacks, some of the clinical techniques are considered as gold standard since they exploit highly reliable and precise results. In addition, assay time should be considered as another critical parameter to improve these listed drawbacks. ${ }^{35}$ Therefore, some alternative strategies providing sensitive, reliable, and cost-efficient analytical measurements (e.g., biosensors and point-of-care (POC) diagnostics tools) should be explored to monitor biomolecules, drugs, and metabolites in clinical samples such as bodily fluids for diagnosis and treatment monitoring. ${ }^{35,36}$ Due to the aforementioned critical reasons, the development of novel, rapid, easy-to-use, cost-effective, sensitive, and specific platforms is urgently needed for early detection and identification of AKI. One of the most common approaches is developing a biosensing platform focusing on detecting novel AKI biomarkers, improving the current practice in the clinics. Various biosensing approaches, such as plasmonic and electrochemical biosensors, can be utilized for that purpose. In addition to these systems, for instance, the integration of nanoparticles is also employed to enhance the diagnostic capability of the sensors. Moreover, several kinds of biorecognition elements (e.g., antibodies, ${ }^{37,38}$ aptamers, ${ }^{39,40}$ molecular imprinting molecules ${ }^{41,42}$ ) are also introduced to increase the robustness and stability of these platforms. The role of biorecognition elements (e.g., enzyme, whole-cell, aptamer, nucleic acids, antibodies, molecularly imprinted polymers, and so on) in biosensing strategies is unique and significant; therefore, selecting the appropriate element would improve the sensitivity and specificity characteristics, which are the two most vital parameters in developing a reliable strategy. ${ }^{43}$ For instance, enzymes have been mostly employed in electrochemical biosensors due to the redox reactions between enzymes and target analytes, and such biosensors utilize the conversion of this information to measurable signals. Specificity of enzymes comes from their binding cavities in 3D structure, catalytic activities in this vicinity, and thereby, targets can interact with enzymes via hydrogen bonding, electrostatics forces, and other noncovalent interactions. ${ }^{44}$ On the other hand, aptamers - single-stranded oligonucleotides, are another type of biorecognition element that are designed via a combinatorial selection process called Systemic Evolution of Ligands by Exponential Enrichment (SELEX). This method is costly, and it requires multiple iterations using a large library of oligonucleotides, thereby requiring more sophisticated facilities. ${ }^{44}$ Antibodies are the most commonly employed bioreceptors to enable sensor surface targeting to the analytes through molecular affinity, and they enable unique recognition patterns with high specificity and accuracy. However, they need multistep protocols to be purified to a level that can provide high specificity, and therefore, their production is not cost-effective. Moreover, they are not so stable under varying temperature and humidity, thereby tending to lose their binding ability. Another drawback of the antibodies is crossreactivity with other antibodies or peptide structures, as well as biological contents such as fibrin and serum proteins that can mask antibody surfaces, hence impeding their interactions with analytes, and eventually reducing the sensitivity and specificity of biosensors. ${ }^{44,45}$ Among these elements, molecularly imprinted polymers (MIPs) have recently introduced an alternative strategy as a new class of biomimetic recognition elements. They are rationally designed to replicate the surface topography of the target molecules on a polymer matrix. In addition, they offer crucial benefits, including high physical stability, facile preparation, robustness, low-cost, and longer shelf life in comparison to antibodies. ${ }^{46}$ The following section introduces detection systems mostly utilizing novel biosensor principles (e.g., electrochemical and plasmonic sensing) in order to determine AKI biomarkers, which is illustrated in Figure 1.

\section{ELECTROCHEMICAL-BASED BIOSENSOR SYSTEMS}

Electrochemical biosensors are a subcategory of the biosensors that utilize biochemical reactions to associate target analytes and generated electrical signals. ${ }^{47}$ The general description of the electrochemical biosensor can be introduced as an analytical device that contains an electrical transducer to convert biochemical events (e.g., enzyme-substrate reaction or antigen-antibody interaction) to electrical signals such as current, voltage or impedance. ${ }^{48}$ For the biochemical response, 

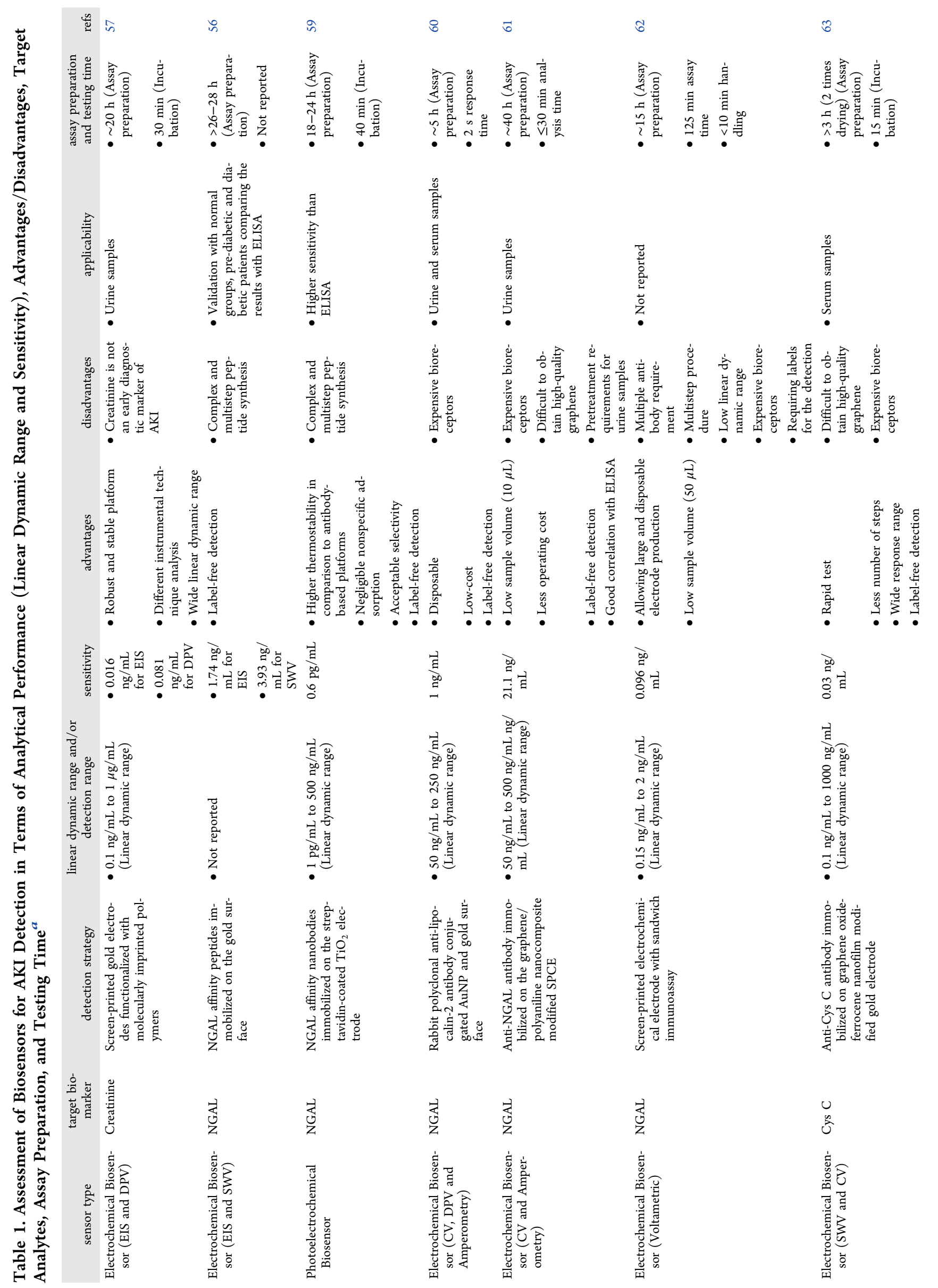

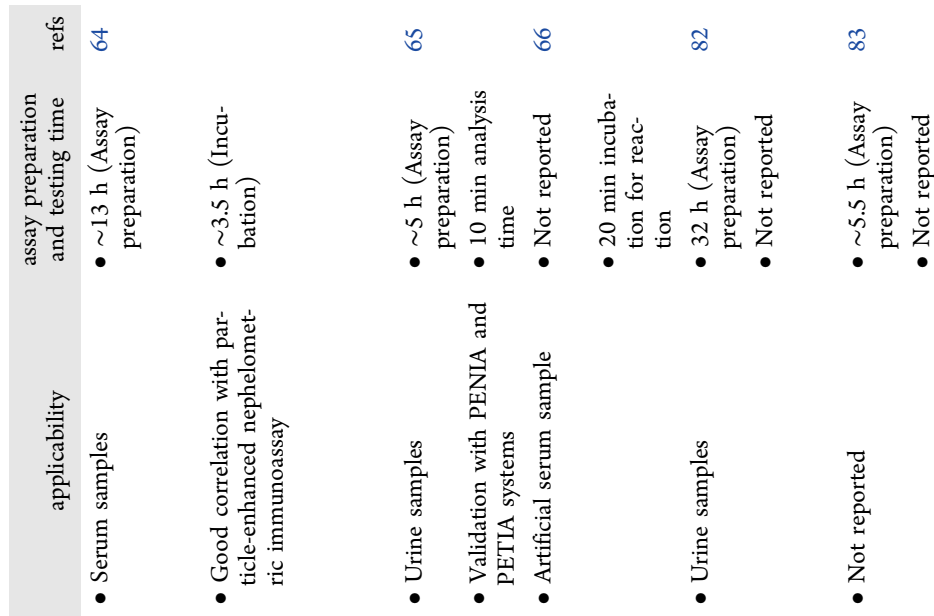

Winuly
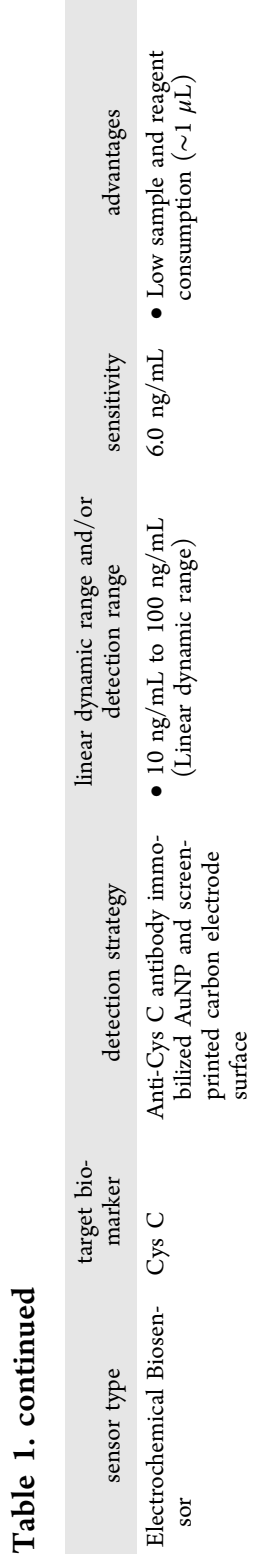

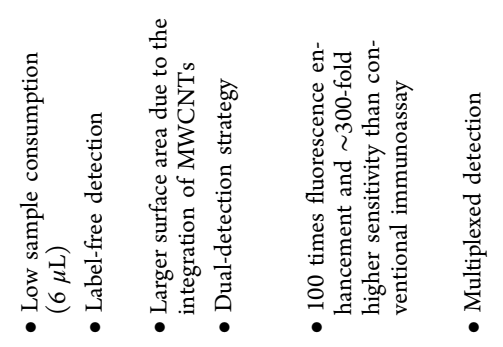

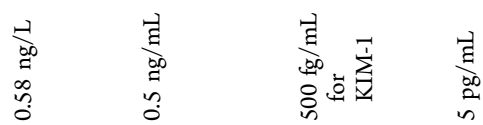
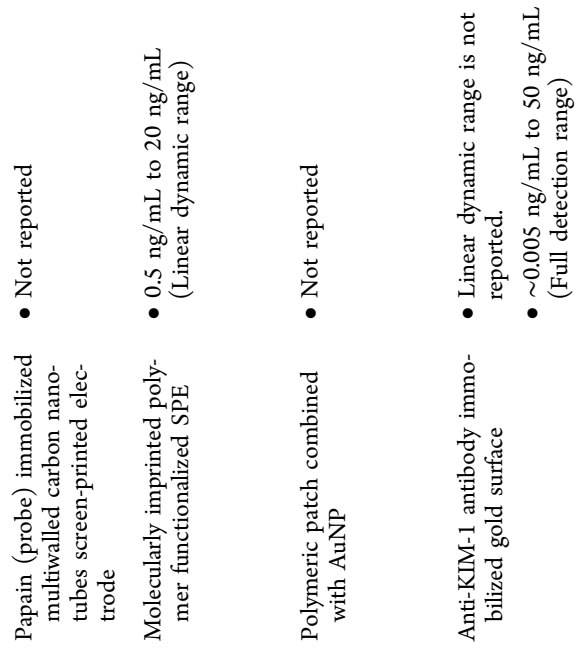

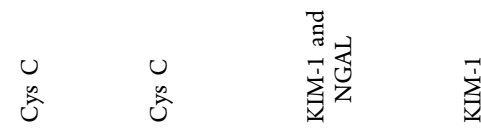

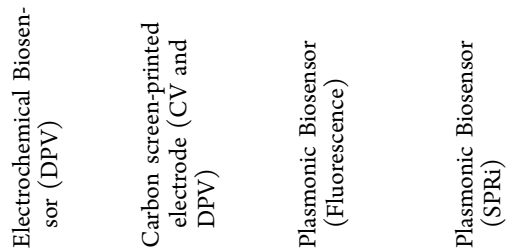

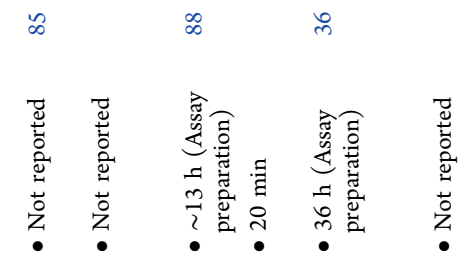

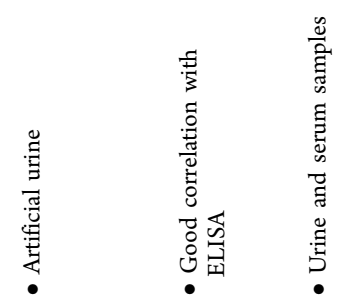




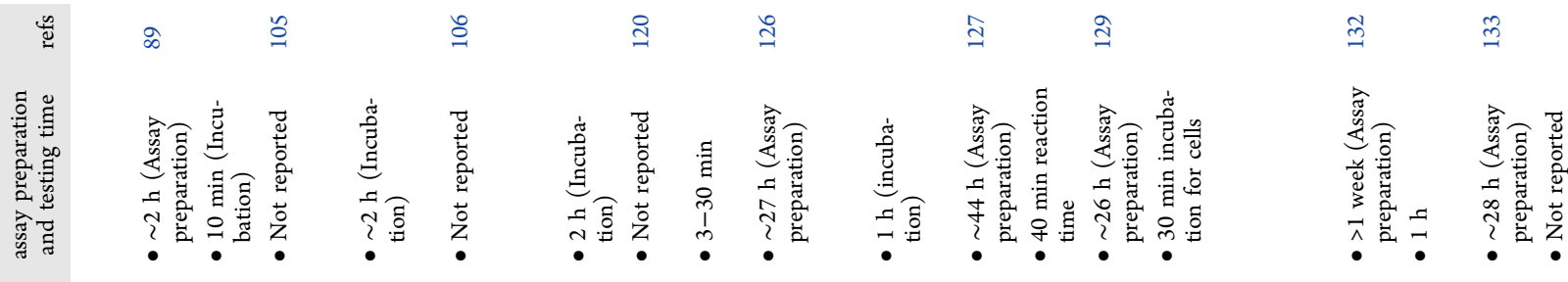

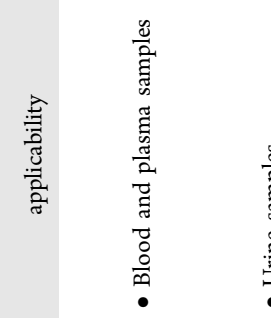
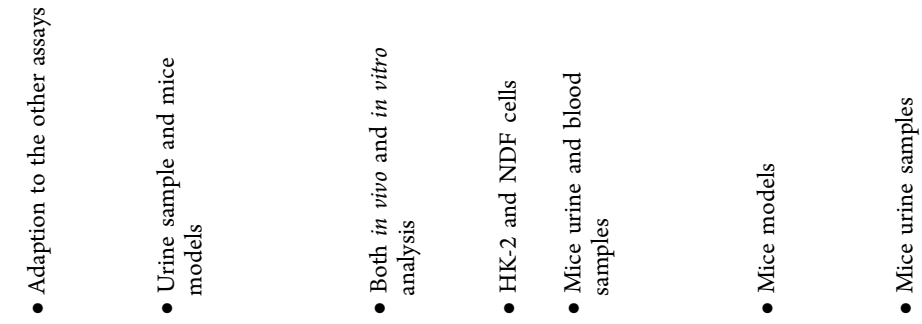

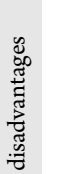

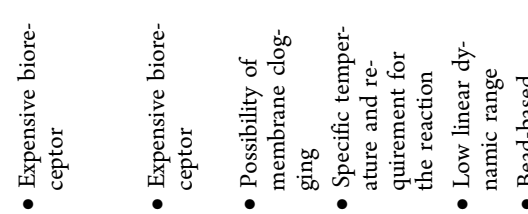

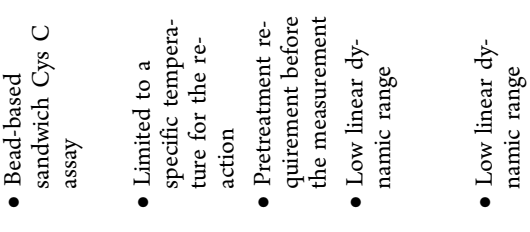

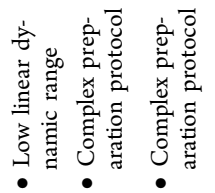
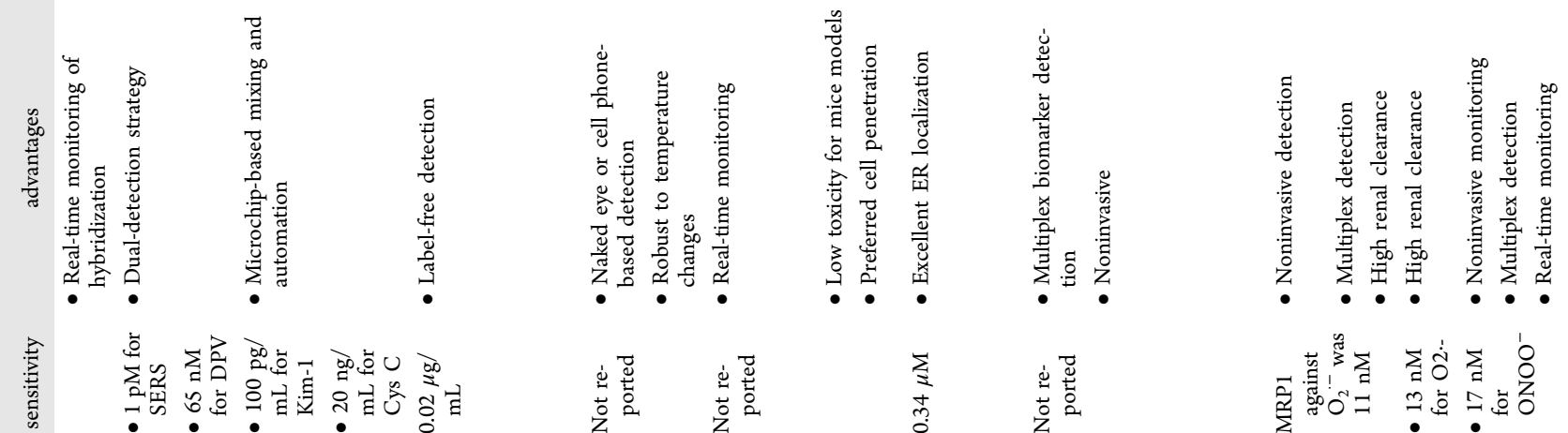

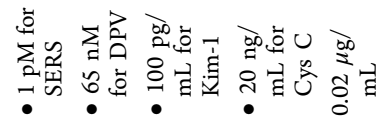

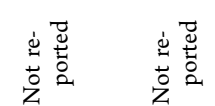

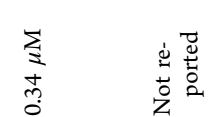

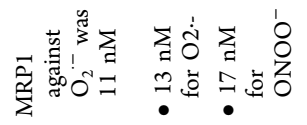
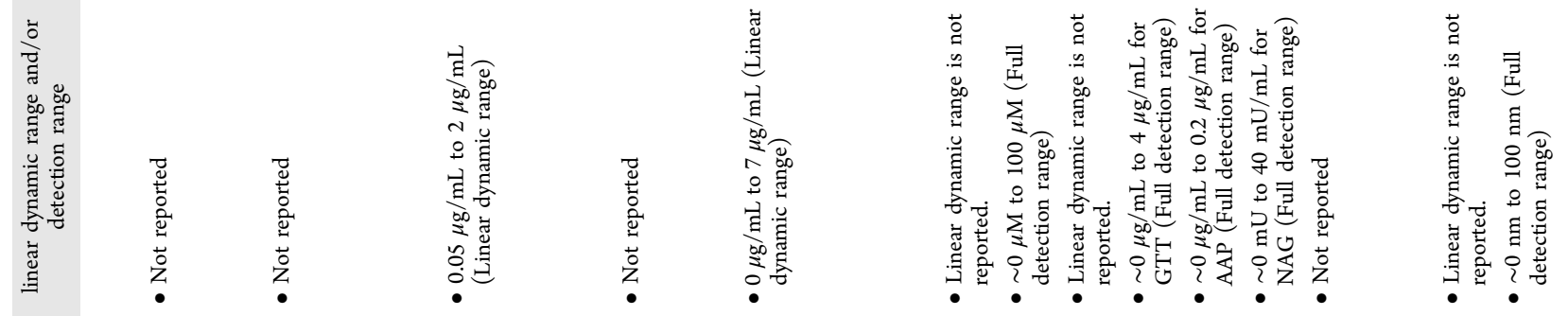

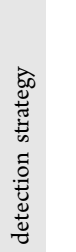
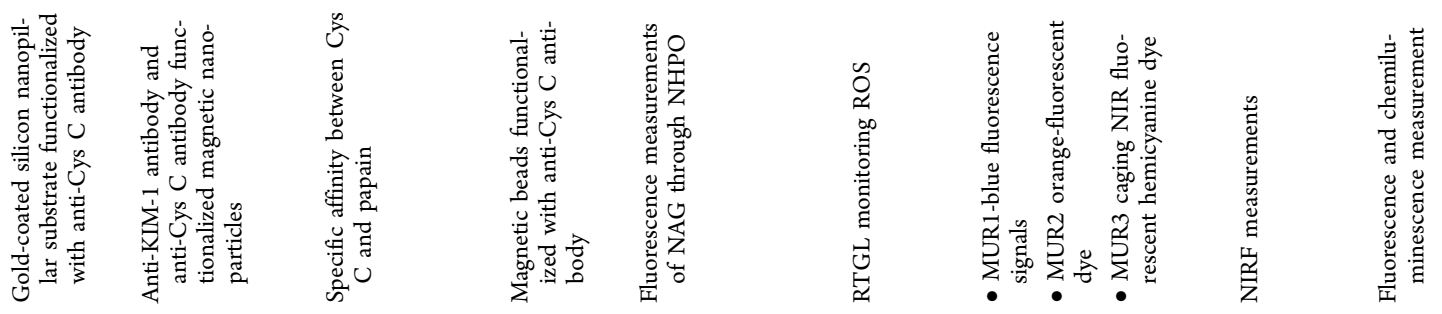

总竞

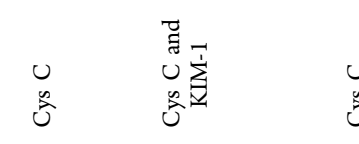

$\begin{array}{lll}0 & 0 & 0 \\ 0 & 0 & 0 \\ 0 & 0 & 0\end{array}$

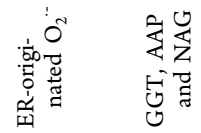

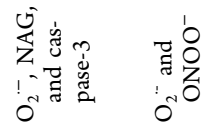
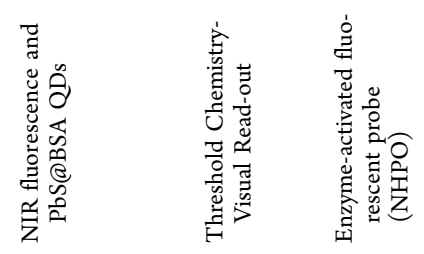

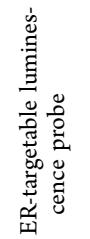

$\sum_{\text {है }}^{2}$

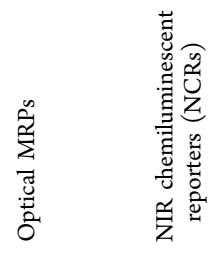




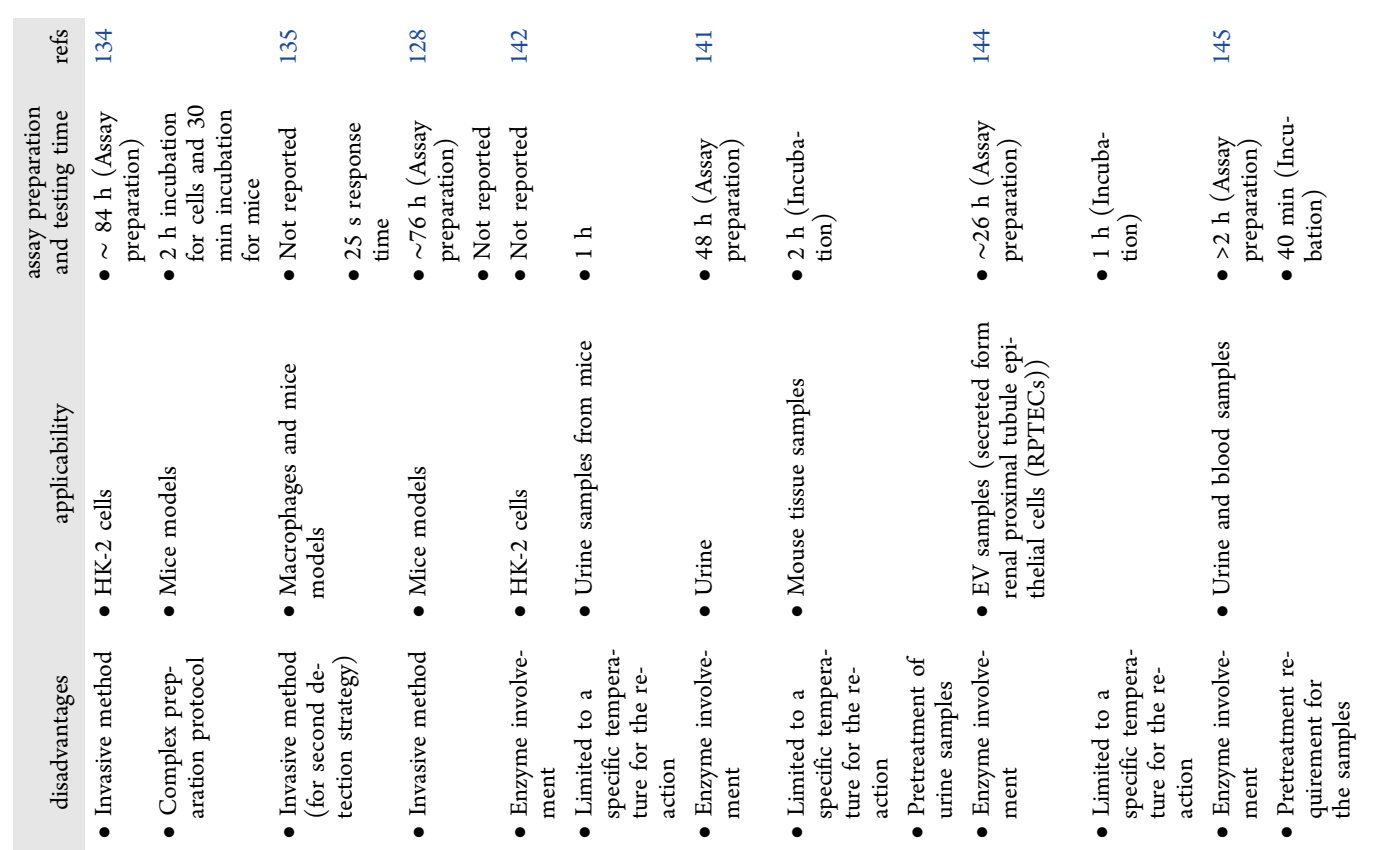

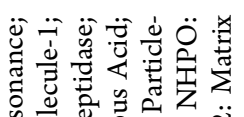

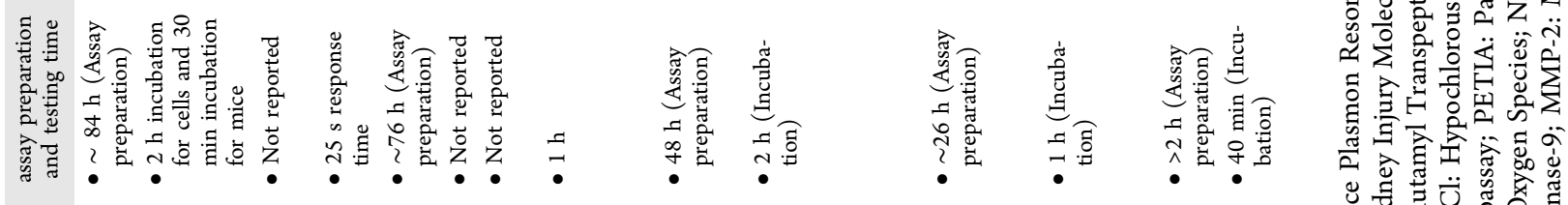

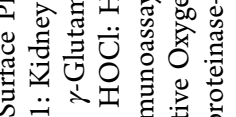

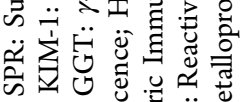

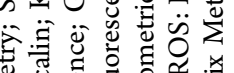

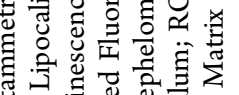

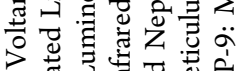

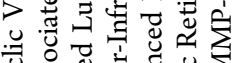

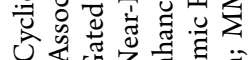
药 U.

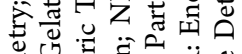

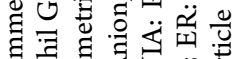

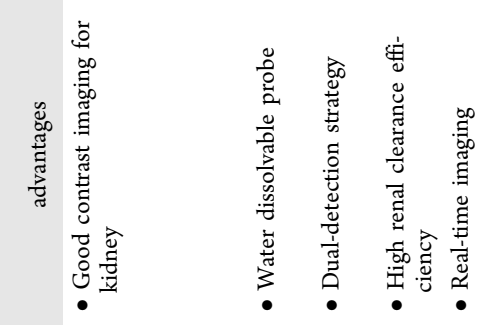

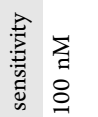

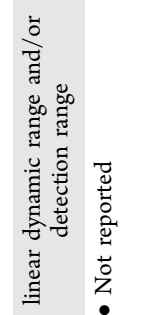

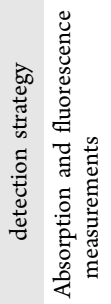

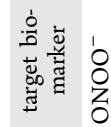

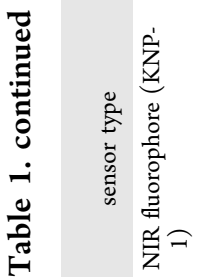

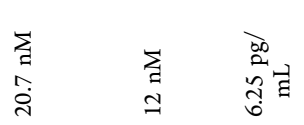
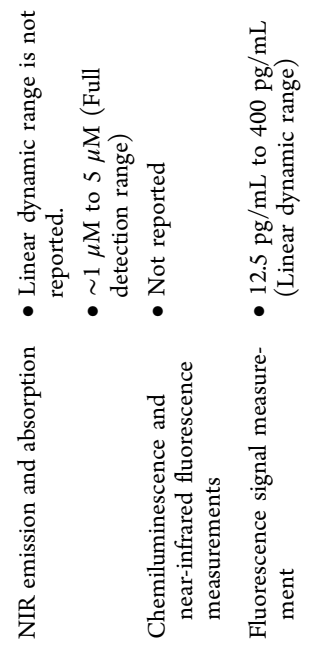

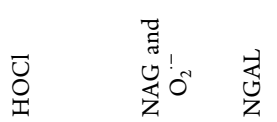

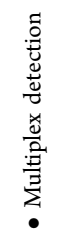

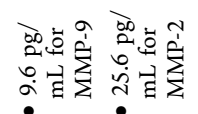

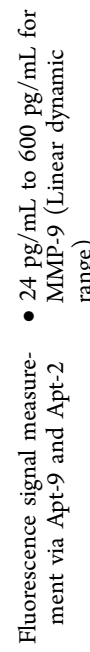

范

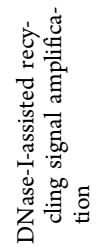

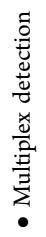

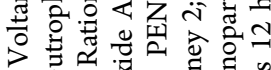

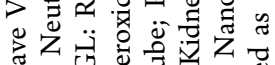

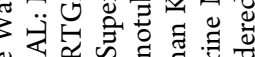

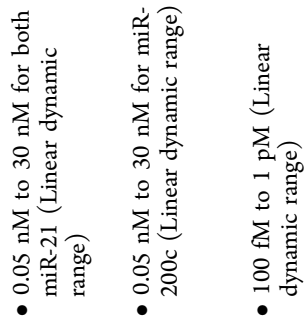

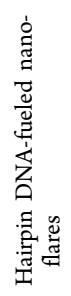

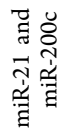

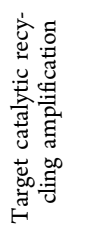

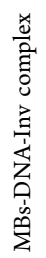

草

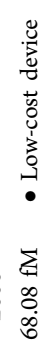

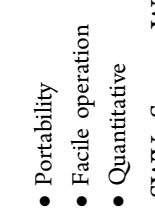

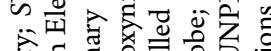

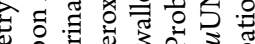

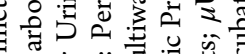

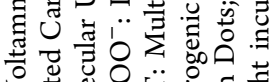

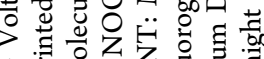

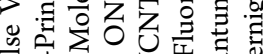

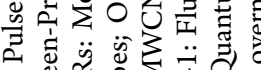

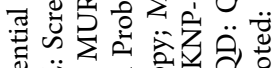

苛出可

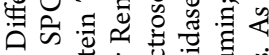

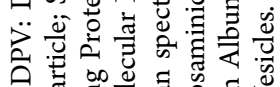

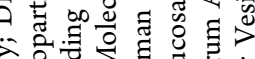

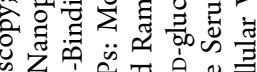

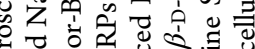

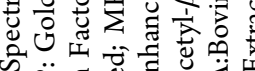

कित्र

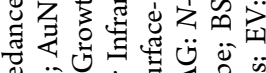

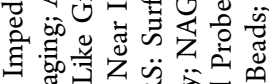

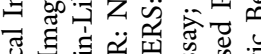

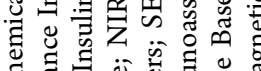

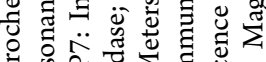

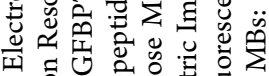

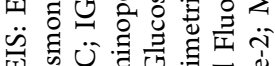

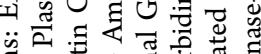

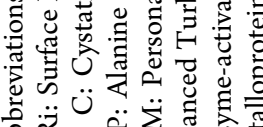



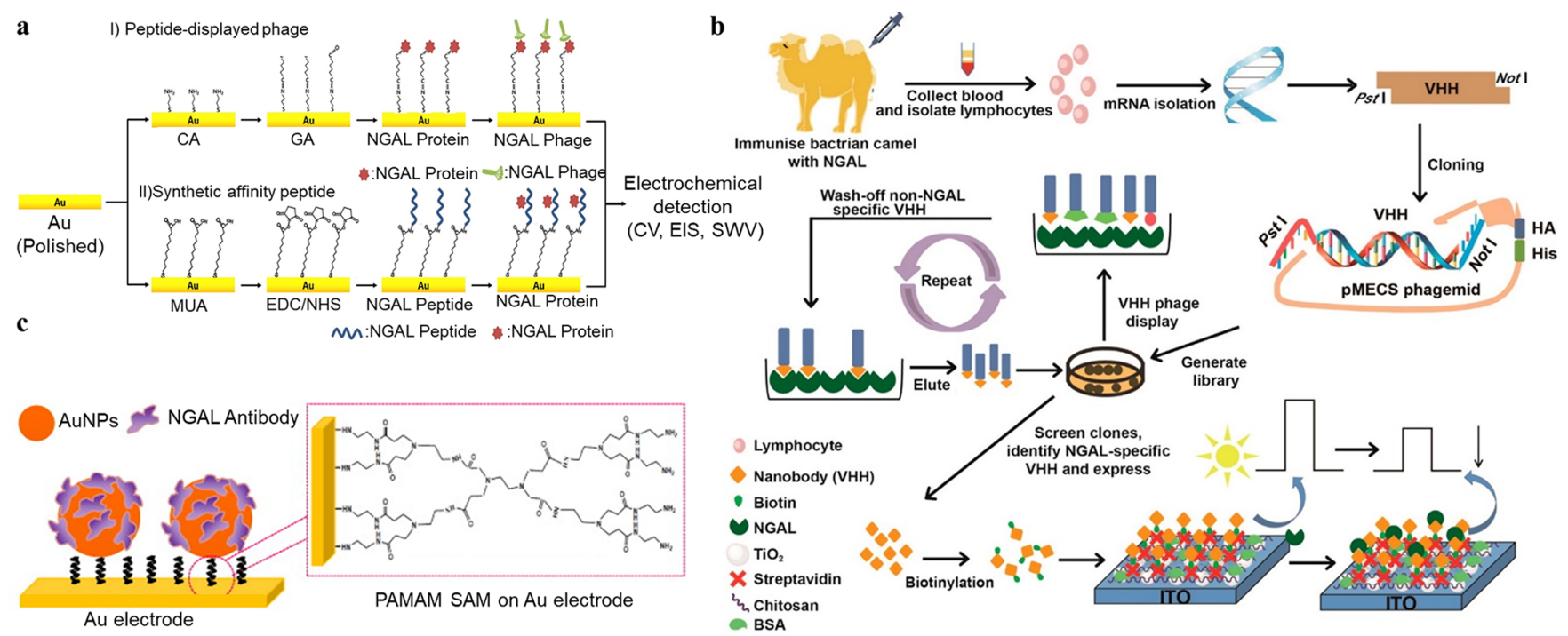

Figure 2. An electrochemical biosensor for NGAL detection. (a) A representative schematic shows an electrochemical biosensor with phage and peptide coating. Reproduced with permission from ref 56. Copyright (2019) Elsevier. (b) The workflow of a label-free PEC-based immunosensor utilizing anti-NGAL Nbs is exhibited. Reproduced with permission from ref 59. Copyright 2015, American Chemical Society. (c) A schematic of LA2/AuNPs/PAMAM-modified electrode is illustrated. Reproduced with permission from ref 60. Copyright (2012) Elsevier.

an electrical transducer is functionalized with bioreceptor molecules via a variety of chemical modifications such as amine, carboxyl, aldehyde, and thiol. ${ }^{48}$ After the functionalization, reactions are monitored through the generated current (amperometry) potential (potentiometry), or conductivity (conductometry). On the other hand, electrochemical impedance spectroscopy (EIS) also becomes prevalent by monitoring both resistance and reactance to monitor biochemical reactions for the detection. ${ }^{47}$

The analytical performance of biosensors is mainly evaluated through sensitivity, the limit of detection (LOD), linear dynamic ranges, reproducibility, precision of the response, selectivity, response time, operational and storage stability, ease-of-use, and portability. ${ }^{47}$ The improvement of these parameters is primarily performed by integration with functional nanomaterials, including carbon-based nanomaterials (e.g., CNTs, ${ }^{49}$ graphene $^{50}$ ) and metallic nanoparticles (e.g., gold nanoparticles, ${ }^{51}$ silver nanoparticles $\left.{ }^{52}\right) .{ }^{48}$ Electrochemical biosensors are applied in various fields, including clinics for disease diagnostics, ${ }^{53}$ food analysis, ${ }^{54}$ and environmental monitoring ${ }^{55}$ for pathogen detection. In developing a POC device, electrochemical-based biosensor systems are widely applied because of the features of cost-efficiency, rapid response, and low maintenance requirement and cost. ${ }^{56}$ This section presents electrochemical platforms developed for the AKI diagnosis by detecting different $\mathrm{AKI}$ biomarkers, including creatinine, NGAL, and Cys C. Different electrochemical biosensing approaches have been utilized to determine AKI biomarkers, but each of them has their own advantages and drawbacks with brief details that are listed in Table 1.

Creatinine (Cre) is the traditional biomarker for AKI determination. As an example, an electrochemical biosensor was developed through screen-printed gold electrodes (AuSPE) and molecularly imprinted polymer (MIP). ${ }^{57}$ To create an MIP structure for Cre molecules, the carboxylic polyvinyl chloride (PVC-COOH) layer was first employed on the AuSPE surface. Following that, acrylamide and $N, N^{\prime}$ methylene bisacrylamide were applied to the polymerization reaction. As a final step for the MIPs, Cre templates were removed. The analytical performance of the sensor was evaluated through three instrumental techniques: voltammetry (cyclic voltammetry (CV) and differential pulse voltammetry (DPV)), EIS, and UV-Vis spectrophotometry. Specificity analyses were investigated by applying urea and glucose, and they could not be captured by the MIPs. The LOD values were determined as $0.016 \mathrm{ng} / \mathrm{mL}$ for EIS and $0.081 \mathrm{ng} / \mathrm{mL}$ For DPV; and the linear detection was observed in a range from $0.1 \mathrm{ng} / \mathrm{mL}$ to 1 $\mu \mathrm{g} / \mathrm{mL}$ for EIS and DPV measurement. Performance evaluation was conducted with volunteers with different urinary Cre levels, and the results indicated that the developed sensor platform was promising for the detection of Cre. ${ }^{57} \mathrm{On}$ the other hand, serum Cre level was also used to investigate kidney function relying on enzymatic reaction. ${ }^{58}$ The principle of this biosensor was the conversion of Cre from 1methylhydantoin through a monoenzymatic pathway. Screenprinted carbon electrodes were modified with cobalt chloride as the chemical receptor to determine 1-methylhydantoin. 1methylhydantoin and cobalt formed a complex, which was then validated through optical absorption spectroscopy. The detection was within a range from 0.2 to $4 \mathrm{mg} / \mathrm{dL}$ in 300 $\mu \mathrm{L}$ of sample volume without any preprocessing for $3 \mathrm{~min}$. Consequently, this platform offers a new way of Cre measurements and demonstrates novelty, cost efficiency, and robustness when even interfering proteins (e.g., serum albumin) are present. Considering the aforementioned examples, the MIP-based biosensor is more efficient in terms of linear dynamic range.

Besides the detection of Cre, the other biomarkers were also investigated for determining AKI. For instance, another electrochemical biosensor was introduced to detect novel NGAL levels by using novel affinity peptides as the bioreceptor. ${ }^{56}$ As a note, NGAL is a renal injury biomarker for renal tubular damage, and it responds to tubular damage rapidly. ${ }^{24}$ In this study, synthetic peptides were designed, produced, and immobilized on the gold surface through covalent interactions in different concentrations in order to develop peptide-incorporated electrochemical sensors (Figure 2a). ${ }^{56}$ Novel affinity peptides that have the ability to bind 
NGAL were identified by the polyvalent phage display technique. Samples were examined to designate the highest specificity to NGAL by using EIS and square wave voltammetry (SWV), and the performance of the electrochemical phage sensor containing NGAL-specific peptides was analyzed by ELISA, CVs, SWV, and EIS. The validation of this sensor was performed through ELISA and EIS, and the researchers also tested crude plasma collected from the normal group, pre-diabetic patients, and diabetic patients. The ELISA results indicated that NGAL concentrations were elevated significantly in pre-diabetic and diabetic patients. As a result, the NGAL levels were determined as $21.09,42.34$, and 33.37 $\mathrm{ng} / \mathrm{mL}$ in normal, pre-diabetic, and diabetic groups, respectively. The peptide-incorporated sensor was able to detect NGAL accurately, and the LOD was determined as 1.74 $\mathrm{ng} / \mathrm{mL}$ and $3.93 \mathrm{ng} / \mathrm{mL}$ on EIS and SWV experiments, respectively. ${ }^{56}$

Continuing with the detection of NGAL, another electrochemical sensor was developed, but instead of using peptides, they integrated nanobodies (Nbs) onto a cobalt $\mathrm{TiO}_{2}$ electrode to create label-free photoelectrochemical (PEC) immunosensor. The electrode was prepared on indium-tinoxide (ITO) slices by using $\mathrm{TiO}_{2}$ nanoparticles and streptavidin-coated cobalt 2,9,16,23-tetraaminophthalocyanine, respectively (Figure $2 \mathrm{~b}$ ). ${ }^{59}$ The principle behind designing this sensor was Bactrian camel immunization with NGAL and phage-displayed library construction in order to create $\mathrm{Nbs}$ that have the highest affinity to NGAL. ${ }^{59}$ The concentrations of the NGAL and signal decrement have a vital relationship; therefore, sensitive, label-free biosensors were developed to monitor the immunoreaction extent of $\mathrm{BiNb}$ and NGAL based on the final photocurrent variations. Here, the NGAL concentration was determined based on the photocurrent decrease. The NGAL concentration and relative change of photocurrent provided a linear relationship within the range from $1 \mathrm{pg} / \mathrm{mL}$ to $500 \mathrm{ng} / \mathrm{mL}$, and the LOD value was around $0.6 \mathrm{pg} / \mathrm{mL}$. This platform was also compared with ELISA and localized surface plasmon resonance (LSPR)-based immunosensor and showed lower sensitivity. Sensitivity improvement could be performed in the immobilization step by achieving uniform antibody orientation and enhancing the antigenbinding sites for analytes. A specificity analysis was carried out by applying human chorionic gonadotropin (HCG), carcinoembryonic antigen (CEA), prostate-specific antigen (PSA), and $\alpha$-fetoprotein (AFP). The selectivity level of this sensor system was acceptable, and nonspecific adsorption was negligible. ${ }^{59}$ Instead of antibodies, Nbs were used, and they provided better thermostability. Furthermore, their application in immunoassay development will ensure longer shelf-life, presenting practical and feasible tests under harsh conditions. The effect of this sensor is promising for the AKI diagnosis with the fashions of simple operation, rapid response, and the potential for clinical application. ${ }^{59}$

An electrochemical-based sensing was developed to quantify NGAL levels from urine and blood, and this platform relied on antibody-immobilized gold nanoparticles (AuNPs) and a polyamidoamine (PAMAM) dendrimer layer-modified $\mathrm{Au}$ electrode. ${ }^{60}$ Electron-transfer relay between NGAL and electron surface was achieved through the AuNPs. One of the main benefits of combining AuNPs with a sensing platform was to enhance the capture performance by increasing the active surface area for the analytes. The functionalization of the PAMAM monolayer-modified Au electrode was carried out by immersing into the Au colloidal solution, and this electrode was incubated with the LA2 solution (Figure 2c). The attachment of the AuNPs was enabled through PAMAM dendrimers by creating strong immobilization strategy. Sensor performance was investigated through CV, DPV, and amperometric techniques and urine and serum samples were tested. As a results, higher than $97 \%$ of recovery rate was obtained. The detection limit was $50-250 \mathrm{ng} / \mathrm{mL}$, along with down to $1 \mathrm{ng} / \mathrm{mL}$ of LOD value. ${ }^{60}$ As mentioned by Kannan et al., oxidation peak current was based on electrochemical detection of NGAL through antibody-NGAL binding. Another recent study introduced a label-free immunosensor relying on graphene/polyaniline nanocomposite to detect NGAL, and that the coating of nanocomposite led to an increase in the oxidation current. ${ }^{61}$ Minimizing the working electrode (WE) size to manufacture portable biosensors could reduce the sensitivity due to a smaller detection area. Hence, graphene (G)/conducting polymer nanocomposite-modified electrodes were developed to increase the surface area. ${ }^{61}$ Electropolymerization was employed to produce G/PANI nanocomposite-modified electrodes. EDC/NHS surface chemistry was carried out to immobilize anti-NGAL capture antibodies, and NGAL detection was conducted by using $\mathrm{CV}$ and amperometry tools. Accordingly, G/PANI nanocompositemodified electrodes provided a $\sim 58$-fold rise in anodic peak current. The values of LOD and limit of quantification (LOQ) were determined as $21.1 \mathrm{ng} / \mathrm{mL}$ and $70.4 \mathrm{ng} / \mathrm{mL}$ under optimum conditions. The sensing platform was tested with urine samples from AKI patients and compared with the ELISA. The built-up biosensor showed a simple, time-efficient (e.g., $30 \mathrm{~min} / \mathrm{sample}$ ), and cost-efficient procedure, along with the requirement of lower sample volume (e.g., $10 \mu \mathrm{L}$ ); therefore, their applications into clinics would be promising for AKI diagnosis. ${ }^{61}$

As an alternative, the voltammetric sandwich-type electrochemical immunosensor platform was employed for the detection and quantification of NGAL. ${ }^{62}$ The sensor platforms were composed of a single-use, bare, screen-printed electrode (SPE), which was combined with an electrochemical cell comprising a dual WE as a transducer. One WE surface did not require any treatments for the functionalization, and also, in the same electrochemical cell, a negative control was implemented. ${ }^{62}$ A capture antibody was used for the second WE surface functionalization, and casein was used to block nonfunctionalized sites. Following that, NGAL and biotinylated detection antibody were subsequently applied. Streptavidin-alkaline phosphatase conjugate solution was added to the electrochemical cell to create a biotin-streptavidin interaction. Then, a 3-indoxyl phosphate/silver mixture was added onto the electrochemical cell surface for the enzymatic labeling. ${ }^{62}$ Enzymatically deposited silver was stripped voltammetrically, creating an analytical signal. LOD and LOQ values were determined as $0.096 \mathrm{ng} / \mathrm{mL}$ and $0.321 \mathrm{ng} / \mathrm{mL}$, respectively. The specificity/selectivity of the presented sensor was controlled by applying Cys $\mathrm{C}$ and human serum albumin (HSA) in the absence of NGAL, and the responses were similar to the blank, pointing out the specific recognition/ detection of NGAL. Furthermore, the presence of potentially interfering proteins (Cys $\mathrm{C}$ and HSA) did not cause any significant alterations for NGAL detection. ${ }^{62}$ The other advantages of this sensor platform were adequate analytical performance, small sample requirement, and a time-efficient procedure in urine. It provided a promising alternative for 
a

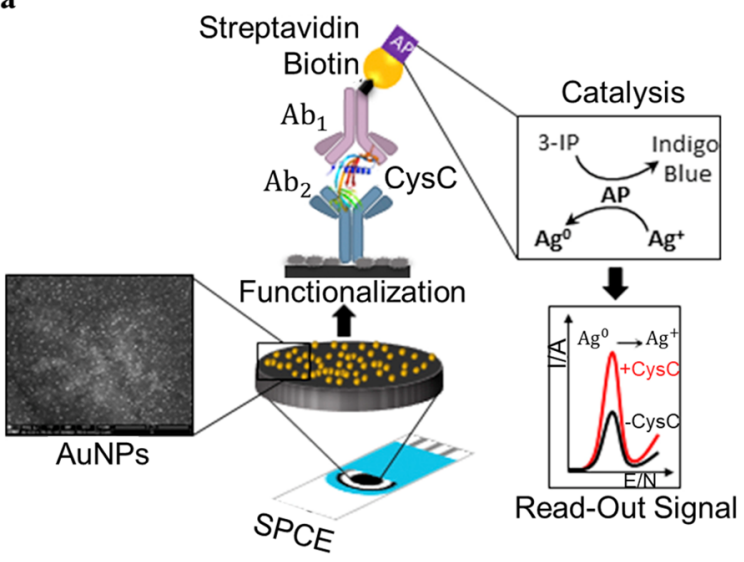

b

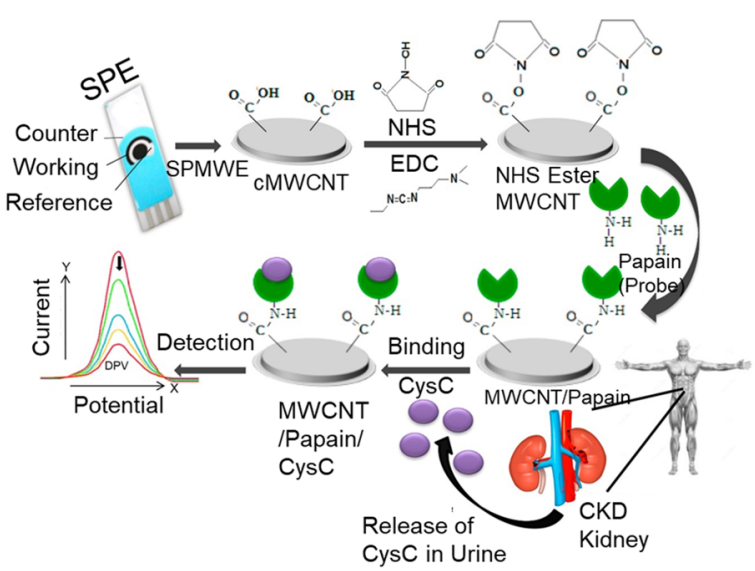

Figure 3. An electrochemical biosensor for Cys C detection. (a) The schematic of Cys C detection is presented. Reproduced with permission from ref 64. Copyright (2019) Elsevier. (b) The schematic illustration of Cys C detection from human urine sample and surface functionalization strategy is shown. Reproduced with permission from ref 65. Copyright (2017) Elsevier.

large-scale production of disposable and small-sized transducer. $^{62}$

In addition, $\mathrm{Cys} \mathrm{C}$ is another biomarker indicating renal functions, so kidney dysfunction can be determined by monitoring this protein. However, existing analytic strategies (e.g., ELISA, turbidimetric and nephelometric immunoassays) for serum Cys $\mathrm{C}$ detection are time-consuming, and they require highly qualified personnel. Therefore, several studies are carried out to develop biosensors and immunosensors for rapid detection of Cys $\mathrm{C}$. ${ }^{63}$ For instance, redox probe-free electrochemical immunosensor employing a catalytic nanocomposite made of graphene oxide (GO) functionalized with aminoferrocene $(\mathrm{Fc})$ was utilized. Three electrode systemscounter electrode (helical platinum wire), reference (Ag/ $\mathrm{AgCl}$ ), and working gold electrode (GE)-were placed to the site, where the electrochemical measurements were taken. Anti-Cys C antibodies were immobilized on the modified GE surface, and free adsorption-reactive sites were blocked through the glycine incubation. SWV was used for the electrochemical detection of Cys C. ${ }^{63}$ The role of the polyethylenimine (PEI) is essential, since it has a significant amount of amine groups that act as a cross-linker, and hence, these amine groups attached to the GO via amide bonds while leaving the free amine region for antibody attachment from carboxylic groups of the Fc region. The analytical performance of the platform was assessed by tracking the current differences before and after the application of Cys $\mathrm{C}$ solution. The results indicated a decrease in cathodic peaks as higher concentrations of Cys $\mathrm{C}$ were applied. ${ }^{63}$ The LOD value and response range of this system were $0.03 \mathrm{ng} / \mathrm{mL}$ and 0.1 to $1000 \mathrm{ng} / \mathrm{mL}$ of Cys $\mathrm{C}$, respectively. Moreover, the sensor platform showed good accuracy, specificity, and achieved good sensitivity for the detection of Cys $\mathrm{C}$. The major benefits of this sensor approach were facile use of an intrinsic redox probe and rapid testing with very few steps. Therefore, working in a reduction potential enabled potential interference avoidance. Amperometric detection had limitations; however, the proposed approach above would overcome these limitations, and it was considered a promising candidate for redox probe-free POC diagnostics tools in the future. ${ }^{63}$ Another electrochemical immunosensor was introduced to detect Cys $\mathrm{C}$ by modifying gold nanoparticles (Figure $3 \mathrm{a}$ ). The procedure was employed using screen-printed electrodes and the sandwich immuno- assay principle. The linear range was determined as 10 and 100 $\mathrm{ng} / \mathrm{mL}$, as well as LOD and sensitivity values were $6.0 \mathrm{ng} / \mathrm{mL}$ and $6.4 \pm 0.3 \mu \mathrm{A} \mathrm{ng} \mathrm{mL}{ }^{-1} \mathrm{~cm}^{-2}$, respectively. ${ }^{64}$ On the other hand, various nanomaterials like multiwalled carbon nanotubes (MWCNTs) have been integrated to electrochemical biosensors. For instance, an electrochemical biosensor was developed to quantify Cys $\mathrm{C}$ levels by combining an electrode surface with carboxyl functionalized MWCNTs, and it was immobilized with papain (cysteine protease) through EDC/ NHS chemistry (Figure 3b). The main function of CNTs in electrochemical biosensors is to enable efficient electron transfer between immobilized proteins and the electrode, thereby improving sensitivity, specificity, and stability parameters. CV and DPV were used to determine the interactions between papain and Cys $\mathrm{C}$ molecules. Consequently, the platform provided $0.58 \mathrm{ng} / \mathrm{L}$ of LOD, and sensitivity was measured as $1583.49 \mu \mathrm{A} \mathrm{cm} \mathrm{cm}^{-2} \mu \mathrm{g}^{-1}$, holding a pivotal potential as a portable device for AKI diagnosis. ${ }^{65}$ Recently, a study combined MIP and carbon-stemmed SPEs to determine Cys C levels. ${ }^{66}$ Creating molecularly imprinted poly(Py) matrix (MPPy) on the electrode enabled a rapid and simple procedure, as well as resulting in good reproducibility, accuracy, and linearity within the biomarker concentrations. During the polymerization, pyrrole ( $\mathrm{Py}$ ) was used to improve electrocatalytic properties of the electrode. Integrating MWCNTs formed more porous and larger surface area; therefore, 3D MPPy structures could be attained with enhancements in the sensitivity. The concentration of Cys $\mathrm{C}$ was determined via $\mathrm{CV}$ and DPV, and the LOD was quantified as $0.5 \mathrm{ng} / \mathrm{mL}$ at $\mathrm{pH} 6.0$ along with a linear dynamic range from 0.5 to $20.0 \mathrm{ng} / \mathrm{mL}$ when $20 \mathrm{~min}$ incubation was applied. ${ }^{66}$

\section{PLASMONIC-BASED SENSOR SYSTEMS}

Most of the state-of-the-art plasmonic biosensors measure binding affinity and monitor kinetic rates in real-time through the tracking of the refractive index (RI). ${ }^{67}$ Plasmonic resonance nanostructures have attracted notable interest in the scientific community to develop biosensors due to sensitive detection capability of biomolecules in biological fluids. The resonance condition is the main parameter determining sensitivity and specificity, while design, fabrication, and optimization of plasmonic substrates require substantial 
a

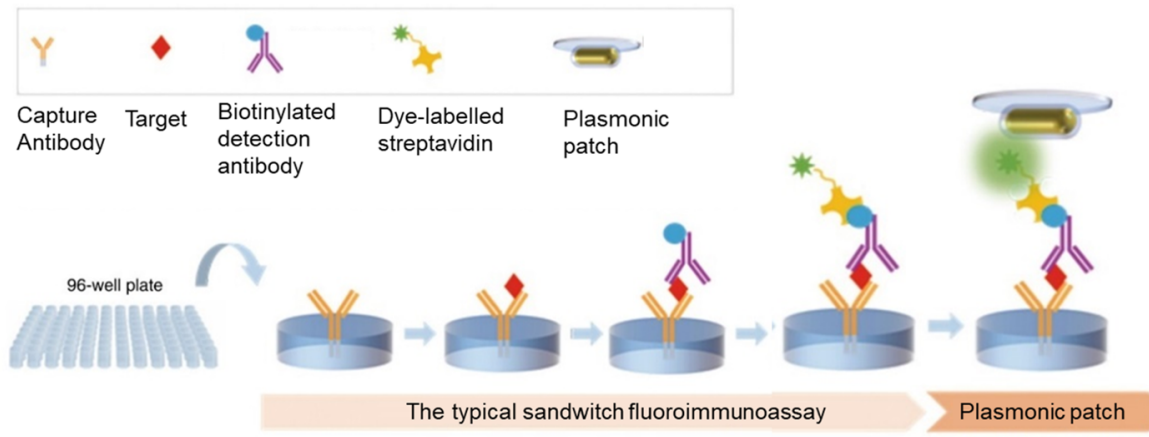

b

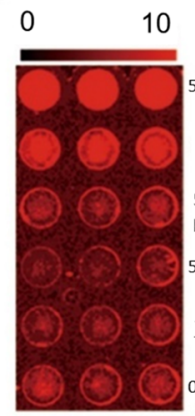

Unenhanced

d

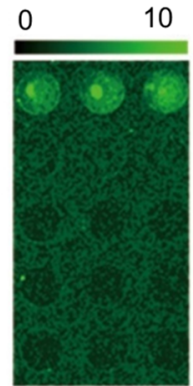

Unenhanced
50

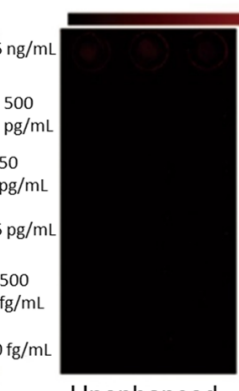

Unenhanced

100
500

c

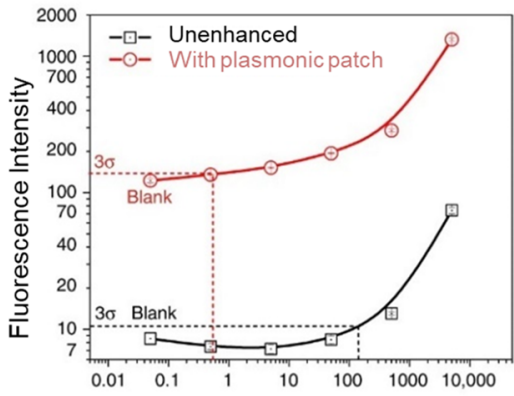

$\mathrm{KIM}-1$ concentration $(\mathrm{pg} / \mathrm{mL})$
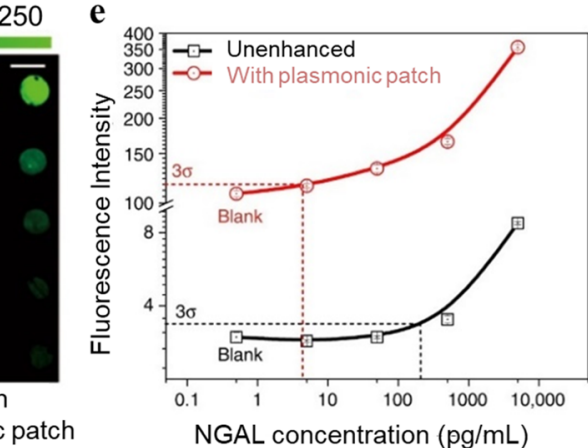

Figure 4. (a) The schematic represents the application plasmonic patch. Similar to a conventional ELISA, the platform utilizes a 96-well plate made of glass-bottom surfaces that are functionalized with aldehyde. Dye-labeled streptavidin is then added to the wells, and the plasmonic patch is transferred to the last step of the fluorescence enhancement. $(b-d)$ Fluorescent enhancement via plasmonic patch application is compared with the unenhanced assay. In this comparison, KIM-1 and NGAL are evaluated. (c-e) Fluorescence intensities are indicated with respect to the concentrations of KIM-1 and NGAL with (red line) and without (black line) the addition of the plasmonic patch. With this configuration, the LOD values of the KIM-1 and NGAL are improved $\sim 300$-fold and $\sim 100$-fold, respectively. Reproduced with permission from ref 82 . Copyright (2018) Nature.

attention. Although their development is commonly executed using metals, novel integrative materials such as graphene and its derivations ${ }^{68,69}$ have also drawn attention. ${ }^{70}$ Surface plasmon resonance (SPR), LSPR, and surface enhanced raman spectroscopy (SERS) have been widely applied as optical biosensor systems for disease detection. ${ }^{70-72}$ The strength of the SPR and LSPR in clinical diagnosis, environmental monitoring, and food safety as biosensing platforms comes from rapid and ultrasensitive detection of the diverse analytes (e.g., viruses, ${ }^{38}$ bacteria, $^{73}$ and proteins ${ }^{74,75}$ ). ${ }^{67}$ In addition, SERS promises label-free, sensitive, and selective detection by utilizing amplified localized electric fields on the surface of a plasmonic material through exciting monochromatic light. ${ }^{76}$ Currently, SPRs have been employed to detect and monitor biomolecular markers, including cancer markers, antibodies, drugs, and hormones for diverse medical conditions like Alzheimer's, hepatitis, diabetes, leukemia, and cancers such as prostate and breast cancers. ${ }^{35,38,74,77-81}$ SPR-based bio- sensors have applications in the development of POC devices for AKI diagnosis. This section demonstrates plasmonic biosensors for AKI diagnosis by detecting different AKI biomarkers, including KIM-1, NGAL, serum albumin, IGFBP7, and Cys C.

Starting with recent examples in the field, the researchers introduced a plasmonic patch enabling fluorescence enhancement on different surfaces; therefore, existing assay protocols do not require any modifications except for the addition of the patch in contrast to existing fluoroimmunoassay methods. ${ }^{82}$ The transfer of the patch did not require any training due to a facile application. Fluorescence enhancement (100-fold) was achieved by the plasmonic patch owing to the enhanced electromagnetic field, also resulting in $~ 300$-fold improvement in the sensitivity. ${ }^{82}$ The plasmonic patch used in this study was a transparent elastomeric film containing metal nanostructures, hence enabling the enhancement in fluorescence measurements by conformal contact (coming closer to fluorescent 


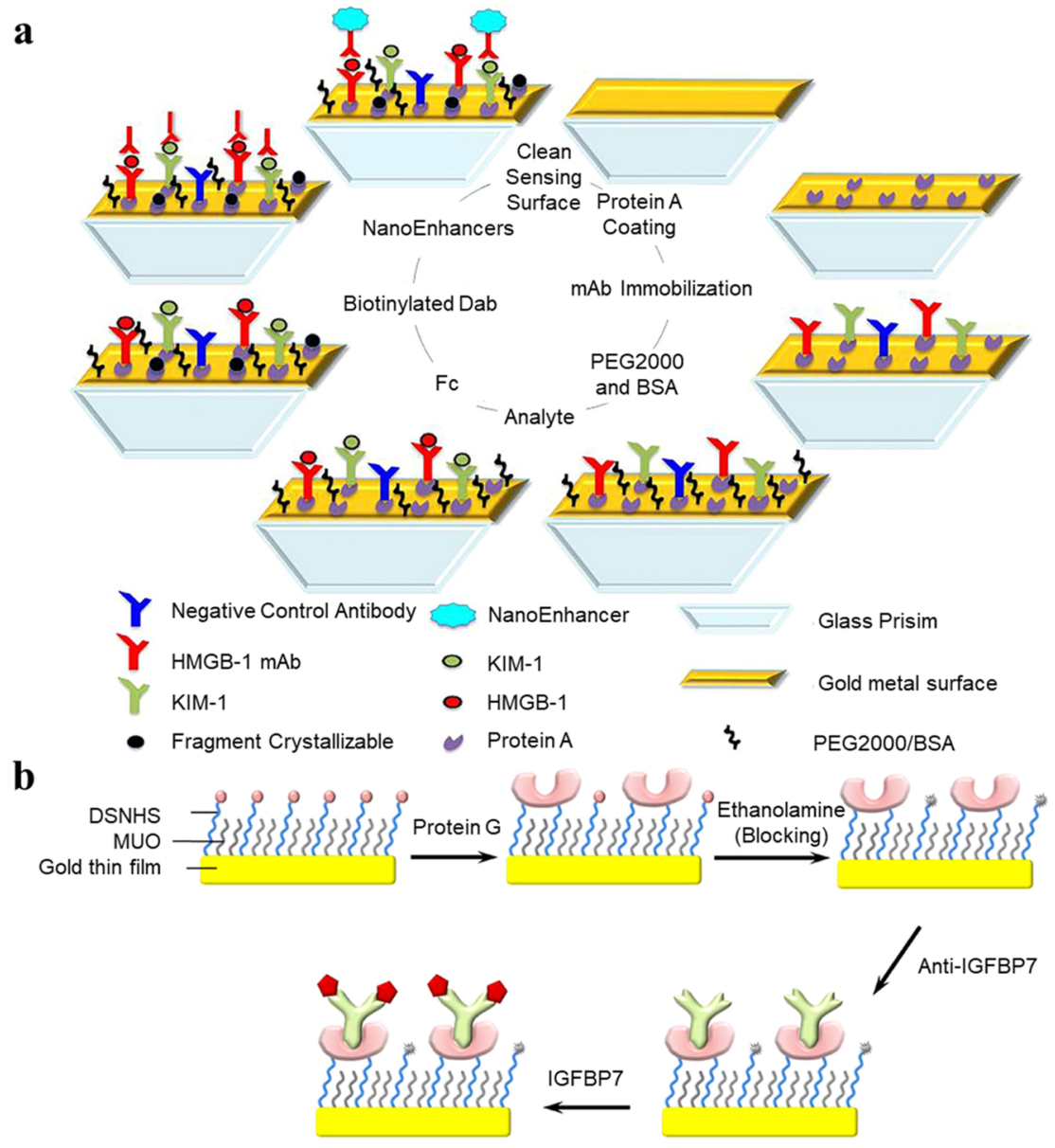

Figure 5. (a) An illustration of the KIM-1 and HMGB-1 detection via SPR-based biosensor is shown. Reproduced with permission from ref 83 . Copyright (2016) Nature. (b) IGFBP7 detection via SPR-based biosensor is illustrated. Reproduced with permission from ref 88 . Copyright (2014) Elsevier.

species in assay) with fluorescence (Figure 4a). The performance was tested with the detection of KIM-1 and NGAL (Figure $4 \mathrm{~b}-\mathrm{e}){ }^{82} \mathrm{KIM}-1$ is another novel biomarker for AKI detection, since the injury leads to elevation of KIM-1 levels in both urine and kidney within a short time, holding a potential for early diagnosis of kidney injury. ${ }^{28}$ In this study, the patch was fabricated from a polydimethylsiloxane (PDMS) elastomer. A negatively charged surface was attained after poly(styrenesulfonate) PSS treatment; therefore, positively charged plasmonic nanoparticles were absorbed via electrostatic interactions. ${ }^{82}$ Besides silicon, diverse surfaces such as glass, nitrocellulose, and polystyrene were investigated, and the researchers claimed that this strategy would be independent from the surface and showed multiple applications in fluorescence-based detection, quantitative sensing, and imaging techniques. ${ }^{82}$ Another parameter influencing the performance was the distance between plasmonic nanostructures and fluorophores, which was also optimized by creating a spacer layer with varying thickness by the formation of polysiloxane copolymer film. While increasing the spacer thickness, gradual red-shifts of the longitudinal LSPR wavelength of gold nanorods (AuNRs) were observed, since the RI of the medium surrounding the nanostructures changed. ${ }^{82}$ This add-on plasmonic patch enabled an improvement in bioanalytic parameters, including sensitivity, LOD, and dynamic range when the fluoroimmunoassays (e.g., 96-microplate format or antibody microarray) were carried out. The most crucial benefit of this platform was reliable performance when different assay formats, target biomarkers, and fluorophores were tested. Moreover, the established assay procedure did not require any additional training and any modifications on the read-out device. ${ }^{82}$ Recently, a label-free SPRi-based nanoimmunosensor was introduced to detect KIM-1 in real-time. ${ }^{83}$ This system offered multiplex and quantitative detection in buffer solutions. The surface of biosensor was modified with protein $\mathrm{A}$, and then, anti-KIM-1 antibodies $(50 \mu \mathrm{g} / \mathrm{mL})$ and anti-HMGB-1 antibodies $(50 \mu \mathrm{g} / \mathrm{mL})$ were decorated on this surface to achieve proper orientation. Nonspecific binding was minimized through sequential blocking, which was composed of polyethylene glycol (PEG2000) and bovine serum albumin (BSA). Followingly, a sandwich assay was conducted with the biomarker, an fc fragment (blocking unoccupied protein A), biotinylated secondary antibody, and, finally, streptavidinconjugated NanoEnhancers (NIR quantum dots), which amplified the signal (Figure 5a). The LOD value was determined as $5 \mathrm{pg} / \mathrm{mL}$ for KIM-1 and HMGB-1 in buffer. ${ }^{83}$

The recognition elements employed in plasmonic biosensors are mostly antibodies; however, their relatively unstable structure, expensive production, and limited robustness lead to seeking new biorecognition elements to eliminate or minimize these challenges. ${ }^{84}$ Molecular imprinting technique and gold nanocages (AuNCs) to create built-in artificial antibodies for detection of NGAL from body fluids were combined to enhance the sensitivity and specificity of the 
diagnostic process of AKI. ${ }^{85}$ AuNCs were preferred owing to their higher RI sensitivity, enabling a lower LOD value in comparison to the rod or spherical structures. AuNCs with porous sidewalls were synthesized by the galvanic replacement of $\mathrm{Ag}$ nanocubes, which were obtained through a sulfidemediated polyol synthesis method, using chloroauric acid $\left(\mathrm{HAuCl}_{4}\right)$. After obtaining AuNCs, a molecular imprinting technique was performed to specify nanocages for NGAL. ${ }^{85} \mathrm{~A}$ molecular imprinting procedure was composed of reversible immobilization of biomolecules on AuNCs via $p$-aminothiophenol (p-ATP) and glutaraldehyde (GA) cross-linkers. ${ }^{85}$ In the end, a highly sensitive plasmonic NGAL biosensor was obtained regarding the high RI sensitivity of AuNCs with a specialized surface. The reversible bonds between templates and AuNCs were broken, and hence, the selective binding of NGAL to the built-in artificial antibodies was achieved in the presence of physiological fluids containing interfering proteins. The LOD value was determined as $25 \mathrm{ng} / \mathrm{mL}$ with high selectivity when numerous interfering urinary proteins were present. Furthermore, the sensing platform exhibited good stability against a wide $\mathrm{pH}$ range (from 4.5 to 8.5 ). The built-in artificial antibodies were shown to be promising for POC applications in clinics for the diagnosis of AKI. ${ }^{85}$

Another sensing platform was the SPR biosensor to detect HSA. $^{86}$ The methodology can be summarized as the fabrication of the chip, self-assembled monolayer preparation, Cibacron Blue F3GA (CB) immobilization, characterization of PEG-CB monolayer, and SPR spectroscopic measurements. The interactions between PEG-CB and HSA were monitored by a home-built SPR biosensor designed according to the Kretschmann configuration using attenuated total reflection (ATR) and flow analysis system. The platforms mentioned above utilized nanoparticles for the signals. Their measurement was carried out via the stabilization of carrier buffer, loading samples, and washing with the same carrier buffer, respectively. Specificity analysis of the sensor platform for PEG-CB monolayer to HSA was determined by using lysozyme (nonspecific adsorption control). The results displayed that the PEG-CB surface was specific to HSA since the signal difference could distinguish HSA from lysozyme. ${ }^{86}$ In order to estimate the capability clinical diagnosis, the range of the HSA concentration was designed as 0.01 and $0.1 \mathrm{mg} / \mathrm{mL}$, since the normal physiology range is lower than $0.02 \mathrm{mg} / \mathrm{mL}$ (physiological range) and the microalbuminuria range is defined between 0.02 and $0.1 \mathrm{mg} / \mathrm{mL}$. The linear increments proportional to the concentrations were observed $(R=0.998$, $P<0.0001)$. Detection limits were designated as $4 \mu \mathrm{g} / \mathrm{mL}$. In sum, this sensing platform could detect the changes in HSA concentration in normal physiology and microalbuminuria range. $^{86}$

In addition, insulin-like growth factor-binding protein 7 (IGFBP7) is a member of the IGFBP superfamily and highly associated with kidney injury. It is considered a secondgeneration biomarker for AKI detection with a tissue inhibitor of metalloproteinases-2 (TIMP-2). ${ }^{17}$ The primary role of IGFBP7 is to regulate the bioavailability via direct low-affinity binding of IGFs, since they have a pleiotropic property in diseases. ${ }^{87}$ As an example, in order to detect IGFBP7, the SPRbased platform was developed. ${ }^{88}$ The gold surface of the sensor was functionalized with anti-IGFBP7 antibodies through mercaptoundecanol (MUO), and DSNHS $\left(\mathrm{S}_{2}\left(\mathrm{CH}_{2}\right)_{11} \mathrm{COO}-\right.$ $\left(\mathrm{CH}_{2}\right)_{2} \mathrm{COO}-(\mathrm{N}$-hydroxysuccinimide $\left.)\right)$ and protein $\mathrm{G}$ immobilization. Before anti-IGFBP7 antibody immobilization, non- specific interactions were minimized through ethanolamine blocking (Figure 5b). The LOD value and linear dynamic range were determined as $10 \mathrm{ng} / \mathrm{mL}$ and $10-300 \mathrm{ng} / \mathrm{mL}$, respectively. The specificity of the sensor was controlled by applying interleukin (IL) derivatives (e.g., IL-4, IL-23, IL-29). Comparing the results with the gold standard method such as ELISA would be more beneficial to understand its utility in clinical diagnosis.

Overall, electrochemical and plasmonic biosensors have been widely applied to detect target analytes. One recent study aimed to combine these two approaches for developing a spectroelectrochemical nanosensor. The dual modalities were a combination of surface-enhanced Raman spectroscopy and differential pulse voltammetry (SERS/DPV) to detect Cys C levels from blood plasma. ${ }^{89}$ The system was an extractor chip (made of gold-coated silicon chip) functionalized with thiolated antibodies to isolate protein from blood plasma. SERS and DPV measurements provided $1 \mathrm{pM}$ and $62.5 \mathrm{nM}$ detection, respectively. Consequently, this sensor detecting Cyc C could be employed for kidney disease monitoring. ${ }^{89}$

\section{INTEGRATABLE SYSTEMS}

The advancement of nanotechnology has enabled various insights into the biosensing realm, and in particular, different kinds of nanomaterials, such as carbon-based nanomaterials, nanoparticles, 2D materials, and quantum dots, have been integrated with them. ${ }^{90}$ In addition to the material development, various fabrication techniques were developed; therefore, small fluid manipulation devices such as microfluidics were integrated with them. ${ }^{91-97}$ Both nanomaterials and microfluidic devices well-harmonized with biosensors to enhance their analytical performance, as well as to improve the cost and assay time-related issues. For instance, graphene, one of the most employed nanomaterials in biosensing, increases the surface area of electrodes, allowing higher recognition immobilization and eventually providing larger recognition sites for the binding of target molecules. ${ }^{98}$ Microfluidics requires only a small amount of reagent consumption and helps miniaturizing the system to be as more compact as possible. ${ }^{36,74,99-102}$ Consequently, this section presents brief informative and integrative features of nanomaterials (mostly nanoparticles) and microfluidic platforms to biosensors for AKI diagnosis.

At first glance, nanoparticles (NPs) are among the most diverse classes of nanomaterials that exhibit various optical, electronic, and magnetic properties in comparison to bulk materials. Considering these properties, they have been widely utilized in biosensor development for clinical applications. The main benefit of the NPs is altering the physicochemical properties by changing the shape and size. Large surface-areato-volume ratios of NPs allow for higher biomolecule attachment; therefore, sensitivity enhancement and improvements in LOD value can be achieved. ${ }^{103}$ Gold NPs, ${ }^{104}$ silver NPs, ${ }^{52}$ magnetic NPs, ${ }^{105}$ quantum dots, ${ }^{106}$ polymeric NPs, carbon nanotubes, and nanodiamonds are largely employed NPs in biosensing systems, such as lateral flow immunoassays, $^{107}$ colorimetric $^{108}$ and fluorescence ${ }^{109}$ detection, and electrochemical, ${ }^{110}$ SERS, ${ }^{111}$ plasmonic, ${ }^{112}$ mechanical, ${ }^{113}$ and micronuclear magnetic resonance ${ }^{114}$-based platforms. For instance, MNP-based diagnostic tools have gained significant interest in developing biosensing platforms for various human diseases. In this regard, MNPs are involved as labels for the target molecule (e.g., cells or extracted molecular compo- 


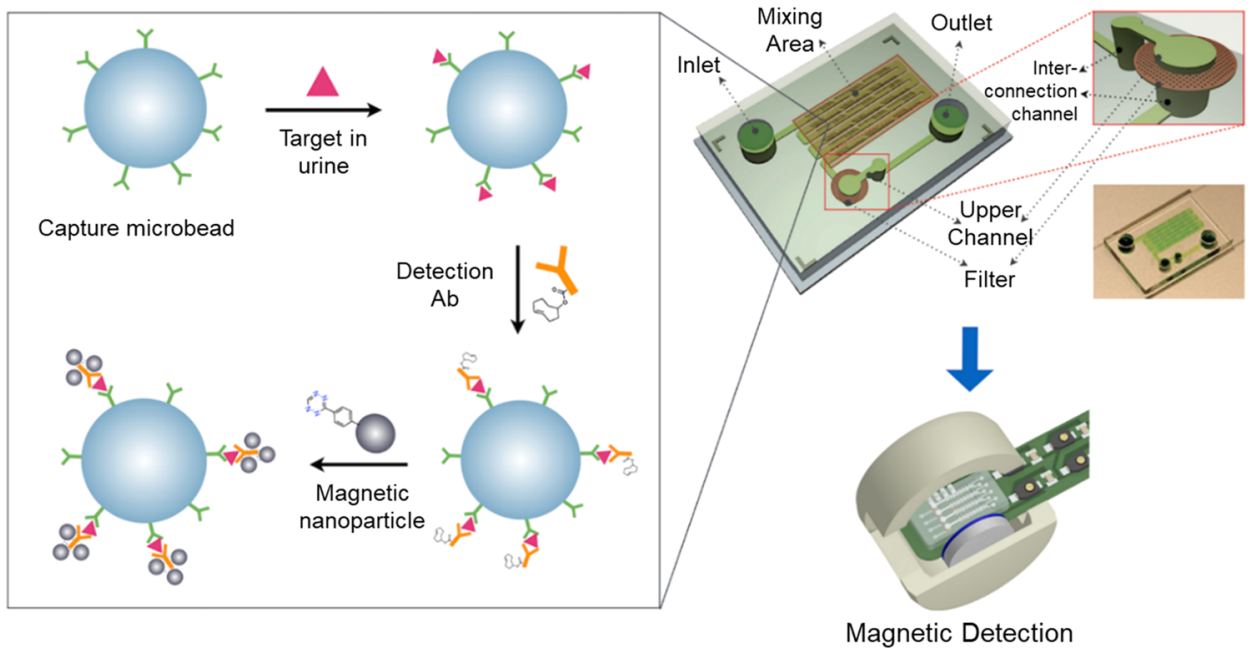

Figure 6. The $\mu \mathrm{UNPD}$ and its workflow are represented. Reproduced with permission from ref 105. Copyright (2015) Public Library of Science.

a

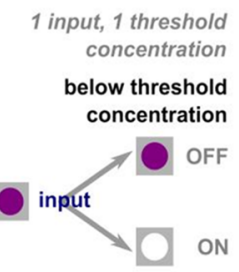

above threshold concentration

b

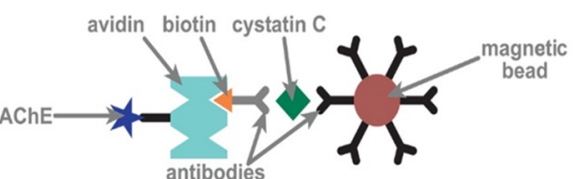

c

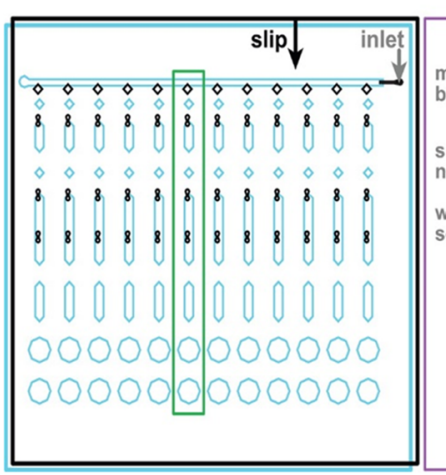

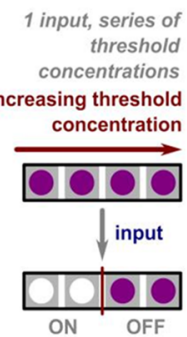

d

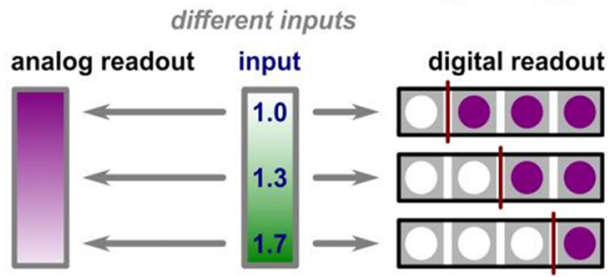

$\mathbf{e}$

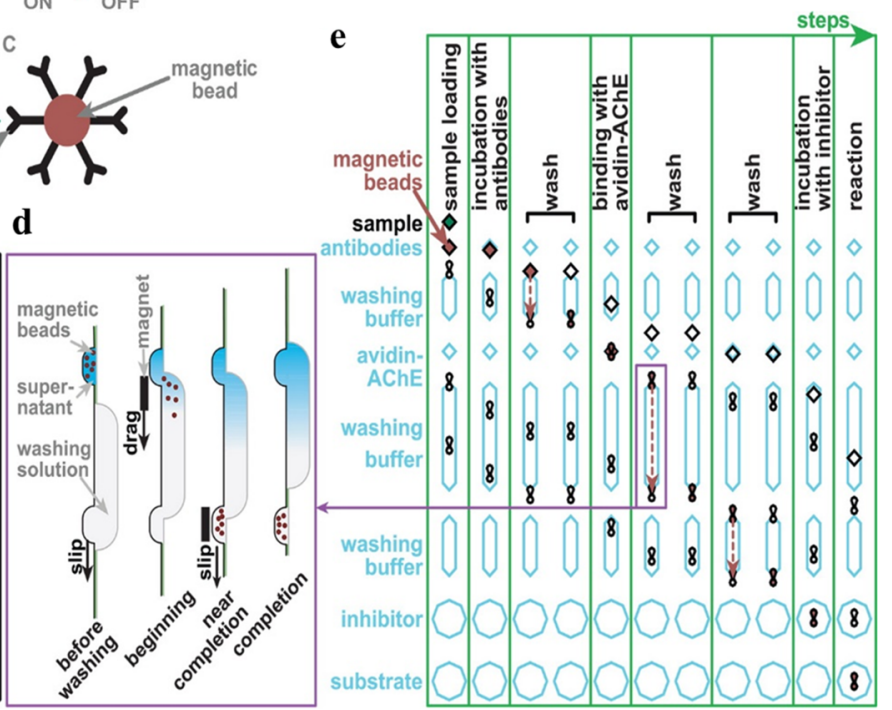

Figure 7. (a) The threshold chemistry is represented. In high-input concentration (above the thresholds), the platform provides ON response. The ON/OFF transition for simple read-out is also represented. Reproduced from ref 120. Copyright (2013) American Chemical Society. (b) Mbintegrated platform is designed for the detection of Cys C. (c) The SlipChip design composes of top plate (black lines) and bottom plate (blue lines) with 12 columns that have different threshold concentrations. The inlet indicates the sites for sample loading. (d) The washing steps, in which magnetic beads are moved through the channel. (e) The assay is illustrated according to the steps. Reproduced from ref 120 . Copyright (2013) American Chemical Society.

nents). For instance, a miniaturized nuclear magnetic resonance device $(\mu \mathrm{NMR})$, which was procured with high sensitivity by eliminating low background signals, was presented to detect the labeled target molecules. ${ }^{105} \mathrm{~A}$ semiautomated, microurine, nanoparticle detection ( $\mu \mathrm{UNPD})$ technique was developed to sense minute amounts of KIM-1 and CysC from urine samples through the labeling with nanoparticles. The basic principle of this platform was the utilization of automated on-chip assay and detection by hand- held device for the read-out. ${ }^{105}$ The $\mu \mathrm{UNPD}$ was produced through (i) the microfluidic device fabrication on a glass slide by stacking three layers of PDMS and (ii) the preparation of assay reagents that included the preparation of material made from carboxylated polystyrene and activation of the capture beads via EDC/sulfo-NHS solutions. The activated capture beads were then mixed with polyclonal antibodies targeting KIM- 1 and Cys C. The mechanism of the $\mu$ UNPD could be described as blending capture beads, which were coated with 
antibodies (both for KIM-1 and Cys C) and incubated with the target solution. The unbound targets were then washed out with PBS. Later on, the capture beads and detection antibodies were reunited, and another washing step was carried out to remove unbound antibodies. Then, MNPs were added to acquire labeling, and the last washing step finalized the process. To achieve the automation, all the materials (e.g., capture beads, target solution, detection antibodies, and magnetic nanoparticles) were placed on the inlet of the microchip in an order, and negative pressure was applied in the channels for the mixing. Microchip contains a filter membrane, which acted as a barrier for retaining beads during the flow of the solution. A $\mu$ NMR device was used for the measurements. ${ }^{105}$ Figure 6 also summarizes the overall mechanism of $\mu \mathrm{UNPD}$ assay. In practice, KIM-1 and Cys $\mathrm{C}$ are urine biomarkers that are conventionally examined by complex instruments (e.g., Luminex). Considering bulky systems, multiple labeling agents, and sophisticated instruments, the $\mu \mathrm{UNPD}$ technique provided a facile alternative and reduced turnaround time and the need for expensive reagents and specialized personnel for the proper measurements. However, this technique holds some drawbacks, including the paucity of a quantitative readout system. ${ }^{105}$

Besides, quantum dots (QDs)-based detection systems can be used to advance the current detection platforms for AKI biomarkers and to improve the practical impediments (e.g., such as high-cost and lengthy assay time) at the POC settings. ${ }^{106}$ Nowadays, fluorescence at NIR range is preferred in comparison to fluorescence in the visible light range due to possible deep penetration into biometrics, less photodamage, weak scattering of emission light, and lower fluorescence background. The attention and examples (e.g., $\mathrm{Ag}_{2} \mathrm{~S}, \mathrm{CdTe} /$ $\mathrm{CdS}, \mathrm{AgInSe}_{2} / \mathrm{ZnS}$, or $\mathrm{CdTeSe} / \mathrm{ZnS}$ ) of QDs and NIR combination have been increased over the years in biomarker detection for diseases or biomedical imaging. PbS@BSA QDsbased assay and imaging systems at NIR range were proposed in detecting Cys $\mathrm{C}$. The working principle of this system is based on the specific affinity between $\mathrm{Cys} C$ and papain, which is a cysteine protease enzyme that hydrolyzes the BSA, and it is inhibited by Cys C. The digestion of the BSA by papain was used to synthesize PbS@BSA QDs, and the fluorescence intensity decreased step-by-step considering to the digestion. The determination of Cys $\mathrm{C}$ was the proportional to papain inhibition, and accordingly, the LOD value was observed down to $0.02 \mathrm{mg} / \mathrm{mL}$. $^{106}$

Elaborating on the latter integrative perspectives, microfluidics also offers notable benefits over conventional techniques in terms of rapid turnaround, high throughput, and cost aspects to investigate and manipulate the behavior of fluids at a small scale. ${ }^{115-118}$ Recent advances in microfluidics and nanotechnology have denoted simple, rapid, accurate, portable, multiplex, and inexpensive solutions for disease diagnosis. ${ }^{80,119}$ In biological analysis, microfluidics has huge applications due to fluid manipulation such as sample enrichment, sorting, and isolation at a microscale. The combination of microfluidics with different kinds of sensor systems enables the development of portable, stable, and robust methods for label-free detection. ${ }^{36}$ For the AKI detection, an NP-integrated microfluidic platform called SlipChip was developed to enable multiplexing and miniaturization for the implementation of the analog-to-digital chemical signal conversion, which was an approach to quantify Cys $\mathrm{C}$ through a visual read-out mechanism (Figure $7 \mathrm{a}-\mathrm{e}$ ). ${ }^{120}$
Chemical signals digitized the analog data with respect to concentration changes in the on/off form. The analog-todigital conversion was achieved by the threshold chemistry based on the input concentration. Briefly, the threshold chemistry relies on color change when the input concentration is higher than that of the predetermined concentration. The principle of threshold chemistry utilizes enzymatic inhibition (competitive inhibition). Briefly, the enzyme is initially incubated with a competitive inhibitor, and then, it reacts with the substrate. Once the input (enzyme) concentration is altered from lower inhibitor concentration to slightly higher inhibitor concentration, output concentration, which is available active enzyme, increases considerably. The tight binding mechanism between inhibitor and enzyme leads to a more considerable increase in the output concentration. Overall, threshold chemistry would be applied to analog-todigital conversion with four requirements; (i) ability to detect increases in concentration with desired sensitivity, (ii) tunability for different thresholds, (iii) simple visual readability (e.g., naked-eye detection), and (iv) possible usage for the other assays. ${ }^{120}$ Regarding the assay workflow, when the threshold is higher than the input concentration, an OFF response is obtained from the reaction in one bit and vice versa. The ON response is achieved in the form of a distinct color change when the input concentration is higher than the threshold. More than one bit with variable threshold concentrations that are arranged in increasing order can be combined in parallel to achieve transitions between OFF and $\mathrm{ON}$ bits; therefore, the visual read-out can be carried out easily. When the different input concentration is added, the transition between OFF and ON bits will occur at different locations. The ON/OFF idea behind the quantitative assay is also summarized in Figure $7 a^{120}$ In this study, the structure of the SlipChip was constituted of two plates that recessed structures for the aqueous reagents. The pattern of these properties could be used for fluid manipulation. ${ }^{120}$ The benefit of this approach was robustness against temperature and readout time variations. Furthermore, the quantitative instrumentation requirement was eliminated on this platform due to the naked-eye detection. On the contrary, multiple assays could be carried out considering the desired resolution and the dynamic range. Benchmarking the platform performance was carried out with a bead-based sandwich Cys C assay (Figure $7 \mathrm{~b}$ ). ${ }^{120}$ Currently, available threshold chemistries can only detect 210 -fold increases in concentration, and this range cannot be applicable for the Cys C concentrations since it requires detection of less than 1.5-fold for clinical purposes. In this study, Huynh et al. introduced a novel analog-to-digital chemical conversion by creating competitive inhibition, enabling reactions with the substrate to have an increase of 1.22 -fold in theory and 1.30-fold in experiments at nanomolar concentrations that would be adapted potentially with the other assays.

In addition, there have been some hybrid platforms combining microfluidics and NP-integrated platforms for AKI diagnosis. Specifically, a platform was presented to measure the elevation in the expression of certain types of microRNAs (miRNAs), such as miR-494 and miR-16-5p. ${ }^{36}$ These small, single-stranded miRNA molecules show great potential as alternative AKI biomarkers, since they are not present in healthy subjects in contrast to the individuals diagnosed with AKI. ${ }^{36}$ Focusing on these facts, a label-free microfluidic (funnel fluidic) chip integrated with the capped 
a

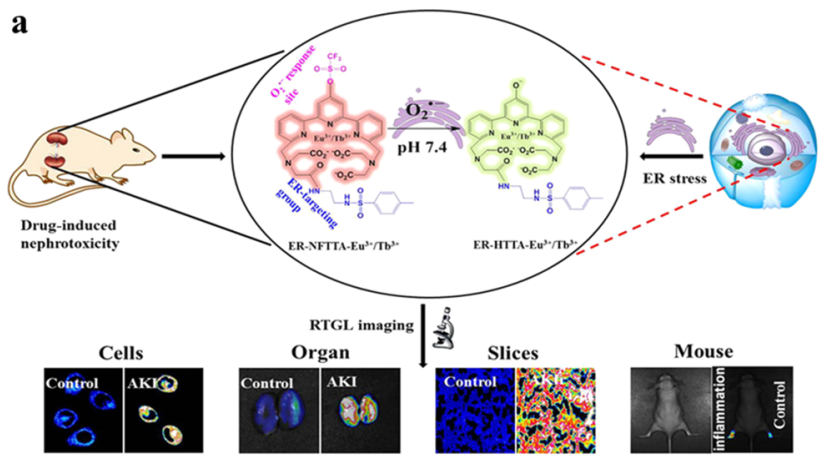

b

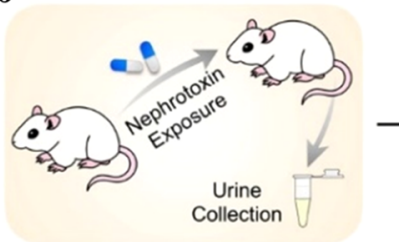

Detection

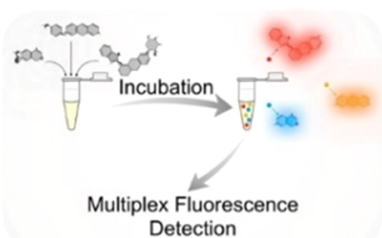

c
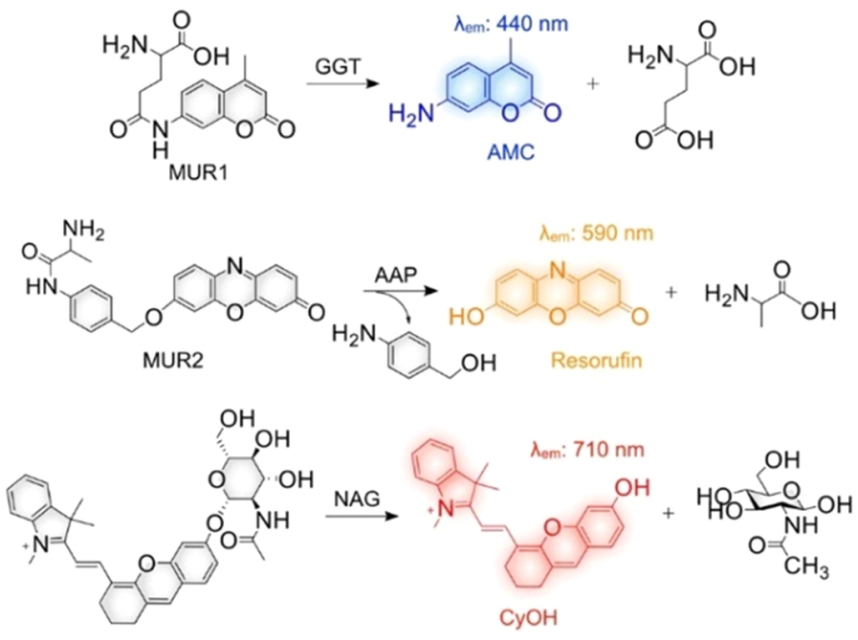

MUR3

Figure 8. (a) Drug-induced AKI is detected in mice kidney through the ER-NFTTA-Eu ${ }^{3+} / \mathrm{Tb}^{3+}$ prob reaction with $\mathrm{O}_{2}{ }^{\bullet-}$ released from ER, and the results are compared with the control group. Reproduced with permission from ref 127. Copyright 2019, American Chemical Society. (b) The application of multiplex fluorescence detection is represented (c) and the structures of MURs and sensing reaction with presence of target urinary AKI biomarkers are shown. Reproduced with permission from ref 129. Copyright 2020, American Chemical Society.

gold nanoslit (CG nanoslit) was developed based on an SPR system for the detection of urinary miRNA-16-5p in relatively large volume samples $(\sim 1 \mathrm{~mL}){ }^{36}$ The idea behind the sensing platform was the isolation of target analytes and detection through CG nanoslit SPR in a microfluidic chip. The structure of the CG nanoslit was fabricated using the nanoimprint lithography on the $178-\mu \mathrm{m}$-thick of cyclic olefin polymer (COP) film. The upper layer contained periodic metallic nanowires, while the lower layer was composed of periodic metallic nanoslits. ${ }^{36}$ The microfluidic device was fabricated with PMMA and double-sided tapes. The isolation and detection of the target molecules were achieved by the double-hybridization technique. As the first step, iron oxide MNPs-functionalized with probe I were used to isolate miRNA-16-5p from urine samples. The SPR surface was then functionalized with probe II, and the target molecule containing MNPs hybridized with probe II. ${ }^{36}$ The velocity of the flow in a microfluidic chip was crucial since the targetcontaining MNPs were able to bind probe II that was immobilized on CG nanoslit. When the flow rate was adjusted to $6 \mu \mathrm{L} / \mathrm{min}$, maximum sensitivity and minimum LOD value were attained. The performance of this sensor platform was evaluated by comparing traditional RNA isolation and quantitative polymerase chain reaction (qPCR) with MNP and RNA isolation from urine. The specificity analysis was determined through mismatch sequences that were distinguished from the fully matched complementary target. Moreover, nonspecific binding was minimized due to the pre-isolation, double hybridization, and optimization of the flow rate. ${ }^{36}$ All these analyses pointed out that the CG nanoslit SPR was able to detect the elevation of miRNA-16-5p; therefore, the double-hybridization method would hold a great potential for clinical applications in AKI diagnosis. However, the requirement of precise control of the flow rate is one impeding factor to be stated for this platform.

\section{MOLECULAR PROBE-BASED DETECTION SYSTEMS}

Molecular imaging has gained notable interest recently for disease detection and identification, the development of personalized medicine for the most efficient therapy, and measurement of molecular-specific effects of treatment by enabling in vivo visualization and quantitative measurement of biological and cellular functions of processes. ${ }^{121}$ In contrast to conventional procedures (e.g., positron emission tomography (PET), single-photon emission computed tomography (SPECT), MRI, magnetic resonance spectroscopy (MRS), ultrasound (US), and CT), molecular imaging provide information on the diseased area without the need for invasive biopsies or surgical procedures. ${ }^{121}$ Optical imaging involves the employment of bioluminescent and fluorescent reporters and probes to interrogate molecular events repeatedly and for monitoring disease progression in animal models. ${ }^{122}$ Activatable chemiluminescent and luminescence probes are also employed for optical imaging. ${ }^{123-125}$ Fluorescent probes have gained substantial attention in disease diagnosis by providing sensitive and rapid procedures and several advantages (e.g., real-time and noninvasive detection/monitoring, high spatiotemporal resolution, and in vivo use) over the years. ${ }^{126}$ For the NAG detection, an enzyme-activated fluorescent probe (NHPO) was developed with high selectivity and sensitivity by using resorufin (HHPO) as a fluorophore. A commercial detection kit and NHPO were compared to confirm NHPO applicability in human urine for the determination of endogenous NAG, and the results emphasized that NHPO indicated high selectivity to human NAG. The use of NHPO was shown to be an effective method for real-time monitoring of the NAG activities. Therefore, the severity of the AKI would also be determined by NHPO strategy. ${ }^{126}$

An ER-targetable luminescence probe (ER-NFTTA-Eu ${ }^{3+}$ / $\mathrm{Tb}^{3+}$ ) was alternatively introduced for early diagnosis of AKI by ratiometric time-gated luminescence (RTGL) monitoring ROS, more particularly, superoxide anion $\left(\mathrm{O}_{2}{ }^{\bullet-}\right)$ that originated from the endoplasmic reticulum (ER), which serves 
a
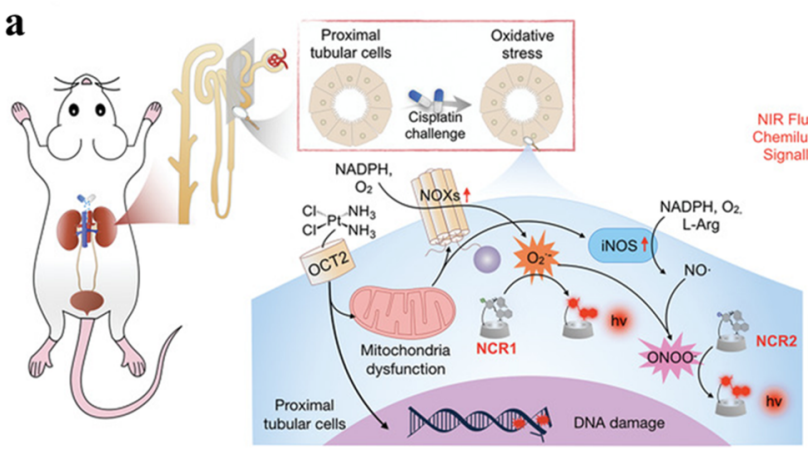

C
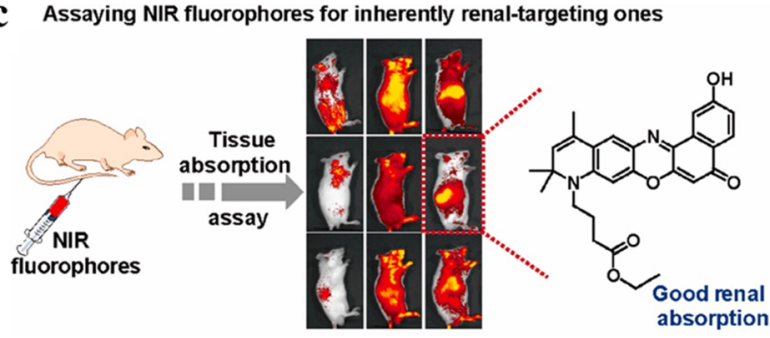

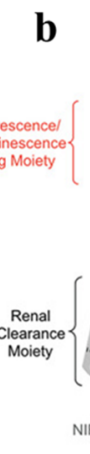

b

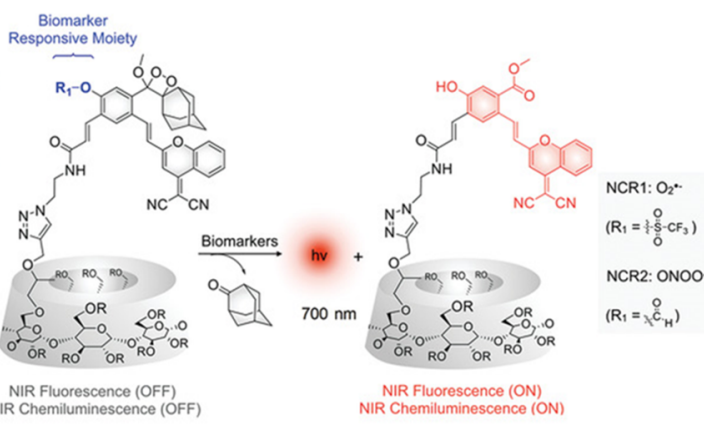

d

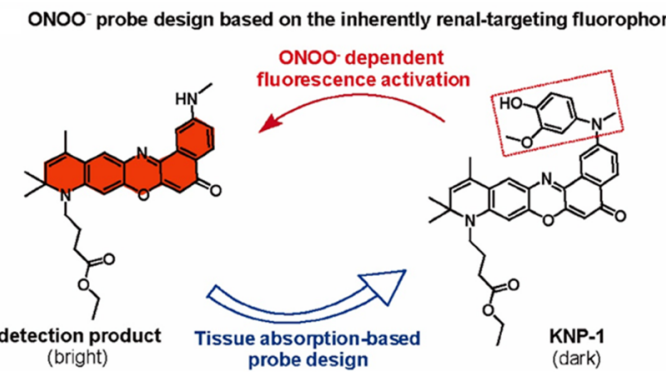

Figure 9. Probe-based diagnosis of AKI. (a) Detection mechanism of probes (NCR1 and NCR2) for $\mathrm{O}_{2}{ }^{-{ }^{-}}$and ONOO is exhibited when AKI condition is present. (b) Chemical structure of NCR1 and NCR2 and their reactions with $\mathrm{O}_{2}{ }^{--}$and ONOO, $\mathrm{R}=\mathrm{H}_{\text {or }} \mathrm{CH}_{2} \mathrm{OHCH}_{3}$ are presented. Reproduced with permission from ref 133 . Copyright $(2020)$ Wiley- $\mathrm{VCH} \mathrm{GmbH}$. (c) The $\mathrm{ONOO}^{-}$detection with kidney-targeting near-infrared fluorophores is represented. (d) Chemical structure of probe and reaction mechanism with $\mathrm{ONOO}^{-}$are illustrated. Reproduced with permission from ref 134. Copyright (2021) Elsevier.

as a precursor of ROS and indicator of AKI. Therefore, it was considered a promising diagnostic biomarker for AKI (Figure 8a). ${ }^{127}$ Several probes have been introduced for $\mathrm{O}_{2}{ }^{\bullet-}$ that have different origins; however, ER has not attracted attention. ERoriginated $\mathrm{O}_{2}^{\bullet-}$ is a crucial point for AKI detection since druginduced AKI leads to ER stress owing to the excessive or unfolded accumulation of proteins in ER. The presented probes indicated a good response in the presence of $\mathrm{O}_{2}{ }^{--}$. In addition, it displayed good water solubility, high sensitivity and selectivity, low cytotoxicity, and excellent ER-localization. ${ }^{127}$

In addition to these studies for the detection of NAG and superoxide anion $\left(\mathrm{O}_{2}{ }^{\bullet-}\right)$, a recent work combined these two indications by synthesizing activatable duplex reporter (ADR)-also called a dual-channel optical probe. ${ }^{128}$ Here, the ADR provided chemiluminescence and near-infrared fluorescence (NIRF) signals in the presence of oxidative stress $\left(\mathrm{O}_{2}{ }^{\bullet-}\right)$ and lysosomal damage (NAG), respectively. When the $\mathrm{ADR}$ was administrated to the living mice system, kidney injury-related two molecular events (i.e., oxidative stress and lysosomal damage) were imaged in the form of chemiluminescent $\left(\mathrm{O}_{2}{ }^{--}\right)$and NIRF (NAG) signals. As a result of this imaging study, GFR decline and renal tissue damage were able to be determined earlier compared to the existing clinical assays. Overall, the ADR-based platform offered real-time, noninvasive, and simultaneous monitoring of two correlated biomarker levels, as well as eliminated the signal cross-talk. The renal clearance efficiency of the ADR was observed as $80 \%$ of injected doses after $24 \mathrm{~h}$ injection, and this ADR detected AKI at least $8 \mathrm{~h}$ (NIRF) and up to $16 \mathrm{~h}$ (chemiluminescence) earlier, accelerating intervention options for AKI treatement. $^{128}$

In addition, the probe-based detection system have enabled the multiplex detection of AKI biomarkers to increase diagnostic capability. Molecular urinary reporters (MURs) were introduced for the early diagnosis of drug-induced kidney injury (DIKI). The idea behind the MURs was to introduce activatable reporters capable of detecting multiple urinary AKI biomarkers. The biomarkers used to design MURs were GGT, alanine aminopeptidase (AAP), and NAG, which are elevated in urine due to glomerulonephritis and ATN at the early stages (Figure $8 \mathrm{~b}$ ). ${ }^{129}$ AAP and GGT are the enzymes (e.g., proximal tubule brush border enzymes) that are released from the damaged tubular cells; however, NAG is the hydrolytic lysosomal enzyme originating from the lysosome of the proximal tubule cell. ${ }^{21,22,129,130}$ When the tubules are injured, enzymes are released and secreted to urine. If the measurement of the enzymes is performed, the size, site, or nature of the damage in tubular cells and their possible necrosis or dysfunction can be designated. ${ }^{131} \mathrm{AAP}$ is one of the promising AKI biomarkers due to being a brush border enzyme and is very sensitive to renal tubular damage. When damage occurs in the brush border, microvilli are lost, and the AAP level is elevated in the urine because of secretion. This elevation is proportional to the increase of $\mathrm{SrC}$ and decrease of GFR. The sensitivity of the enzymes is higher than that of the secreted proteins, since enzyme increase occurs before protein increases. The increase of the APP can be observed at very early stages (e.g., the first day of injury). ${ }^{130}$ Each mechanism and color of the MURs is specific to a biomarker. MUR1(Lglutamic acid $\gamma$-(7-amido4-methylcoumarin) is specific to GGT, and after enzymatic cleavage, it emits blue fluorescence signals. MUR2 is a resorufin-derived orange-fluorescent dye for detection of AAP from cleavable alanine amino acid by a selfimmolative linker (Figure $8 \mathrm{c}$ ). MUR3 is obtained by caging a NAG-cleavable responsive group of NIR fluorescent hemicyanine dye. Therefore, the color of the MUR 2-3 is observed in the presence of the target biomarker. ${ }^{129}$ 
a
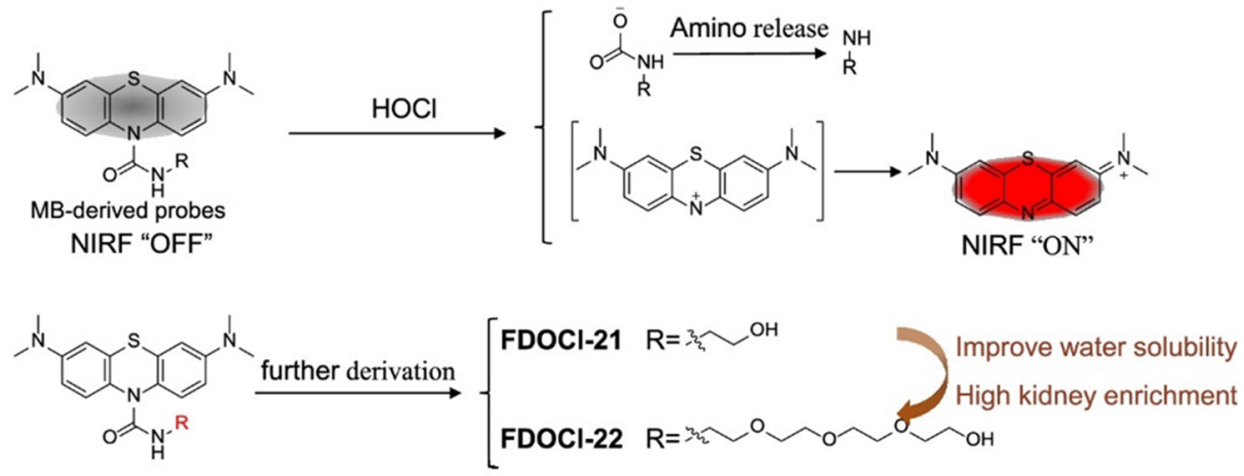

b

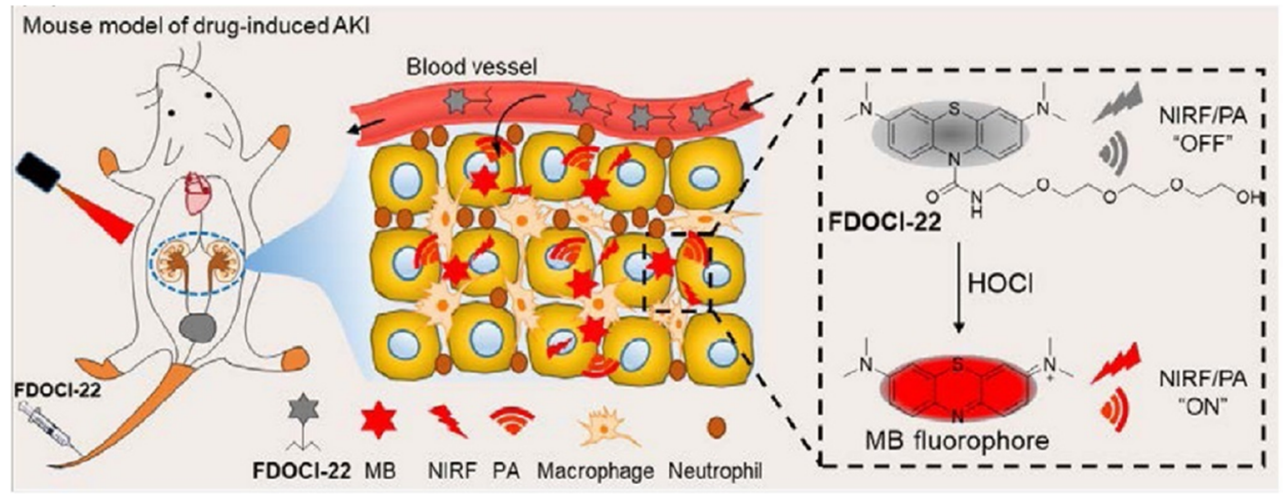

Figure 10. Probe-based diagnosis. (a) $\mathrm{HOCl}$ activatable FDOCI-22 probe structure and water-soluble probe along with the modification are presented. (b) A schematic represents the dual-modality detection of drug-induced AKI at an early stage. Reproduced from ref 135 . Copyright (2020) American Chemical Society.

Employing optical imaging techniques is a promising strategy due to high spatiotemporal resolution, superb sensitivity, and the ability to measure slight changes of biomarker level corresponding with the disease region. ${ }^{132}$ Conventionally, kidney imaging is carried out via SPECT, contrast-enhanced computed tomography, MRI, and ultrasonography; however, their performance is not sufficient for the determination of early stages of AKI at the molecular level. ${ }^{132}$ Recently, optical (renal-clearable) molecular renal probes (MRPs) were developed to visualize sequential prodromal molecular events such as early-stage biomarkers of AKI in real-time from murine models, suffering from druginduced AKI. In this study, the probes were designed through a renal clearance moiety, a biomarker reactive moiety, and a luminescent signaling moiety. The target AKI biomarkers were $\mathrm{O}_{2}{ }^{\bullet-}$, NAG, and caspase-3, since they provide information about oxidative stress, lysosomal damage, and cellular apoptosis, respectively. ${ }^{132}$ In the presence of target biomarkers, near-infrared fluorescence (NIRF) was obtained from MRPs, which were single-channel probes. Another MRP $\left(\mathrm{MRP}_{\mathrm{D}}\right.$ : dual channel probe) was also investigated. This probe was dualchannel, and so it became chemiluminescent when it underwent a reaction with $\mathrm{O}_{2}{ }^{--}$, and the reason for the second $\mathrm{MRP}$ for $\mathrm{O}_{2}{ }^{--}$was simultaneous monitoring; therefore, upregulation of $\mathrm{O}_{2}{ }^{\circ-}$ and GFR change comparison would be carried out directly. AKI detection via MRP1-2 and $M R P_{D}$ was achieved before the depletion in GFR; a more specific timeline of the MRPs1-3 AKI detection was 12, 16, and $48 \mathrm{~h}$, when cisplatin-induced AKI developed. ${ }^{132}$ In clinical diagnosis, MRPs are promising, since they enable rapid renal clearness and nontoxicity. As a consequence, molecular probes were introduced as imaging agents by reacting specifically with biomarkers to identify abnormal upregulation before GFR decrease via spontaneously activated optical signals. ${ }^{132}$

On the other hand, a recent study introduced near-infrared (NIR) chemiluminescent reporters (NCRs) to image reactive oxygen and nitrogen species (RONS) for AKI diagnosis in mice models since they had high renal clearance (Figure 9a). ${ }^{133}$ The structure of the NCRs composed of (2hydroxypropyl)- $\beta$-cyclodextrin ( $\mathrm{HP} \beta \mathrm{CD}$ ), enabling renalclearance, and modified dicyanomethylene- $4 \mathrm{H}$-pyran containing Schaap's dioxetane as a chemiluminescent moiety (Figure $9 \mathrm{~b})$. The selected biomarkers were superoxide anion $\left(\mathrm{O}_{2}{ }^{--}\right)$ and peroxynitrite $\left(\mathrm{ONOO}^{-}\right)$, since oxidative and nitrosative stresses are the main indicators of drug-induced AKI. The working mechanism of these NCRs is to react with RONS biomarkers once they reach the kidneys; accordingly, oxidative/nitrosative stresses can be reported. These reporters determine not only subtle upregulation RONS, but also allow noninvasive monitoring. ${ }^{133}$ In addition, peroxynitrite $\left(\mathrm{ONOO}^{-}\right)$can be employed alone via near-infrared fluorophore (KNP-1) to image AKI, since it is upregulated when the AKI condition is developed and also associated with the severity of the disease. ${ }^{134}$ Moreover, the upregulation of $\mathrm{ONOO}^{-}$occurs $24 \mathrm{~h}$ earlier compared to $\mathrm{sCr}$ and $\mathrm{BUN}$. $\mathrm{ONOO}^{-}$has the ability to react with the lipids, DNA, and proteins, directly or indirectly, and this reaction causes cellular injury, inflammation, and apoptosis, ending up with AKI. In this work, the mechanism was started with the administration of the KNP-1 intravenously, and then it was activated by the $\mathrm{ONOO}^{-}$, and as a result, a good contrast imaging for kidneys was achieved (Figure 9c). The diagnostic capability of the KNP-1 is valid for both nephrotoxin-induced and ischemiareperfusion injury-induced AKI in mice models. ${ }^{134}$ These two 
Table 2. Evaluation of the Commercially Available Products for Acute Kidney Injury

\begin{tabular}{|c|c|c|c|c|c|c|}
\hline name & company & country & approval & target marker & dynamic range & refs \\
\hline RENISCHEM & $\begin{array}{l}\text { CMIC } \\
\text { HOLDINGS } \\
\text { Co. }\end{array}$ & Japan & CE-marked & L-FABP & Not reported & 154 \\
\hline Nephrocheck & Astute Medical & $\begin{array}{l}\text { Italy, Austria, Germany, France, Switzerland, } \\
\text { the UK, and Spain }\end{array}$ & $\begin{array}{l}\text { CE-marked } \\
\text { FDA }\end{array}$ & $\begin{array}{l}\text { TIMP-2/ } \\
\text { IGFBP7 }\end{array}$ & Not reported & $87,156,158$ \\
\hline $\begin{array}{l}\text { Rena-Strip Lateral- } \\
\text { Flow Kit }\end{array}$ & BioAssay Works & Not specified & $\begin{array}{l}\text { No } \\
\text { information }\end{array}$ & KIM-1 & $\begin{array}{l}4 \mathrm{pg} / \mathrm{mL} \text { to } 40,000 \\
\mathrm{pg} / \mathrm{mL}\end{array}$ & 148,159 \\
\hline
\end{tabular}

studies emphasized that $\mathrm{ONOO}^{-}$is a promising target for early diagnosis of AKI. ${ }^{133,134}$ Besides these biomarkers, a hypochlorous acid ( $\mathrm{HOCl})$ activatable probe (FDOCl-22) for dualmodality detection of drug-induced AKI at an early stage was also reported. ${ }^{135}$ This probe was dissolvable in water (Figure 10a) and exhibited high sensitivity against $\mathrm{HOCl}$ (Figure 10a). When FDOCl-22 reacted with $\mathrm{HOCl}$, notable elevation was observed in both near-infrared (NIR) emission and absorption; therefore, $\mathrm{HOCl}$ level was associated with the druginduced AKI (Figure 10b). Furthermore, using fluorescence in NIR range and photoacoustic imaging enabled early stage drug-induced AKI detection with this probe. ${ }^{135}$

\section{OTHER DETECTION STRATEGIES}

Biosensing strategies are primarily reliant on the capture of target analytes through a receptor, and consequently, the signals obtained from these biochemical interactions are translated into a readable form through a transducer. In addition, recent advancements in science and technology have brought new insights for the detection strategies. Target recycling amplification, for instance, has attracted substantial interest, since it offers excellent efficiency and superb selectivity. Besides these advantages, this approach also has limitations, such as enzymes requiring specific conditions to stay functional. ${ }^{136}$ Moreover, restriction endonucleases are widely employed, and they need specific nucleotide sequences for the recognition. ${ }^{137}$ Different signal amplification strategies, such as DNA-based signal amplification including polymeraserelated signal amplification, exonuclease signal amplification, and nicking endonuclease signal amplification, are present; however, these methods require enzymes, which bring some limitations as mentioned above. ${ }^{138}$ To minimize these drawbacks as much as possible, enzyme-free recycling amplification strategies have been developed. ${ }^{136}$ Moreover, these strategies can also be integrated into a biosensing approach such as electrochemical ${ }^{137}$ or SERS ${ }^{139}$ instead of using bulky instruments (e.g., spectrophotometer ${ }^{140}$ ) to diagnose diseases such as cancer $^{137}$ or kidney diseases. ${ }^{141}$ For instance, this DNase-I-assisted recycling signal amplification strategy was applied for the determination of AKI at an early stage by targeting NGAL biomarker. ${ }^{142}$ A nanocomplex combining the polydopamine nanosphere (PDANS) with a fluorophore (5-carboxyfluorescein (FAM))-labeled aptamer was utilized for NGAL determination. ${ }^{142}$ The PDANS/ aptamer formed a nanocomplex owing to $\pi-\pi$ stacking between aptamer and PDANS. The formation of the nanocomplex created fluorescence quenching via the Förster resonance energy transfer (FRET) principle. The NGAL binding to aptamer created an increase in the fluorescence intensity, and the detection range was between 12.5 and 400 $\mathrm{pg} / \mathrm{mL}$, along with $6.25 \mathrm{pg} / \mathrm{mL}$ of an LOD value; ${ }^{142}$ however, the main disadvantage of this strategy was the involvement of enzymes, which require specific storage conditions.
Moreover, a similar principle was adapted to determine renal interstitial fibrosis (RIF), an inevitable pathological pathway and reliable predictor of $\mathrm{CKD}$ prognosis-associated with AKI. ${ }^{141,143}$ For monitoring the progression of RIF, dual markers (matrix metalloproteinase-9 (MMP-9), and matrix metalloproteinase-2 (MMP-2)) were quantified by utilizing DNase-I-assisted recycling signal amplification with the polydopamine nanosphere and fluorescence-labeled aptamers, and similarly, this strategy utilized FRET modality. ${ }^{141}$ FAM and Texas Red were used for labeling aptamers to create fluorescence signals. The fluorescent measurements were carried out at 520 and $610 \mathrm{~nm}$ for MMP-9 and MMP-2, respectively. The LOD values were 9.6 and $25.6 \mathrm{pg} / \mathrm{mL}$ for MMP-9 and MMP-2, respectively. ${ }^{141}$

As aforementioned, different miRNA types (e.g., miR-494 and miR-16-5p) have been utilized in AKI diagnosis. ${ }^{36}$ For instance, miRNA-21 and miRNA-200c are also associated with the drug-induced AKI, and accordingly, novel hairpin DNAfueled nanoflares have been introduced to determine their levels by using target catalytic recycling amplification. ${ }^{144}$ Briefly, two hairpin DNA ( $\mathrm{H} 1$ for miR-21 and $\mathrm{H} 2$ for miR200c) were labeled with FAM and cyanine 5 (Cy5), and they were immobilized on the surface of AuNPs. The strategy was to hybridize the double strands, including H1-miR-21 and H2miR-200c, when miRNA was encountered with nanoflares, and eventually, these interactions resulted in two-colored fluorescence signals. The LOD values of these strategies were 18.1 and $21.1 \mathrm{pM}$ for miRNA-21 and miRNA-200c, respectively. ${ }^{144}$

In another study determining the levels of miRNA-21, a personal glucose meter (PGM) was developed. ${ }^{145}$ The platform constituted streptavidin-immobilized magnetic beads (MBs)-coated with RNA-cleaving DNAzyme strands through biotin-avidin interactions. RNA-cleaving DNAzyme strands were silenced by a locking strand and substrate strands connected to the invertase (Inv). With these conjugations, MBs-DNA-Inv complexes were formed. The main principle of this platform was to convert miRNA-21 into amplified glucose signals through the interactions between DNAzyme and Inv. The procedure for this conversion was the hybridization of miRNA-21 with locking strand through a strand-displacement reaction. After this reaction, the locking strand was released from the DNAzyme that a cofactor $\mathrm{Mn}^{2+}$ activated to cleave substrate strands from the MB surface. Following that, glucose conversion was achieved by magnetic separation, the transfer of Inv to the sucrose solution, which catalyzed saccharose into glucose. At the end of the reaction, a small amount of miRNA21 was converted to glucose and quantified by PGM. The linear dynamic range was observed between $100 \mathrm{fM}$ and $1 \mathrm{pM}$, and the LOD value was found as $68.08 \mathrm{fM} .^{145}$

Consequently, all these detection strategies have revealed new insight in AKI diagnosis along with a variety of advantages and limitations. As a highlighting example, miRNA-21 detection with PGM has provided a notable enhancement in 
a

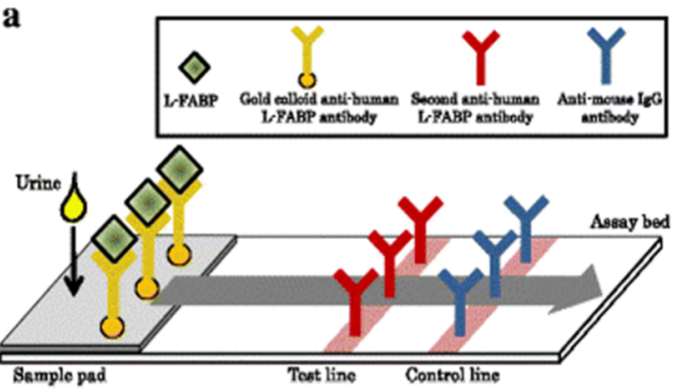

b

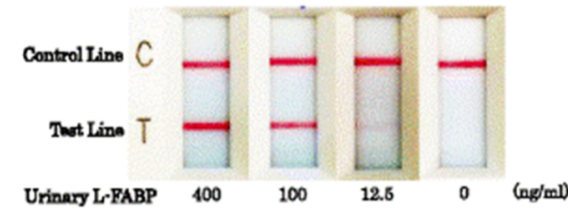

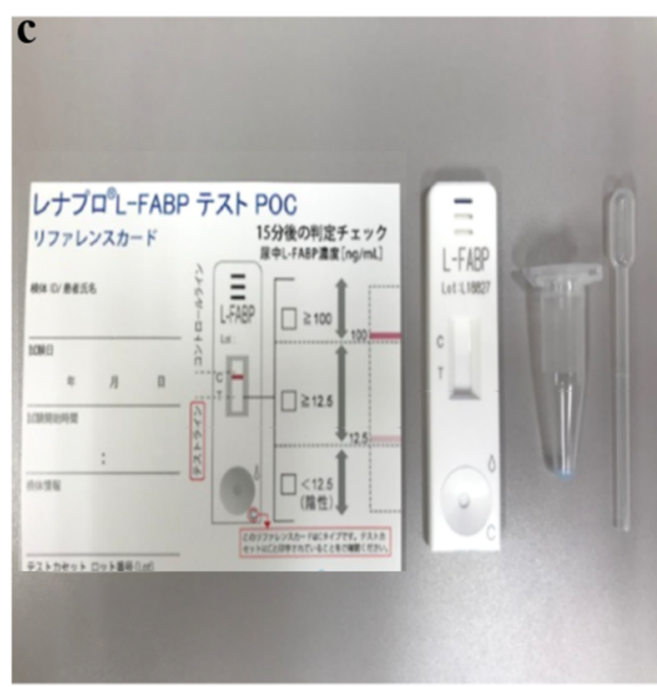

Figure 11. (a) The working principle of POC assay kit. (b) Samples in different concentrations of urinary L-FABP are evaluated. Reproduced with permission from ref 146. Copyright 2017, Springer Nature. (c) Commercial product of this assay is represented. Reproduced with permission from ref 155. Copyright 2019, Springer Nature.

specificity and discrimination to single-base mutation. ${ }^{145}$ Signal enhancements also increased the sensitivity; however, involvement of enzymes limits their broad utility at remote settings, where there is a lack of sufficient storage conditions. ${ }^{145}$

\section{COMMERCIALLY AVAILABLE DIAGNOSTICS}

Advanced technologies offer huge opportunities for clinical diagnoses, since they enable the development of easy, rapid, and cost-efficient detection tools. In this regard, the improvements in AKI diagnosis have been conducted with biosensing strategies focusing on common (e.g., increase in Crea level, BUN, or urine output) and novel biomarkers (e.g., KIM-1, NGAL, L-FABP, and TIMP-2/IGFBP7). Later on, some of these biosensing platforms such as RENISCHEM, Nephrocheck, and Rena-Strip Lateral-Flow Kit have been commercialized to determine specific AKI biomarkers. ${ }^{146-148}$ Their properties are briefly summarized in Table 2 . The enhancement in the diagnostic capability of AKI is crucial, since the late diagnosis of AKI leads to the development of chronic kidney disease (CKD) or even progression to endstage renal disease (ESRD) and cardiovascular diseases. ${ }^{149-151}$ The main treatment of the ESRD is renal transplantation, which brings different kinds of risks, such as renal graft rejection, which can also be determined through integrating kidney exosome analysis (iKEA). ${ }^{152}$

RENISCHEM: L-FABP POC Kit. The POC test kit is approved in Japan, and produced by the CMIC HOLDINGS Co., Ltd., Tokyo, Japan. ${ }^{146,153}$ This kit basically focuses on urinary L-FABP. Two categories of patients (patients in ICU with sepsis and outpatients with CKD) were enrolled in performing POC assay while ELISA was performed as a gold standard method to compare and ensure consistency of the results. ${ }^{146}$ In the kit, there are multiple items, such as the inset kit components, summary, and explanation of the test, along with some information like the principle of the kit, the warning and precautions, storage conditions, stability, specimen collection, test, assessment procedure, test results, clinical implications, and performance characteristics. ${ }^{154}$ The intended use is specified as the capability to detect and semiquantitatively determine urinary L-FABP from humans. The purpose of the kit is to diagnose renal disease with renal tubule dysfunction. ${ }^{154}$ The explanation of the test is based on the LFABP mechanism, which has a significant role in energy and lipid metabolism by solubilizing and reabsorbing due to its low molecular weight in the proximal tubule. Ischemic and oxidative stresses lead to L-FABP secretion into urine, which is the response to proteinuria. ${ }^{154}$

Urinary L-FABP is a biomarker for AKI, which is majorly caused by open-heart surgery and sepsis. However, the detection procedure of the urinary L-FABP requires more than a few days, so the evaluation of the AKI progression and severity becomes more complex. Therefore, a rapid detection method would be beneficial. ${ }^{155}$ A recent study has introduced a new measurement assay that has the ability to measure LFABP from urine rapidly, and this assay is based on immunechromatography, providing results within $15 \mathrm{~min}$. The working principle is summarized in Figure 11. Briefly, when the urine sample was applied to the sample pad, L-FABP proteins were attached to colloidal gold, which was conjugated with antihuman L-FABP antibodies. Thanks to the capillary effect in the assay pad, the antigen-antibody complexes moved to a second anti-human L-FABP antibody located on the test line and provided a red-line. ${ }^{146}$ The detection was based on a semiquantitative measurement by comparing the color intensity. As a control, anti-mouse IgG polyclonal antibody was used. After binding, burgundy color was observed. ${ }^{154}$ The assessment of the results was based on the comparison of color intensity with the reference card, which was provided by the kit. $^{154}$

Nephrocheck. Nephrocheck is a commercially available test kit that has the ability to measure a combination of TIMP2/IGFBP7 from urine to discriminate an AKI condition from non-AKI. ${ }^{147}$ It was approved by the FDA on September 5, 2014, on behalf of Astute Medical. ${ }^{87}$ The principle of the kit is based on the quantitative, fluorescent sandwich immunoassay. Here, the collected urine samples require centrifugation and mixing with a test buffer.

In a study, the device consisted of the test buffer and conjugate vials, which contained monoclonal and polyclonal antibodies against TIMP-2 and IGFBP-7, fluorescent dye, stabilizers, and excipients. ${ }^{156}$ The intended use of the kit is 
determined in the manual, mainly specifying the target group to be applied and the purpose of this assay. The use of this kit is limited for the ICU patients, who are older than 21 years old and who may have cardiovascular and/or respiratory failure or had at least one of them within the past $24 \mathrm{~h}$. They also firmly state that the purpose is "an aid in the risk assessment for moderate or severe acute kidney injury (AKI) within $12 \mathrm{~h}$ of patient assessment". 157

Discovery, Sapphire, Opal, and Topaz studies were conducted for detection and validation of TIMP-2/IGFBP7 in the prediagnosis of AKI before the marketing of Nephrocheck, since the FDA did not approve any plasma or urine biomarkers to assess the risk of AKI or diagnose. ${ }^{87,147} \mathrm{~A}$ Discovery study was performed to identify novel biomarkers; therefore, 340 possible biomarkers (e.g., NGAL, KIM-1, IL-18, and L-FABP) were examined from urine to evaluate risk assessments for AKI in 522 individuals. The individuals were selected in ICU with sepsis or only one risk factor. The results demonstrated that TIMP-2/IGFBP7 had the best performance (AUROCs of 0.75 and 0.77 , respectively). ${ }^{87}$ Sometimes, the Discovery phase is included in the Sapphire study with the validation phase; however, the results and parameters are the same. ${ }^{147}$ A Sapphire study was carried out for the multicenter validation by enrolling 728 critically ill adults, who had respiratory and/or cardiovascular impairments. The cohort was selected from the individuals, who were admitted to the ICU within $24 \mathrm{~h}$. Stage 2 or $3 \mathrm{AKI}$, according to KDIGO, is not included in the study. ${ }^{87}$ The results showed that TIMP-2/ IGFBP7 provided better performance, similar to the Discovery study. More importantly, the level of TIMP-2/IGFBP7 did not increase in the CKD condition. ${ }^{87}$ Based on the Sapphire study results, the accuracy and clinical utility confirmation of two cutoff values ( 0.3 and 2.0 ) were applied by the Opal study, in which 154 critically ill adults were enrolled for the validation. The individuals in the cohort study were older than 21 years old and in an ICU. They commonly had respiratory or CV impairments and indwelling Foley catheters. ${ }^{87}$ Topaz mostly referred to clinical adjudication of the primary end point to validate. It was applied to 420 critically ill patients in the ICU and older than 21 years, who had respiratory or $\mathrm{CV}$ impairment, indwelling Foley catheters, and $83 \%$ exposed nephrotoxic medication. TIMP-2/IGFBP7 showed persistent results and remained crucial even in clinical studies. ${ }^{87}$ The biomarker was not approved for the use of individuals, who are younger than 21 years, since the level of the TIMP-2/IGFBP7 was decreased in pediatric cases, who underwent $\mathrm{CPB}$ surgery after $4 \mathrm{~h}$ because of unknown reasons. ${ }^{87}$

According to the data obtained from these studies, which cover more than 1800 heterogeneous critically ill patients, it can be stated that urinary TIMP-2/IGFBP7 holds enormous potential for the early detection and risk assessment for AKI. ${ }^{147}$ In addition to FDA, NephroCheck has been CE-marked (Conformité Européenne), so it can be found in some European countries, such as Italy, Austria, Germany, France, Switzerland, the UK, and Spain. ${ }^{158}$

Rena-Strip Lateral-Flow Kit. The investigation of the KIM-1 has enabled new measurements and provided several advantages compared to conventional ELISA. The most crucial benefit is eliminating the dilution of urine samples to measure KIM-1 protein, since it has an extended dynamic range, such as from $4 \mathrm{pg} / \mathrm{mL}$ to $40,000 \mathrm{pg} / \mathrm{mL}$. Additionally, the required volume of urine sample could be decreased down to $30 \mu \mathrm{L}$ (undiluted) within a shorter time (reduction from 6 to $3.5 \mathrm{~h}$ ) for the quantification. During the procedure, inter- and intraassay variability between KIM-1 values is preserved at $<10 \%$. The assay relies on a microbead-based strategy, focusing on the pair of epitopically distinct mouse monoclonal antibodies against the rat KIM-1 ectodomain. ${ }^{159}$ In addition, another immunoassay provided the result within $15 \mathrm{~min}$. The developed tool was reliant on a lateral-flow dipstick immunoassay, called Rena-Stick, which was able to quantify urinary KIM-1 from mice. The development procedure of the tool was started with a combination of the mouse anti-KIM-1 capture antibody and gold nanoparticles. The next step was embedding on a polyester ribbon. Nitrocellulose membranes were used for stripping the detection antibody and controlling the IgG antibody with a distance of $1 \mu \mathrm{L} / \mathrm{cm}^{2}$ from each other. The ribbon and the membrane were assembled together and placed into the immunochromatographic housing. ${ }^{180}$ This approach was implemented by BioAssay Works and assays for KIM-1currently on sale. The KIM-1 Detection Assays contain both traditional ELISA tests and Rena Lateral-Flow kits, and one can use either human or rat urine samples. Although Rena Lateral-Flow kits provide rapid and easy-to-use fashions, they present semiquantitative measurements. ${ }^{148}$

A strip form of the earlier kit (Rena-Strip Lateral-Flow Kit) is also developed, and it provides results within $15 \mathrm{~min}$ from human urine and $25 \mathrm{~min}$ from rats. The procedure is easily applicable (starting with the application of a sample, incubation, and recording the results) and cost-effective since it does not require any additional reagents or special equipment. The detection of KIM-1 is basically carried out semiquantitatively, so an assessment is performed by comparison of a given color chart or reading with a lateralflow assay reader. ${ }^{48}$ The strip is composed of antibody-gold conjugates, which create a complex with KIM-1 when the contact flowing samples and conjugate occur. The formed complex then moves through the strip and reaches to a nitrocellulose membrane, which contains an immobilized antiKIM-1 antibody. The red-line reflects the presence of KIM$1 . .^{148}$

\section{RENAL TRANSPLANT DETECTION METHOD}

AKI has been considered a completely treatable and reversible disease for a long time; however, if it is not treated as early as possible, it will progress into CKD or ESRD. ${ }^{151,161}$ Even if the patients survive from AKI, there is a high risk of development of CKD or even progression to ESRD and cardiovascular diseases. ${ }^{149-151}$ After the progression of the ESRD, kidney transplant is the definite treatment procedure; however, longterm allograft rejection is the main adverse effect. Renal graft rejection is determined by biopsy as a gold standard, yet leads to several disadvantages, including inherent procedural risk, complications, and relatively high costs. To overcome from such issues, a novel detection platform was introduced to determine kidney transplant rejection from urine, targeting to T-cell-specific extracellular vesicles (EVs) by iKEA. ${ }^{152}$ The idea behind the platform was infiltration of the $T$ cells to kidney tubules when the acute cellular rejection (ACR) of kidney allografts developed. Therefore, they were gaining close proximity for urination. As a consequence, the presence of $\mathrm{T}$ cell-induced EVs in urine has risen possibly, and their determination would indicate ACR. ${ }^{152}$ The built-up platform detects urinary EVs from T-cell lymphocytes based on the magneto-electrochemical strategy (a combination of magnetic selection with electrochemical detection). CD3 was detected as 
a potent marker (e.g., diagnostic accuracy of $91.1 \%$ and $83.7 \%$ in a discovery and validation cohort) for the T-cell-derived urinary EVs when the iKEA was applied to cell culture and clinical urine samples. ${ }^{152}$ The detection procedure was basically composed of (i) EVs enriched via immunomagnetic capture, (ii) collecting and labeling EVs, (iii) generation of electrical current through mixing beads with a chromogenic electron mediator, and (iv) combination of an iKEA device to read-out electrochemistry. By using this procedure, high sensitivity was achieved. This portable detection system was composed of three electrodes (working (W), counter (C), and reference $(R))$ for the measurements. The electrical current between $\mathrm{W}$ and $\mathrm{C}$ electrodes was measured by a customdesigned potentiostat, and $\mathrm{R}$ and $\mathrm{W}$ electrodes were exposed to a constant potential simultaneously. ${ }^{152}$ The comparison with conventional detection systems such as ELISA and Western blotting demonstrated that iKEA's sensitivity and rapidity were higher. As a consequence, in this study, iKEA offered noninvasive, serial monitoring to achieve the improved outcomes and also eliminated the complications after renal transplantation. ${ }^{152}$

\section{CONCLUSION}

AKI is a clinical syndrome diagnosed with the accumulation of nitrogen metabolism (e.g., creatinine and urea) and the decrease of GFR with urine output reduction within a short period, like hours or days. Over the past years, $\mathrm{SrC}, \mathrm{BUN}$, and UO levels, which are traditional biomarkers, have been employed to characterize GFR. Briefly, conventional diagnostic markers provide information about the excretory function of the renal system only; however, endocrine or immunological function of kidneys needs to be evaluated as well. SrC, BUN, and UO are not adequately accurate, since they can be influenced by the other systems or organs. ${ }^{12}$ Regarding these facts, the decrease of GFR is not sensitive enough, and this also leads to the late indications of AKI, as most injuries take place in the tubular epithelial cells instead of the glomerulus. On the other hand, the main reason for the increased mortality and morbidity due to AKI development is deficiencies in effective treatment and diagnostic procedures. However, there should be a timeline to overcome these limitations, because effective treatment cannot be applied without a definite diagnostic procedure. To address these limitations, two different approaches are utilized: (i) seeking novel and more sensitive biomarkers for AKI and (ii) developing selective, sensitive, affordable, portable, and easily accessible diagnostic platforms. Therefore, in this review, we comprehensively benchmark both interrelated concepts, i.e, biomarkers and biosensing strategies/assays, on the same page. These biomarkers can be constitutive proteins and elevated molecules (e.g., proteins, encapsulated molecules in exosomes, miRNA, and siRNA) as a response to injury. ${ }^{11}$ The discovery of novel biomarkers does not enable enhancing the diagnostic procedure of AKI alone, since currently employed quantification assays and methods have crucial drawbacks for clinical application. These methodologies have not been standardized, and they are mostly timeconsuming, so discovering novel and accurate biomarkers is not enough to enhance the impact on the technology site of AKI diagnosis. Hence, rapid, easy-to-use, facile measurement and affordable detection tools are required for further applications. Traditionally, diagnosing the diseases is carried out in the clinical laboratories with several substantial requirements, such as highly qualified personnel to collect samples, employing the test procedure, and evaluating or interpreting the results; and the utilization of sophisticated facilities equipped with full-fledged devices. In this regard, several ongoing research studies are promising to encounter these needs. Considering all these parameters, the main approach to satisfy these features is biosensing platforms.

In this review, we have presented a variety of biosensing platforms for AKI diagnosis targeting different biomarkers (SrC, NGAL, KIM-1, Cys C, miRNA) through different capturing elements (both natural or biomimetic elements). The platforms presented here have been further improved with nanomaterials, such as the addition of graphene layer or the integration of gold nanoparticle. For the advancement of the diagnostic capability, multiplexing detection of more than one biomarker has been also achieved to broaden the causes of AKI development in the body. There are also commercially available assays/platforms for AKI diagnosis, such as RENISCHEM that detects urinary L-FABP; Nephrocheck that detects urinary TIMP-2/IGFBP7; and Rena-Strip LateralFlow Kit that detects KIM-1 protein from urine. However, these products are not accurate for all individuals. They are mostly limited to some criteria, since different etiologies (age, ${ }^{162}$ gender, race, diabetes mellitus, ${ }^{163}$ major surgery, ${ }^{164}$ chronic kidney disease, sepsis, ${ }^{165}$ and hypovolemia ${ }^{166}$ ) of AKI cause different outcomes. For instance, RENISCHEM can be applied for patients in ICU with sepsis and outpatients with CKD. The application of Nephrocheck is not allowed for the use of individuals, who are younger than 21 years. In addition, the principle of RENISCHEM relies on the lateral flow assay, which does not provide quantitative results, and also, RenaStrip Lateral-Flow Kit determines KIM-1 levels semiquantitatively by benefiting from a color chart. On the other hand, as an example, SPRi-based nanoimmunosensor offered label-free, real-time measurement and multiplexed detection with quantitative results, which are crucial in detecting biomarkers. ${ }^{83}$ Through this platform, the level of the KIM-1 was conducted to indicate the severity of AKI. ${ }^{28}$ For the NGAL detection, molecular imprinting gold nanocages have been developed, ${ }^{85}$ and they could also be adapted for the detection of KIM-1 and the other target molecules. MIPs are introduced for recognition as an alternative to antibodies, and they are synthetic polymers that can capture target molecules, whose surface signatures at molecular levels have been templated on a polymeric matrix. In addition to MIPs, affinity peptides were also employed on an electrochemical biosensor for NGAL detection, but their synthesis was complicated and time-consuming. ${ }^{56}$ The main advantage of these platforms was more robust and stable detection with a label-free strategy. Moreover, biosensors also enable a dual detection strategy, for instance, the combination of SERS and DPV for Cys C. ${ }^{89}$ Nephrocheck detects TIMP-2/IGFBP7 with a fluorescent dye; however, an SPRi-based nanoimmunosensor does not require any labeling procedure in detecting both KIM-1 and IGFBP7. ${ }^{83}$ RENISCHEM provides semiquantitative results and requires antibodies labeled with gold nanoparticles for detection; on the other hand, such biosensors like SPRi offer quantitative detection and provide a label-free strategy, thereby reducing the assay utility, testing cost, and process steps significantly.

In addition to detecting or quantifying the target biomolecule on a biosensor, molecular probes that target the indicators of AKI are also detailed in this review. These strategies enable real-time monitoring and multiplex detection, 
but there are some limitations. For instance, some of these systems are invasive, and they would provide high renal clearance. Furthermore, target recycling amplification strategies are utilized to detect AKI by offering high sensitivity and selectivity. However, enzyme involvement protocols also increase the system's complexity and cost due to the requirement of optimal conditions for efficient reaction and a well-equiped facility for the production, yet an enzyme-free strategy would be an alternative to eliminate these drawbacks. ${ }^{136}$ Overall, these aforementioned issues along with potential strategies would enable improvement of both current and future diagnostic procedures for AKI diagnosis. Due to their universal nature, these strategies cannot be limited to AKI, and they can be adapted for the other diseases and biosensing strategies as well.

\section{AUTHOR INFORMATION}

\section{Corresponding Author}

Fatih Inci - UNAM-National Nanotechnology Research Center, Bilkent University, 06800 Ankara, Turkey; Institute of Materials Science and Nanotechnology, Bilkent University, 06800 Ankara, Turkey; (o orcid.org/0000-0002-99185038; Email: finci@bilkent.edu.tr

\section{Author \\ Esma Derin - UNAM-National Nanotechnology Research Center, Bilkent University, 06800 Ankara, Turkey; Institute of Materials Science and Nanotechnology, Bilkent University, 06800 Ankara, Turkey}

Complete contact information is available at:

https://pubs.acs.org/10.1021/acssensors.1c01781

\section{Notes}

The authors declare no competing financial interest.

\section{ACKNOWLEDGMENTS}

Dr. Fatih Inci gratefully acknowledges the support from TÜBITAK 2232-International Fellowship for Outstanding Researchers (Project No: 118C254). This publication was produced benefiting from the 2232-International Fellowship for Outstanding Researchers Program of TÜBITAK (Project No: 118C254). However, the entire responsibility of the publication/paper belongs to the owner of the publication/ paper. The financial support received from TÜBİTAK does not mean that the content of the publication is approved in a scientific sense by TÜBITAK. This work was supported by the BAGEP Award of the Science Academy. The authors would like to acknowledge Dr. Ismail Eş for his careful edits and comments on the manuscript.

\section{VOCABULARY}

Kidney, an organ in human body responsible for cleaning blood and transferring waste to urine; Biomarker, a molecule that indicates biological state or condition; Bioreceptor, a molecule that specifically recognizes a target molecule, which is mostly biomarker from a diagnostic perspective; Limit of detection (LOD), the lowest quantity of a concentration of target molecules that can be distinguished reliably from the assay background; Linear dynamic range, the range of concentrations where analytical signals are directly proportional to analyte concentration tested by an analytical method; Specificity, a strong and selective affinity between bioreceptors and target analytes; Label-free detection, a term for the absence of any labels or tags (e.g., enzymes, fluorescent tags, nanoparticles or radioactive molecules) that enhance the signals collected from an analytical measurement, and therefore, provide more direct information between target analytes and bioreceptors that can be acquired from the method

\section{REFERENCES}

(1) Chawla, L. S.; Bellomo, R.; Bihorac, A.; Goldstein, S. L.; Siew, E. D.; Bagshaw, S. M.; Bittleman, D.; Cruz, D.; Endre, Z.; Fitzgerald, R. L.; Forni, L.; Kane-Gill, S. L.; Hoste, E.; Koyner, J.; Liu, K. D.; MacEdo, E.; Mehta, R.; Murray, P.; Nadim, M.; Ostermann, M.; Palevsky, P. M.; Pannu, N.; Rosner, M.; Wald, R.; Zarbock, A.; Ronco, C.; Kellum, J. A. Acute Kidney Disease and Renal Recovery: Consensus Report of the A[1] L. S. Chawla et Al., "Acute Kidney Disease and Renal Recovery: Consensus Report of the Acute Disease Quality Initiative (ADQI) 16 Workgroup,. Nat. Rev. Nephrol. 2017, 13 (4), 241; Nat. Rev. Nephrol. 2017, 13 (4), 241-257.

(2) Deng, B.; Lin, Y.; Chen, Y.; Ma, S.; Cai, Q.; Wang, W.; Li, B.; Liu, T.; Zhou, P.; He, R.; Ding, F. Plasmacytoid Dendritic Cells Promote Acute Kidney Injury by Producing Interferon- $\alpha$. Cell. Mol. Immunol. 2021, 18, 219.

(3) Wang, H. E.; Muntner, P.; Chertow, G. M.; Warnock, D. G. Acute Kidney Injury and Mortality in Hospitalized Patients. Am. J. Nephrol. 2012, 35 (4), 349-355.

(4) International Society of Nephrology. F KDIGO Clinical Practice Guideline for Acute Kidney Injury: Online Appendices A-F; Official Journal of the International Society of Nephrology 2012; Vol. 2, Issue 1. https://kdigo.org/wp-content/uploads/2016/10/KDIGO-AKISuppl-Appendices-A-F_March2012.pdf

(5) Rewa, O.; Bagshāw, S. M. Acute Kidney Injury-Epidemiology, Outcomes and Economics. Nat. Rev. Nephrol. 2014, 10 (4), 193-207.

(6) Ronco, C.; Bellomo, R.; Kellum, J. A. Seminar Acute Kidney Injury. Lancet 2019, 394 (10212), 1949-1964.

(7) Edelstein, C. L. Biomarkers in Acute Kidney Injury. Biomarkers of Kidney Disease 2017, 241.

(8) Cardenas-Gonzalez, M.; Pavkovic, M.; Vaidya, V. S. Biomarkers of Acute Kidney Injury. Compr. Toxicol. Third Ed. 2018, 14-15, 147163.

(9) International Society of Nephrology. KDIGO Clinical Practice Guideline for Acute Kidney Injury. Off. J. Int. Soc. Nephrol. 2012, 2 (1).

(10) Tabata, T.; Sugiyama, N.; Otsuki, Y.; Kondo, Y. Interleukin-24 Is a Novel Diagnostic Biomarker for the Severity of Acute Kidney Injury. Med. Mol. Morphol. 2020, 53, 115.

(11) Murray, P. T.; Mehta, R. L.; Shaw, A.; Ronco, C.; Endre, Z.; Kellum, J. A.; Chawla, L. S.; Cruz, D.; Ince, C.; Okusa, M. D. Potential Use of Biomarkers in Acute Kidney Injury: Report and Summary of Recommendations from the 10th Acute Dialysis Quality Initiative Consensus Conference. Kidney Int. 2014, 85 (3), 513-521.

(12) Ostermann, M.; Joannidis, M. Acute Kidney Injury 2016: Diagnosis and Diagnostic Workup. Crit. Care 2016, 20 (1), 1-13.

(13) Lameire, N. H.; Bagga, A.; Cruz, D.; De Maeseneer, J.; Endre, Z.; Kellum, J. A.; Liu, K. D.; Mehta, R. L.; Pannu, N.; Van Biesen, W.; Vanholder, R. Acute Kidney Injury: An Increasing Global Concern. Lancet 2013, 382 (9887), 170-179.

(14) Bienholz, A.; Wilde, B.; Kribben, A. From the Nephrologist's Point of View: Diversity of Causes and Clinical Features of Acute Kidney Injury. Clin. Kidney J. 2015, 8 (4), 405-414.

(15) Waikar, S. S.; Bonventre, J. V. Biomarkers for the Diagnosis of Acute Kidney Injury. Nephron - Clin. Pract. 2008, 109 (4), c192.

(16) Kellum, J. A.; Prowle, J. R. Paradigms of Acute Kidney Injury. Nat. Rev. Nephrol. 2018, 14 (4), 217-230.

(17) Srisawat, N.; Kellum, J. A. The Role of Biomarkers in Acute Kidney Injury. Crit. Care Clin. 2020, 36 (1), 125-140.

(18) Cherry, A. D.; Hauck, J. N.; Andrew, B. Y.; Li, Y. J.; Privratsky, J. R.; Kartha, L. D.; Nicoara, A.; Thompson, A.; Mathew, J. P.; 
Stafford-Smith, M. Intraoperative Renal Resistive Index Threshold as an Acute Kidney Injury Biomarker. J. Clin. Anesth. 2020, 61, 109626. (19) Jones, T. F.; Bekele, S.; O'Dwyer, M. J.; Prowle, J. R. MicroRNAs in Acute Kidney Injury. Nephron 2018, 140 (2), 124128.

(20) Xu, Y.; Xie, Y.; Shao, X.; Ni, Z.; Mou, S. L-FABP: A Novel Biomarker of Kidney Disease. Clin. Chim. Acta 2015, 445, 85-90.

(21) Vincent, J.-L.; Hall, J. B. Encyclopedia of Intensive Care Medicine; 2012. DOI: $10.1007 / 978-3-642-00418-6$.

(22) Charlton, J. R.; Portilla, D.; Okusa, M. D. A Basic Science View of Acute Kidney Injury Biomarkers. Nephrol., Dial., Transplant. 2014, 29 (7), 1301-1311.

(23) Obermuller, N.; Geiger, H.; Weipert, C.; Urbschat, A. Current Developments in Early Diagnosis of Acute Kidney Injury. Int. Urol. Nephrol. 2014, 46 (46), 1-7.

(24) Buonafine, M.; Martinez-Martinez, E.; Jaisser, F. More than a Simple Biomarker: The Role of NGAL in Cardiovascular and Renal Diseases. Clin. Sci. 2018, 132 (9), 909-923.

(25) Ning, M.; Mao, X.; Niu, Y.; Tang, B.; Shen, H. Usefulness and Limitations of Neutrophil Gelatinase-Associated Lipocalin in the Assessment of Kidney Diseases. J. Lab. Precis. Med. 2018, 3 (3), 1-1.

(26) Ghadrdan, E.; Ebrahimpour, S.; Sadighi, S.; Chaibakhsh, S.; Jahangard-Rafsanjani, Z. Evaluation of Urinary Neutrophil GelatinaseAssociated Lipocalin and Urinary Kidney Injury Molecule-1 as Biomarkers of Renal Function in Cancer Patients Treated with Cisplatin. J. Oncol. Pharm. Pract. 2020, 26, 1643.

(27) Ichimura, T.; Bonventre, J. V.; Bailly, V.; Wei, H.; Hession, C. A.; Cate, R. L.; Sanicola, M. Kidney Injury Molecule-1 (KIM-1), a Putative Epithelial Cell Adhesion Molecule Containing a Novel Immunoglobulin Domain, Is up-Regulated in Renal Cells after Injury. J. Biol. Chem. 1998, 273 (7), 4135-4142.

(28) Song, J.; Yu, J.; Prayogo, G. W.; Cao, W.; Wu, Y.; Jia, Z. Understanding Kidney Injury Molecule $1:$ A Novel Immune Factor in Kidney Pathophysiology. Am. J. Transl. Res. 2019, 11 (3), 1219-1229.

(29) Oh, D. J. A Long Journey for Acute Kidney Injury Biomarkers. Renal Failure 2020, 42 (1), 154-165.

(30) Devarajan, P. Emerging Biomarkers of Acute Kidney Injury. Contrib. Nephrol. 2007, 156, 203-212.

(31) Liu, Y.; Guo, W.; Zhang, J.; Xu, C.; Yu, S.; Mao, Z.; Wu, J.; Ye, C.; Mei, C.; Dai, B. Urinary Interleukin 18 for Detection of Acute Kidney Injury: A Meta-Analysis. Am. J. Kidney Dis. 2013, 62 (6), $1058-1067$.

(32) Dinarello, C. A.; Novick, D.; Kim, S.; Kaplanski, G. Front. Immunol. 2013, 4, 1-10.

(33) Lin, X.; Yuan, J.; Zhao, Y.; Zha, Y. Urine Interleukin-18 in Prediction of Acute Kidney Injury: A Systemic Review and MetaAnalysis. J. Nephrol. 2015, 28 (1), 7-16.

(34) Mussap, M.; Ruzzante, N.; Varagnolo, M.; Plebani, M. Quantitative Automated Particle-Enhanced Immunonephelometric Assay for the Routinary Measurement of Human Cystatin C. Clin. Chem. Lab. Med. 1998, 36 (11), 859-865.

(35) Masson, J. F. Surface Plasmon Resonance Clinical Biosensors for Medical Diagnostics. ACS Sensors 2017, 2 (1), 16-30.

(36) Mousavi, M. Z.; Chen, H. Y.; Lee, K. L.; Lin, H.; Chen, H. H.; Lin, Y. F.; Wong, C. S.; Li, H. F.; Wei, P. K.; Cheng, J. Y. Urinary Micro-RNA Biomarker Detection Using Capped Gold Nanoslit SPR in a Microfluidic Chip. Analyst 2015, 140 (12), 4097-4104.

(37) Ozen, M. O.; Inci, F.; Karaaslan, M. G.; Demirci, U.; Henrich, T.; Ahmed, R. Real-Time Biosensing of Proteins on a DVD Nanoplasmonic Grating. Plasmonics in Biology and Medicine XVI 2019, 22.

(38) Ahmed, R.; Ozen, M. O.; Karaaslan, M. G.; Prator, C. A.; Thanh, C.; Kumar, S.; Torres, L.; Iyer, N.; Munter, S.; Southern, S.; Henrich, T. J.; Inci, F.; Demirci, U. Tunable Fano-Resonant Metasurfaces on a Disposable Plastic-Template for Multimodal and Multiplex Biosensing. Adv. Mater. 2020, 32 (19), 1-11.

(39) Le, T. T.; Adamiak, B.; Benton, D. J.; Johnson, C. J.; Sharma, S.; Fenton, R.; McCauley, J. W.; Iqbal, M.; Cass, A. E. G. Aptamer-
Based Biosensors for the Rapid Visual Detection of Flu Viruses. Chem. Commun. 2014, 50 (98), 15533-15536.

(40) Zhai, L.; Wang, T.; Kang, K.; Zhao, Y.; Shrotriya, P.; NilsenHamilton, M. An RNA Aptamer-Based Microcantilever Sensor to Detect the Inflammatory Marker, Mouse Lipocalin-2. Anal. Chem. 2012, 84 (20), 8763-8770.

(41) Erdem, Ö.; Saylan, Y.; Cihangir, N.; Denizli, A. Molecularly Imprinted Nanoparticles Based Plasmonic Sensors for Real-Time Enterococcus Faecalis Detection. Biosens. Bioelectron. 2019, 126, 608614.

(42) Saylan, Y.; Erdem, Ö.; Inci, F.; Denizli, A. Advances in Biomimetic Systems for Molecular Recognition and Biosensing. Biomimetics 2020, 5 (2), 20.

(43) Bhalla, N.; Jolly, P.; Formisano, N.; Estrela, P. Introduction to Biosensors. Essays Biochem. 2016, 60 (1), 1-8.

(44) Morales, M. A.; Halpern, J. M. Guide to Selecting a Biorecognition Element for Biosensors. Bioconjugate Chem. 2018, 29 (10), 3231-3239.

(45) Moreno-Bondi, M.; Navarro-Villoslada, F.; Benito-Pena, E.; Urraca, J. Molecularly Imprinted Polymers as Selective Recognition Elements in Optical Sensing. Curr. Anal. Chem. 2008, 4 (4), 316-340.

(46) Chen, L.; Wang, X.; Lu, W.; Wu, X.; Li, J. Molecular Imprinting: Perspectives and Applications. Chem. Soc. Rev. 2016, 45 (8), 2137-2211.

(47) Ronkainen, N. J.; Halsall, H. B.; Heineman, W. R. Electrochemical Biosensors. Chem. Soc. Rev. 2010, 39 (5), 17471763.

(48) Cho, I. H.; Kim, D. H.; Park, S. Electrochemical Biosensors: Perspective on Functional Nanomaterials for on-Site Analysis. Biomater. Res. 2020, 24 (1), 1-12.

(49) Barsan, M. M.; Ghica, M. E.; Brett, C. M. A. Electrochemical Sensors and Biosensors Based on Redox Polymer/Carbon Nanotube Modified Electrodes: A Review. Anal. Chim. Acta 2015, 881, 1-23.

(50) Fang, Y.; Wang, E. Electrochemical Biosensors on Platforms of Graphene. Chem. Commun. 2013, 49 (83), 9526-9539.

(51) Lin, D.; Pillai, R. G.; Lee, W. E.; Jemere, A. B. An Impedimetric Biosensor for E. Coli O157:H7 Based on the Use of Self-Assembled Gold Nanoparticles and Protein G. Microchim. Acta 2019, 186 (3), $1-9$.

(52) Huang, J.; Xie, Z.; Xie, Z.; Luo, S.; Xie, L.; Huang, L.; Fan, Q.; Zhang, Y.; Wang, S.; Zeng, T. Silver Nanoparticles Coated Graphene Electrochemical Sensor for the Ultrasensitive Analysis of Avian Influenza Virus H7. Anal. Chim. Acta 2016, 913, 121-127.

(53) Tancharoen, C.; Sukjee, W.; Thepparit, C.; Jaimipuk, T.; Auewarakul, P.; Thitithanyanont, A.; Sangma, C. Electrochemical Biosensor Based on Surface Imprinting for Zika Virus Detection in Serum. ACS Sensors 2019, 4 (1), 69-75.

(54) Viswanathan, S.; Radecka, H.; Radecki, J. Electrochemical Biosensors for Food Analysis. Monatsh. Chem. 2009, 140 (8), 891899.

(55) Badihi-Mossberg, M.; Buchner, V.; Rishpon, J. Electrochemical Biosensors for Pollutants in the Environment. Electroanalysis 2007, 19 (19-20), 2015-2028.

(56) Cho, C. H.; Kim, J. H.; Song, D. K.; Park, T. J.; Park, J. P. An Affinity Peptide-Incorporated Electrochemical Biosensor for the Detection of Neutrophil Gelatinase-Associated Lipocalin. Biosens. Bioelectron. 2019, 142 (May), 111482.

(57) Diouf, A.; Motia, S.; El Alami El Hassani, N.; El Bari, N.; Bouchikhi, B. Development and Characterization of an Electrochemical Biosensor for Creatinine Detection in Human Urine Based on Functional Molecularly Imprinted Polymer. J. Electroanal. Chem. 2017, 788, 44-53.

(58) Dasgupta, P.; Kumar, V.; Krishnaswamy, P. R.; Bhat, N. Serum Creatinine Electrochemical Biosensor on Printed Electrodes Using Monoenzymatic Pathway to 1-methylhydantoin Detection. ACS Omega 2020, 5 (35), 22459-22464.

(59) Li, H.; Mu, Y.; Yan, J.; Cui, D.; Ou, W.; Wan, Y.; Liu, S. LabelFree Photoelectrochemical Immunosensor for Neutrophil Gelatinase- 
Associated Lipocalin Based on the Use of Nanobodies. Anal. Chem. 2015, 87 (3), 2007-2015.

(60) Kannan, P.; Tiong, H. Y.; Kim, D. H. Highly Sensitive Electrochemical Determination of Neutrophil Gelatinase-Associated Lipocalin for Acute Kidney Injury. Biosens. Bioelectron. 2012, 31 (1), $32-36$.

(61) Yukird, J.; Wongtangprasert, T.; Rangkupan, R.; Chailapakul, O.; Pisitkun, T.; Rodthongkum, N. Label-Free Immunosensor Based on Graphene/Polyaniline Nanocomposite for Neutrophil GelatinaseAssociated Lipocalin Detection. Biosens. Bioelectron. 2017, 87, 249255.

(62) Neves, M. M. P. S.; Nouws, H. P. A.; Santos-Silva, A.; DelerueMatos, C. Neutrophil Gelatinase-Associated Lipocalin Detection Using a Sensitive Electrochemical Immunosensing Approach. Sens. Actuators, B 2020, 304, 127285.

(63) Trindade, E. K. G.; Silva, B. V. M.; Dutra, R. F. A Probeless and Label-Free Electrochemical Immunosensor for Cystatin C Detection Based on Ferrocene Functionalized-Graphene Platform. Biosens. Bioelectron. 2019, 138 (May), 111311.

(64) Lopes, P.; Costa-Rama, E.; Beirão, I.; Nouws, H. P. A.; SantosSilva, A.; Delerue-Matos, C. Disposable Electrochemical Immunosensor for Analysis of Cystatin C, a CKD Biomarker. Talanta 2019, 201 (April), 211-216.

(65) Desai, D.; Kumar, A.; Bose, D.; Datta, M. Ultrasensitive Sensor for Detection of Early Stage Chronic Kidney Disease in Human. Biosens. Bioelectron. 2018, 105, 90-94.

(66) Gomes, R. S.; Gomez-Rodríguez, B. A.; Fernandes, R.; Sales, M. G. F.; Moreira, F. T. C.; Dutra, R. F. Plastic Antibody of Polypyrrole/ Multiwall Carbon Nanotubes on Screen-Printed Electrodes for Cystatin C Detection. Biosensors 2021, 11 (6), 175.

(67) Barizuddin, S.; Bok, S.; Gangopadhyay, S. Plasmonic Sensors for Disease Detection - A Review. J. Nanomed. Nanotechnol. 2016, 7 (3), 1 DOI: $10.4172 / 2157-7439.1000373$.

(68) Wang, Q.; Jing, J. Y.; Wang, B. T. Highly Sensitive SPR Biosensor Based on Graphene Oxide and Staphylococcal Protein A Co-Modified TFBG for Human IgG Detection. IEEE Trans. Instrum. Meas. 2019, 68 (9), 3350-3357.

(69) Jiang, W. S.; Xin, W.; Xun, S.; Chen, S. N.; Gao, X. G.; Liu, Z. B.; Tian, J. G. Reduced Graphene Oxide-Based Optical Sensor for Detecting Specific Protein. Sens. Actuators, B 2017, 249, 142-148.

(70) Liu, J.; Jalali, M.; Mahshid, S.; Wachsmann-Hogiu, S. Are Plasmonic Optical Biosensors Ready for Use in Point-of-Need Applications? Analyst 2020, 145 (2), 364-384.

(71) Inci, F.; Celik, U.; Turken, B.; Özer, H. Ö.; Kok, F. N. Construction of P-Glycoprotein Incorporated Tethered Lipid Bilayer Membranes. Biochem. Biophys. Reports 2015, 2, 115-122.

(72) Tokel, O.; Inci, F.; Demirci, U. Advances in Plasmonic Technologies for Point of Care Applications. Chem. Rev. 2014, 114 (11), 5728-5752.

(73) Wang, J.; Luo, Y.; Zhang, B.; Chen, M.; Huang, J.; Zhang, K.; Gao, W.; Fu, W.; Jiang, T.; Liao, P. Rapid Label-Free Identification of Mixed Bacterial Infections by Surface Plasmon Resonance. J. Transl. Med. 2011, 9, 1-9.

(74) Inci, F.; Saylan, Y.; Kojouri, A. M.; Ogut, M. G.; Denizli, A.; Demirci, U. A Disposable Microfluidic-Integrated Hand-Held Plasmonic Platform for Protein Detection. Appl. Mater. Today 2020, $18,14-16$.

(75) Saylan, Y.; Denizli, A. Molecular Fingerprints of Hemoglobin on a Nanofilm Chip. Sensors 2018, 18 (9), 3016.

(76) Moore, T. J.; Moody, A. S.; Payne, T. D.; Sarabia, G. M.; Daniel, A. R.; Sharma, B. In Vitro and in Vivo Sers Biosensing for Disease Diagnosis. Biosensors 2018, 8 (2), 46.

(77) Inci, F.; Tokel, O.; Wang, S.; Gurkan, U. A.; Tasoglu, S.; Kuritzkes, D. R.; Demirci, U. Nanoplasmonic Quantitative Detection of Intact Viruses from Unprocessed Whole Blood. ACS Nano 2013, 7 (6), 4733-4745.

(78) Inci, F.; Karaaslan, M. G.; Mataji-Kojouri, A.; Shah, P. A.; Saylan, Y.; Zeng, Y.; Avadhani, A.; Sinclair, R.; Lau, D. T. Y.; Demirci, U. Enhancing the Nanoplasmonic Signal by a Nanoparticle
Sandwiching Strategy to Detect Viruses. Appl. Mater. Today 2020, 20, 100709 .

(79) Mataji-Kojouri, A.; Ozen, M. O.; Shahabadi, M.; Inci, F.; Demirci, U. Entangled Nanoplasmonic Cavities for Estimating Thickness of Surface-Adsorbed Layers. ACS Nano 2020, 14, 8518.

(80) Tokel, O.; Yildiz, U. H.; Inci, F.; Durmus, N. G.; Ekiz, O. O.; Turker, B.; Cetin, C.; Rao, S.; Sridhar, K.; Natarajan, N.; Shafiee, H.; Dana, A.; Demirci, U. Portable Microfluidic Integrated Plasmonic Platform for Pathogen Detection. Sci. Rep. 2015, 5, 1-9.

(81) Inci, F.; Filippini, C.; Baday, M.; Ozen, M. O.; Calamak, S.; Durmus, N. G.; Wang, S. Q.; Hanhauser, E.; Hobbs, K. S.; Juillard, F.; Kuang, P. P.; Vetter, M. L.; Carocci, M.; Yamamoto, H. S.; Takagi, Y.; Yildiz, U. H.; Akin, D.; Wesemann, D. R.; Singhal, A.; Yang, P. L.; Nibert, M. L.; Fichorova, R. N.; Lau, D. T. Y.; Henrich, T. J.; Kaye, K. M.; Schachter, S. C.; Kuritzkes, D. R.; Steinmetz, L. M.; Gambhir, S. S.; Davis, R. W.; Demirci, U. Multitarget, Quantitative Nanoplasmonic Electrical Field-Enhanced Resonating Device (NE2RD) for Diagnostics. Proc. Natl. Acad. Sci. U. S. A. 2015, 112 (32), E4354E4363.

(82) Luan, J.; Morrissey, J. J.; Wang, Z.; Derami, H. G.; Liu, K. K.; Cao, S.; Jiang, Q.; Wang, C.; Kharasch, E. D.; Naik, R. R.; Singamaneni, S. Add-on Plasmonic Patch as a Universal Fluorescence Enhancer. Light: Sci. Appl. 2018, 7 (1), 1 DOI: 10.1038/s41377-0180027-8.

(83) Zeidan, E.; Li, S.; Zhou, Z.; Miller, J.; Sandros, M. G. SingleMultiplex Detection of Organ Injury Biomarkers Using SPRi Based Nano-Immunosensor. Sci. Rep. 2016, 6, 2-9.

(84) Luan, J.; Xu, T.; Cashin, J.; Morrissey, J. J.; Kharasch, E. D.; Singamaneni, S. Environmental Stability of Plasmonic Biosensors Based on Natural versus Artificial Antibody. Anal. Chem. 2018, 90 (13), 7880-7887.

(85) Tian, L.; Liu, K. K.; Morrissey, J. J.; Gandra, N.; Kharasch, E. D.; Singamaneni, S. Gold Nanocages with Built-in Artificial Antibodies for Label-Free Plasmonic Biosensing. J. Mater. Chem. B 2014, 2 (2), 167-170.

(86) Liu, J. T.; Lin, P. S.; Hsin, Y. M.; Tsai, J. Z.; Chen, W. Y. Surface Plasmon Resonance Biosensor for Microalbumin Detection. J. Taiwan Inst. Chem. Eng. 2011, 42 (5), 696-700.

(87) Vijayan, A.; Faubel, S.; Askenazi, D. J.; Cerda, J.; Fissell, W. H.; Heung, M.; Humphreys, B. D.; Koyner, J. L.; Liu, K. D.; Mour, G.; Nolin, T. D.; Bihorac, A.; Basu, R. K.; Bhatt, U. Y.; Connor, M. J.; Davidson, A. J.; De Caestecker, M. P.; Doi, K.; Golestaneh, L.; Mour, G. K.; Singh, P.; Thakar, C. V. Clinical Use of the Urine Biomarker [TIMP-2] $\times$ [IGFBP7] for Acute Kidney Injury Risk Assessment. Am. J. Kidney Dis. 2016, 68 (1), 19-28.

(88) Jang, D. H.; Choi, Y.; Choi, Y. S.; Kim, S. M.; Kwak, H.; Shin, S. H.; Hong, S. Sensitive and Selective Analysis of a Wide Concentration Range of IGFBP7 Using a Surface Plasmon Resonance Biosensor. Colloids Surf., B 2014, 123, 887-891.

(89) Hassanain, W. A.; Izake, E. L.; Ayoko, G. A. Spectroelectrochemical Nanosensor for the Determination of Cystatin $\mathrm{C}$ in Human Blood. Anal. Chem. 2018, 90 (18), 10843-10850.

(90) Erdem, Ö.; Derin, E.; Sagdic, K.; Yilmaz, E. G.; Inci, F. Smart Materials-Integrated Sensor Technologies for COVID-19 Diagnosis. Emergent Mater. 2021, 4, 169.

(91) Nath, S.; Pigula, M.; Khan, A. P.; Hanna, W.; Ruhi, M. K.; Dehkordy, F. M.; Pushpavanam, K.; Rege, K.; Moore, K.; Tsujita, Y.; Conrad, C.; Inci, F.; del Carmen, M. G.; Franco, W.; Celli, J. P.; Demirci, U.; Hasan, T.; Huang, H.-C.; Rizvi, I. Flow-Induced Shear Stress Confers Resistance to Carboplatin in an Adherent ThreeDimensional Model for Ovarian Cancer: A Role for EGFR-Targeted Photoimmunotherapy Informed by Physical Stress. J. Clin. Med. 2020, 9 (4), 924.

(92) Inci, F.; Ozen, M. O.; Saylan, Y.; Miansari, M.; Cimen, D.; Dhara, R.; Chinnasamy, T.; Yuksekkaya, M.; Filippini, C.; Kumar, D. K.; Calamak, S.; Yesil, Y.; Durmus, N. G.; Duncan, G.; Klevan, L.; Demirci, U. A Novel On-Chip Method for Differential Extraction of Sperm in Forensic Cases. Adv. Sci. 2018, 5 (9), 1800121. 
(93) Deshmukh, S.; Inci, F.; Karaaslan, M. G.; Ogut, M. G.; Duncan, D.; Klevan, L.; Duncan, G.; Demirci, U. A Confirmatory Test for Sperm in Sexual Assault Samples Using a Microfluidic-Integrated Cell Phone Imaging System. Forensic Sci. Int.: Genet. 2020, 48 (May), 102313.

(94) Inci, F.; Karaaslan, M. G.; Gupta, R.; Avadhani, A.; Ogut, M. G.; Atila, E. E.; Duncan, G.; Klevan, L.; Demirci, U. Bio-Inspired Magnetic Beads for Isolation of Sperm From Heterogenous Samples in Forensic Applications. Forensic Sci. Int.: Genet. 2021, 52, 102451.

(95) Inan, H.; Wang, S.; Inci, F.; Baday, M.; Zangar, R.; Kesiraju, S.; Anderson, K. S.; Cunningham, B. T.; Demirci, U. Isolation, Detection, and Quantification of Cancer Biomarkers in HPV-Associated Malignancies. Sci. Rep. 2017, 7 (1), 1-11.

(96) Liang, L. G.; Kong, M. Q.; Zhou, S.; Sheng, Y. F.; Wang, P.; Yu, T.; Inci, F.; Kuo, W. P.; Li, L. J.; Demirci, U.; Wang, S. Q. An Integrated Double-Filtration Microfluidic Device for Isolation, Enrichment and Quantification of Urinary Extracellular Vesicles for Detection of Bladder Cancer. Sci. Rep. 2017, 7, 1-10.

(97) Erdem, Ö.; Eş, I.; Akceoglu, G. A.; Saylan, Y.; Inci, F. Recent Advances in Microneedle-Based Sensors for Sampling, Diagnosis and Monitoring of Chronic Diseases. Biosensors 2021, 11 (9), 296.

(98) Hong, G.; Diao, S.; Antaris, A. L.; Dai, H. Carbon Nanomaterials for Biological Imaging and Nanomedicinal Therapy. Chem. Rev. 2015, 115 (19), 10816-10906.

(99) Akceoglu, G. A.; Saylan, Y.; Inci, F. A Snapshot of Microfluidics in Point-of-Care Diagnostics: Multifaceted Integrity with Materials and Sensors. Adv. Mater. Technol. 2021, 6, 2100049.

(100) Mani, V.; Paleja, B.; Larbi, K.; Kumar, P.; Tay, J. A.; Siew, J. Y.; Inci, F.; Wang, S. Q.; Chee, C.; Wang, Y. T.; Demirci, U.; De Libero, G.; Singhal, A. Microchip-Based Ultrafast Serodiagnostic Assay for Tuberculosis. Sci. Rep. 2016, 6 (May), 1-11.

(101) Shirejini, S. Z.; Inci, F. The Yin and Yang of Exosome Isolation Methods: Conventional Practice, Microfluidics, and Commercial Kits. Biotechnol. Adv. 2021, 107814.

(102) Asghar, W.; Yuksekkaya, M.; Shafiee, H.; Zhang, M.; Ozen, M. O.; Inci, F.; Kocakulak, M.; Demirci, U. Engineering Long Shelf Life Multi-Layer Biologically Active Surfaces on Microfluidic Devices for Point of Care Applications. Sci. Rep. 2016, 6, 1-10.

(103) Martin-Gracia, B.; Martin-Barreiro, A.; Cuestas-Ayllon, C.; Grazu, V.; Line, A.; Llorente, A.; M. de la Fuente, J.; Moros, M. Nanoparticle-Based Biosensors for Detection of Extracellular Vesicles in Liquid Biopsies. J. Mater. Chem. B 2020, 8 (31), 6710-6738.

(104) Kim, J. E.; Choi, J. H.; Colas, M.; Kim, D. H.; Lee, H. GoldBased Hybrid Nanomaterials for Biosensing and Molecular Diagnostic Applications. Biosens. Bioelectron. 2016, 80, 543-559.

(105) Chung, H. J.; Pellegrini, K. L.; Chung, J.; Wanigasuriya, K.; Jayawardene, I.; Lee, K.; Lee, H.; Vaidya, V. S.; Weissleder, R. Nanoparticle Detection of Urinary Markers for Point-of-Care Diagnosis of Kidney Injury. PLoS One 2015, 10 (7), 1-11.

(106) Tao, J.; Zhao, P.; Zeng, Q. The Determination of Cystatin C in Serum Based on Label-Free and near-Infrared Light Emitted PbS@ BSA QDs. J. Mater. Chem. B 2016, 4 (24), 4258-4262.

(107) Banerjee, R.; Jaiswal, A. Recent Advances in NanoparticleBased Lateral Flow Immunoassay as a Point-of-Care Diagnostic Tool for Infectious Agents and Diseases. Analyst 2018, 143 (9), 19701996

(108) De La Rica, R.; Stevens, M. M. Plasmonic ELISA for the Ultrasensitive Detection of Disease Biomarkers with the Naked Eye. Nat. Nanotechnol. 2012, 7 (12), 821-824.

(109) Liu, D.; Wang, Z.; Jiang, X. Gold Nanoparticles for the Colorimetric and Fluorescent Detection of Ions and Small Organic Molecules. Nanoscale 2011, 3 (4), 1421-1433.

(110) Luo, X.; Morrin, A.; Killard, A. J.; Smyth, M. R. Application of Nanoparticles in Electrochemical Sensors and Biosensors. Electroanalysis 2006, 18 (4), 319-326.

(111) Tian, F.; Bonnier, F.; Casey, A.; Shanahan, A. E.; Byrne, H. J. Surface Enhanced Raman Scattering with Gold Nanoparticles: Effect of Particle Shape. Anal. Methods 2014, 6 (22), 9116-9123.
(112) Inci, F.; Karaaslan, M. G.; Mataji-Kojouri, A.; Shah, P. A.; Saylan, Y.; Zeng, Y.; Avadhani, A.; Sinclair, R.; Lau, D. T.-Y.; Demirci, U. Enhancing the Nanoplasmonic Signal by a Nanoparticle Sandwiching Strategy to Detect Viruses. Appl. Mater. Today 2020, 20, 100709 .

(113) Ly, T. N.; Park, S.; Park, S. J. Detection of HIV-1 Antigen by Quartz Crystal Microbalance Using Gold Nanoparticles. Sens. Actuators, B 2016, 237, 452-458.

(114) Issadore, D.; Min, C.; Liong, M.; Chung, J.; Weissleder, R.; Lee, H. Miniature Magnetic Resonance System for Point-of-Care Diagnostics. Lab Chip 2011, 11 (13), 2282-2287.

(115) Zhang, J. X. J.; Hoshino, K. Microfluidics and Micro Total Analytical Systems. In Molecular Sensors and Nanodevices 2019, 113179.

(116) Ren, K.; Zhou, J.; Wu, H. Materials for Microfluidic Chip Fabrication. Acc. Chem. Res. 2013, 46 (11), 2396-2406.

(117) Zhu, H.; Fohlerová, Z.; Pekárek, J.; Basova, E.; Neužil, P. Recent Advances in Lab-on-a-Chip Technologies for Viral Diagnosis. Biosens. Bioelectron. 2020, 153, 112041.

(118) Whitesides, G. M. The Origins and the Future of Microfluidics. Nature 2006, 442 (7101), 368-373.

(119) Damhorst, G. L.; Murtagh, M.; Rodriguez, W. R.; Bashir, R. Microfluidics and Nanotechnology for Detection of Global Infectious Diseases. Proc. IEEE 2015, 103 (2), 150-160.

(120) Huynh, T.; Sun, B.; Li, L.; Nichols, K. P.; Koyner, J. L.; Ismagilov, R. F. Chemical Analog-to-Digital Signal Conversion Based on Robust Threshold Chemistry and Its Evaluation in the Context of Microfluidics-Based Quantitative Assays. J. Am. Chem. Soc. 2013, 135 (39), 14775-14783.

(121) Pysz, M. A.; Gambhir, S. S.; Willmann, J. K. Molecular Imaging: Current Status and Emerging Strategies. Clin. Radiol. 2010, 65 (7), 500-516.

(122) Hickson, J. In Vivo Optical Imaging: Preclinical Applications and Considerations. Urol. Oncol. Semin. Orig. Investig. 2009, 27 (3), 295-297.

(123) An, R.; Wei, S.; Huang, Z.; Liu, F.; Ye, D. An Activatable Chemiluminescent Probe for Sensitive Detection of $\gamma$-Glutamyl Transpeptidase Activity in Vivo. Anal. Chem. 2019, 91 (21), 13639-13646.

(124) Schäferling, M.; Grögel, D. B. M.; Schreml, S. Luminescent Probes for Detection and Imaging of Hydrogen Peroxide. Microchim. Acta 2011, 174 (1), 1-18.

(125) Texier, I.; Josserand, V.; Garanger, E.; Razkin, J.; Jin, Z.; Dumy, P.; Favrot, M.; Boturyn, D.; Coll, J.-L. Luminescent Probes for Optical in Vivo Imaging. Proc. SPIE 2005, 5704, 16.

(126) Yan, F.; Tian, X.; Luan, Z.; Feng, L.; Ma, X.; James, T. D. NAG-Targeting Fluorescence Based Probe for Precision Diagnosis of Kidney Injury. Chem. Commun. 2019, 55 (13), 1955-1958.

(127) Tang, Z.; Song, B.; Zhang, W.; Guo, L.; Yuan, J. Precise Monitoring of Drug-Induced Kidney Injury Using an Endoplasmic Reticulum-Targetable Ratiometric Time-Gated Luminescence Probe for Superoxide Anions. Anal. Chem. 2019, 91 (21), 14019-14028.

(128) Huang, J.; Lyu, Y.; Li, J.; Cheng, P.; Jiang, Y.; Pu, K. A RenalClearable Duplex Optical Reporter for Real-Time Imaging of Contrast-Induced Acute Kidney Injury. Angew. Chem. 2019, 131 (49), 17960-17968.

(129) Cheng, P.; Miao, Q.; Huang, J.; Li, J.; Pu, K. Multiplex Optical Urinalysis for Early Detection of Drug-Induced Kidney Injury. Anal. Chem. 2020, 92, 6166.

(130) Lisowska-Myjak, B. Serum and Urinary Biomarkers of Acute Kidney Injury. Blood Purif. 2010, 29 (4), 357-365.

(131) Quesada, A.; Vargas, F.; Montoro-Molina, S.; O’Valle, F.; Rodríguez-Martínez, M. D.; Osuna, A.; Prieto, I.; Ramírez, M.; Wangensteen, R. Urinary Aminopeptidase Activities as Early and Predictive Biomarkers of Renal Dysfunction in Cisplatin-Treated Rats. PLoS One 2012, 7, 1-9.

(132) Huang, J.; Li, J.; Lyu, Y.; Miao, Q.; Pu, K. Molecular Optical Imaging Probes for Early Diagnosis of Drug-Induced Acute Kidney Injury. Nat. Mater. 2019, 18 (10), 1133-1143. 
(133) Huang, J.; Huang, J.; Cheng, P.; Jiang, Y.; Pu, K. Near-Infrared Chemiluminescent Reporters for In Vivo Imaging of Reactive Oxygen and Nitrogen Species in Kidneys. Adv. Funct. Mater. 2020, 30 (39), 2003628.

(134) Wang, F.; Jiang, X.; Xiang, H.; Wang, N.; Zhang, Y.; Yao, X.; Wang, P.; Pan, H.; Yu, L.; Cheng, Y.; Hu, Y.; Lin, W.; Li, X. An Inherently Kidney-Targeting near-Infrared Fluorophore Based Probe for Early Detection of Acute Kidney Injury. Biosens. Bioelectron. 2021, $172,112756$.

(135) Liu, L.; Jiang, L.; Yuan, W.; Liu, Z.; Liu, D.; Wei, P.; Zhang, X.; Yi, T. Dual-Modality Detection of Early-Stage Drug-Induced Acute Kidney Injury by an Activatable Probe. ACS Sensors 2020, 5 (8), 2457-2466.

(136) Li, S. K.; Liu, Z. T.; Li, J. Y.; Chen, A. Y.; Chai, Y. Q.; Yuan, R.; Zhuo, Y. Enzyme-Free Target Recycling and Double-Output Amplification System for Electrochemiluminescent Assay of Mucin 1 with MoS2 Nanoflowers as Co-Reaction Accelerator. ACS Appl. Mater. Interfaces 2018, 10 (17), 14483-14490.

(137) Xu, H.; Wang, L.; Ye, H.; Yu, L.; Zhu, X.; Lin, Z.; Wu, G.; Li, X.; Liu, X.; Chen, G. An Ultrasensitive Electrochemical Impedance Sensor for a Special BRCA1 Breast Cancer Gene Sequence Based on Lambda Exonuclease Assisted Target Recycling Amplification. Chem. Commun. 2012, 48 (51), 6390-6392.

(138) Wen, Z.-B.; Liang, W.-B.; Zhuo, Y.; Xiong, C.-Y.; Zheng, Y.N.; Yuan, R.; Chai, Y.-Q. An Efficient Target-Intermediate Recycling Amplification Strategy for Ultrasensitive Fluorescence Assay of Intracellular Lead Ions. Chem. Commun. 2017, 53 (54), 7525-7528.

(139) Wen, S.; Su, Y.; Dai, C.; Jia, J.; Fan, G.; Jiang, L.; Song, R.; Zhu, J. Plasmon Coupling-Enhanced Raman Sensing Platform Integrated with Exonuclease-Assisted Target Recycling Ampli Fi Cation for Ultrasensitive and Selective Detection of MicroRNA-21. Anal. Chem. 2019, 91, 12298.

(140) Hu, Y.; Yu, X.; Zhang, Y.; Zhang, R.; Bai, X.; Lu, M.; Li, J.; Gu, L.; Liu, J.-H.; Yu, B.-Y.; Tian, J. Rapid and Sensitive Detection of NGAL for the Prediction of Acute Kidney Injury via a Polydopamine Nanosphere/Aptamer Nanocomplex Coupled with DNase I-Assisted Recycling Amplification. Analyst 2020, 145, 3620-3625.

(141) Yu, X. A.; Hu, Y.; Zhang, Y.; Zhang, R.; Bai, X.; Gu, L.; Gao, H.; Li, R.; Tian, J.; Yu, B. Y. Integrating the Polydopamine Nanosphere/Aptamers Nanoplatform with a DNase-I-Assisted Recycling Amplification Strategy for Simultaneous Detection of MMP-9 and MMP-2 during Renal Interstitial Fibrosis. ACS Sensors 2020, 5 (4), 1119-1125.

(142) Hu, Y.; Yu, X. A.; Zhang, Y.; Zhang, R.; Bai, X.; Lu, M.; Li, J.; Gu, L.; Liu, J. H.; Yu, B. Y.; Tian, J. Rapid and Sensitive Detection of NGAL for the Prediction of Acute Kidney Injury: Via a Polydopamine Nanosphere/Aptamer Nanocomplex Coupled with DNase I-Assisted Recycling Amplification. Analyst 2020, 145 (10), 3620-3625.

(143) Guzzi, F.; Cirillo, L.; Roperto, R. M.; Romagnani, P.; Lazzeri, E. Molecular Mechanisms of the Acute Kidney Injury to Chronic Kidney Disease Transition: An Updated View. Int. J. Mol. Sci. 2019, 20 (19), 4941.

(144) Gao, H.; Li, J.; Jia, Y.; Yu, X. A.; Qi, J.; Tian, J.; Yu, B. Y. A Hairpin DNA-Fueled Nanoflare for Simultaneous Illumination of Two MicroRNAs in Drug-Induced Nephrotoxic Cells with Target Catalytic Recycling Amplification. Analyst 2019, 144 (24), 71787184.

(145) Huang, X.; Li, J.; Lu, M.; Zhang, W.; Xu, Z.; Yu, B. Y.; Tian, J. Point-of-Care Testing of MicroRNA Based on Personal Glucose Meter and Dual Signal Amplification to Evaluate Drug-Induced Kidney Injury. Anal. Chim. Acta 2020, 1112, 72-79.

(146) Sato, E.; Kamijo-Ikemori, A.; Oikawa, T.; Okuda, A.; Sugaya, T.; Kimura, K.; Nakamura, T.; Shibagaki, Y. Urinary Excretion of Liver-Type Fatty Acid-Binding Protein Reflects the Severity of Sepsis. Ren. Replace. Ther. 2017, 3 (1), 1-10.

(147) Fan, W.; Ankawi, G.; Zhang, J.; Digvijay, K.; Giavarina, D.; Yin, Y.; Ronco, C. Current Understanding and Future Directions in the Application of TIMP-2 and IGFBP7 in AKI Clinical Practice. Clin. Chem. Lab. Med. 2019, 57 (5), 567-576.
(148) Assays for KIM-1 Assays for KIM-1 Acute Kidney Injury Biomarker; https://bioassayworks.com/wp-content/uploads/2017/ 05/ML $0021 \quad 12$ KIM1 Sellsheet 052017 web.pdf.

(149) Bonventre, J. V.; Yang, $\bar{L}$. Cellular Pathophysiology of Ischemic Acute Kidney Injury Find the Latest Version: Science in Medicine Cellular Pathophysiology of Ischemic Acute Kidney Injury. J. Clin. Invest. 2011, 121 (11), 4210-4221.

(150) Gao, L.; Zhong, X.; Jin, J.; Li, J.; Meng, X. Potential Targeted Therapy and Diagnosis Based on Novel Insight into Growth Factors, Receptors, and Downstream Effectors in Acute Kidney Injury and Acute Kidney Injury-Chronic Kidney Disease Progression. Signal Transduct. Target. Ther. 2020, 5, 1-11.

(151) Chawla, L. S.; Eggers, P. W.; Star, R. A.; Kimmel, P. L. Acute Kidney Injury and Chronic Kidney Disease as Interconnected Syndromes. N. Engl. J. Med. 2014, 371 (1), 58-66.

(152) Park, J.; Lin, H. Y.; Assaker, J. P.; Jeong, S.; Huang, C. H.; Kurdi, A.; Lee, K.; Fraser, K.; Min, C.; Eskandari, S.; Routray, S.; Tannous, B.; Abdi, R.; Riella, L.; Chandraker, A.; Castro, C. M.; Weissleder, R.; Lee, H.; Azzi, J. R. Integrated Kidney Exosome Analysis for the Detection of Kidney Transplant Rejection. ACS Nano 2017, 11 (11), 11041-11046.

(153) Bongiovanni, C.; Magrini, L.; Salerno, G.; Gori, C. S.; Cardelli, P.; Hur, M.; Buggi, M.; Di Somma, S. Serum Cystatin C for the Diagnosis of Acute Kidney Injury in Patients Admitted in the Emergency Department. Dis. Markers 2015, 2015, 1.

(154) CMIC HOLDINGS Co. Ltd. Renischem L-FABP Poc Kit; https://www.fabp.jp/eng/wp/wp-content/uploads/2018/06/ 180605 RENISCHEM pocKit.pdf.

(155) Suzuki, G.; Ichibayashi, R.; Yamamoto, S.; Nakamichi, Y.; Watanabe, M.; Honda, M. Clinical Significance of Urinary L-FABP in the Emergency Department. Int. J. Emerg. Med. 2019, 12 (1), 1 DOI: 10.1186/s12245-019-0244-9.

(156) FDA. Evaluation of Automatic Class III Designation for Nephrocheck Test System; 2014; https://www.accessdata.fda.gov/ cdrh_docs/reviews/DEN130031.pdf.

$(15 \overline{7})$ Astute Medical. NEPHROCHECK® Test Kit Package Insert; https://www.astutemedical.com/content/documents/us/ NephroCheck_Test_Package_Insert_US_IVD_(PN_300152)_ RevE.pdf.

(158) Guzzi, L. M.; Bergler, T.; Binnall, B.; Engelman, D. T.; Forni, L.; Germain, M. J.; Gluck, E.; Göcze, I.; Joannidis, M.; Koyner, J. L.; Reddy, V. S.; Rimmelé, T.; Ronco, C.; Textoris, J.; Zarbock, A.; Kellum, J. A. Clinical Use of [TIMP-2] •[IGFBP7] Biomarker Testing to Assess Risk of Acute Kidney Injury in Critical Care: Guidance from an Expert Panel. Crit. Care 2019, 23 (1), 1-9.

(159) Vaidya, V. S.; Ozer, J. S.; Dieterle, F.; Collings, F. B.; Ramirez, V.; Troth, S.; Muniappa, N.; Thudium, D.; Gerhold, D.; Holder, D. J.; Bobadilla, N. A.; Marrer, E.; Perentes, E.; Cordier, A.; Vonderscher, J.; Maurer, G.; Goering, P. L.; Sistare, F. D.; Bonventre, J. V. Kidney Injury Molecule-1 Outperforms Traditional Biomarkers of Kidney Injury in Preclinical Biomarker Qualification Studies. Nat. Biotechnol. 2010, 28 (5), 478-485.

(160) Sabbisetti, V. S.; Ito, K.; Wang, C.; Yang, L.; Mefferd, S. C.; Bonventre, J. V. Novel Assays for Detection of Urinary KIM-1 in Mouse Models of Kidney Injury. Toxicol. Sci. 2013, 131 (1), 13-25.

(161) Coca, S. G.; Singanamala, S.; Parikh, C. R. Chronic Kidney Disease after Acute Kidney Injury: A Systematic Review and MetaAnalysis. Kidney Int. 2012, 81 (5), 442-448.

(162) Coca, S. G. Acute Kidney Injury in Elderly Persons. Am. J. Kidney Dis. 2010, 56 (1), 122-131.

(163) Zhao, H.; Liang, L.; Pan, S.; Liu, Z.; Liang, Y.; Qiao, Y.; Liu, D.; Liu, Z. Diabetes Mellitus as a Risk Factor for Progression from Acute Kidney Injury to Acute Kidney Disease: A Specific Prediction Model. Diabetes, Metab. Syndr. Obes.: Targets Ther. 2021, 14, 23672379.

(164) Bihorac, A.; Yavas, S.; Subbiah, S.; Hobson, C. E. Long-Term Risk of Mortality and Acute Kidney Injury During. Ann. Surg. 2009, 249 (5), 851-858. 
(165) Gomez, H.; Kellum, J. A. Sepsis-Induced Acute Kidney Injury. Curr. Opin. Crit. Care 2016, 22 (6), 546-553.

(166) Finlay, S.; Bray, B.; Lewington, A. J.; Hunter-Rowe, C. T.; Banerjee, A.; Atkinson, J. M.; Jones, M. C. Identification of Risk Factors Associated with Acute Kidney Injury in Patients Admitted to Acute Medical Units. Clin. Med. J. R. Coll. Physicians London 2013, 13 (3), 233-238.

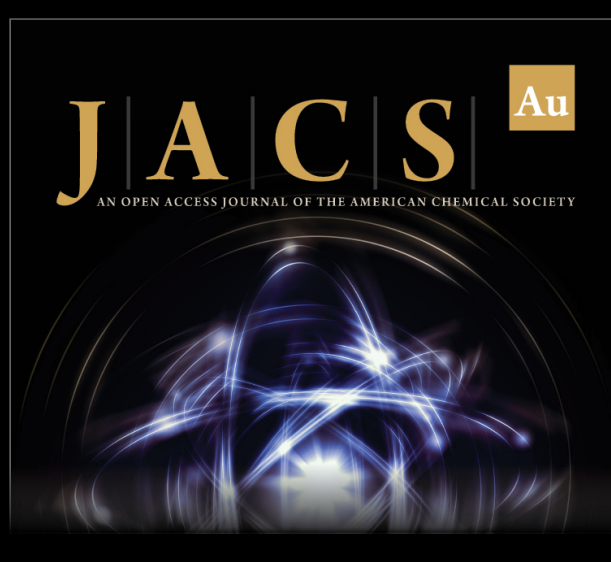

Editor-in-Chief

Prof. Christopher $\mathbf{W}$. Jones

Georgia Institute of Technology, USA

Open for Submissions 